\title{
Biomolecular Assembly of Gold Nanocrystals
}

\author{
by
}

Christine Marya Micheel

\author{
A.B. (Washington University) 1999 \\ A dissertation submitted in partial satisfaction of the \\ requirements for the degree of \\ Doctor of Philosophy \\ in \\ Chemistry \\ in the \\ GRADUATE DIVISION \\ of the \\ UNIVERSITY OF CALIFORNIA, BERKELEY \\ Committee in charge: \\ Professor A. Paul Alivisatos, Chair \\ Professor Ignacio Tinoco \\ Professor Lydia Sohn
}

Spring 2005 
The dissertation of Christine Marya Micheel is approved:

\begin{tabular}{ll}
\hline Chair & Date \\
\hline & Date \\
\hline & \\
\hline & Date
\end{tabular}

University of California, Berkeley

Spring 2005 
Biomolecular Assembly of Gold Nanocrystals

Copyright 2005

by

Christine Marya Micheel 


\author{
Abstract \\ Biomolecular Assembly of Gold Nanocrystals \\ by \\ Christine Marya Micheel \\ Doctor of Philosophy in Chemistry \\ University of California, Berkeley \\ Professor A. Paul Alivisatos, Chair
}

Over the past ten years, methods have been developed to construct discrete nanostructures using nanocrystals and biomolecules. While these frequently consist of gold nanocrystals and DNA, semiconductor nanocrystals as well as antibodies and enzymes have also been used. One example of discrete nanostructures is dimers of gold nanocrystals linked together with complementary DNA. This type of nanostructure is also known as a 'nanocrystal molecule.' Discrete nanostructures of this kind have a number of potential applications, from highly parallel self-assembly of electronics components and rapid read-out of DNA computations to biological imaging and a variety of bioassays.

My research focused in three main areas. The first area, the refinement of electrophoresis as a purification and characterization method, included application of agarose gel electrophoresis to the purification of discrete gold nanocrystal/DNA conjugates and nanocrystal molecules, as well as development 
of a more detailed understanding of the hydrodynamic behavior of these materials in gels. The second area, the development of methods for quantitative analysis of transmission electron microscope data, used computer programs written to find pair correlations as well as higher order correlations. With these programs, it is possible to reliably locate and measure nanocrystal molecules in TEM images. The final area of research explored the use of DNA ligase in the formation of nanocrystal molecules. Synthesis of dimers of gold particles linked with a single strand of DNA—possible through the use of DNA ligase-opens the possibility for amplification of nanostructures in a manner similar to polymerase chain reaction.

These three areas are discussed in the context of the work in the Alivisatos group, as well as the field as a whole.

Professor A. Paul Alivisatos

Dissertation Committee Chair 
To my family,

for your constant support 
Only after I had familiarized myself with alchemy did I realize that the unconscious is a process, and that the psyche is transformed or developed by the relationship of the ego to the contents of the unconscious.

—Carl Jung, in Memories, Dreams, Reflections

That is why all the true scientists, the true searchers among the alchemists, say: "I am not looking for the gold of the ordinary people, I am not looking for vulgar gold. I am seeking a higher gold, I am seeking something else."

—Marie-Louise von Franz, in Alchemical Active Imagination 


\section{Table of Contents}

List of Figures

Acknowledgements $\mathrm{v}$

$\begin{array}{ll}\text { I. Introduction } & 1\end{array}$

II. Background: Discrete Nanocrystal/Biomolecule Conjugates and 8 Nanostructures

1 Introduction 8

2 Synthesis and Purification of Gold Nanocrystal/DNA Conjugates 8

3 Synthesis and Characterization of Gold Nanocrystal/DNA 16 Nanostructures

4 Synthesis and Use of Additional Inorganic Nanomaterials 30

5 Synthesis and Use of Additional Biomolecules 33

III. Synthesis and Characterization of Gold Nanocrystal/DNA 41 Nanostructures

1 Introduction 41

2 Discrete Gold Nanocrystal/DNA Conjugate Synthesis and 43 Purification

3 Use of Discrete Gold Nanocrystal/DNA Conjugates in Nanostructure $\quad 57$ Formation

4 Conclusions $\quad 69$

IV. Electrophoretic Mobility Studies of Gold Nanocrystal/DNA 70 Conjugates

1 Introduction $\quad 70$

2 Background $\quad 72$

3 Experimental Methods $\quad 76$

4 Results $\quad 77$

5 Discussion $\quad 87$

6 Conclusions 91

V. Correlation Analysis of Data from Transmission Electron 92 Microscopy

1 Introduction $\quad 92$

2 Methods 93

3 Results 97

4 Conclusions 106

VI. Progress Towards Nano-Amplification 109

1 Introduction 109

2 Experimental Methods 113

3 Results 118

4 Conclusion 129 
VII. Conclusions

1 Biomolecular Assembly of Gold Nanocrystals

2 Prospects for Discrete Nanostructures

3 Conclusions

References 


\section{List of Figures}

2.1 Nanocrystal Molecules 19

2.2 AFM of Au/DNA conjugates $\quad 22$

2.3 Molecular beacons 27

$2.4 \quad \mathrm{Au} / \mathrm{DNA}$ conjugates as remote antennae for inductive heating 29

2.5 Biotemplating of gold particles using proteins 36

2.6 Scattering Experiment 40

3.1 Bis(p-sulfonatophenyl)phenylphosphine, dihydrate, dipotassium 47 salt

3.2 Electrophoresis of discrete Au/DNA conjugates 49

3.3 Non-thiolated DNA does not bind to gold nanocrystals 51

3.4 Controls demonstrating presence of discrete Au/DNA 53 conjugates

3.5 Minimum DNA size is needed in order to effect good 55 electrophoretic separation

3.6 Electrophoretic purification of Au/DNA conjugates and dimers 63

3.7 Electrophoresis of homodimers and heterodimers 64

3.8 TEM images of homodimers and heterodimers 65

3.9 Electrophoresis and TEM of heterotrimers 67

4.1 Characteristics of and electrophoretic forces on gold 73 nanocrystals and DNA

4.2 Electrophoresis of discrete Au/DNA conjugates 82

$\begin{array}{lll}4.3 & \text { Electrophoretic mobility of DNA } & 78\end{array}$

4.4 Electrophoretic mobility of gold nanocrystals $\quad 80$

4.5 Ferguson plot of gold nanoparticles, DNA and Au/DNA 82 conjugates

4.6 Ferguson plot of gold nanocrystals $\quad 84$

4.7 Ferguson plot of DNA oligonucleotides $\quad 85$

4.8 Ferguson plot of various Au/DNA conjugates 86

$\begin{array}{lll}4.9 & \text { Trapping electrophoresis } & 90\end{array}$

5.1 Pair correlations for homodimers 99

5.2 Pair correlations for heterodimers 100

$\begin{array}{ll}5.3 \text { Analysis of Trimers } & 102\end{array}$

5.4 Analysis of Trimers made using branched DNA 104

5.5 Analysis of semiconductor/gold nanocrystal nanostructures 105

5.6 Monte Carlo simulation of pair correlation for homodimers 107

6.1 Schematic of nano-amplification 110

6.2 Tetramer for nano-amplification 111

$\begin{array}{lll}6.3 & \text { Chemical ligation } & 123\end{array}$

6.4 Electrophoresis of 1.4-nm-gold/DNA conjugates 125

6.5 Electrophoresis of PEG-coated Au/DNA conjugates 128

6.6 Electrophoresis and TEM of dimers formed by site-specific 130 aggregation

$\begin{array}{lll}7.1 & \text { DNA nano-barcodes } & 140\end{array}$ 


\section{Acknowledgements}

I am incredibly grateful to the hosts of people who have supported me throughout graduate school.

I would like to thank my family, including my parents Ray and Debbie, who have been supportive of all my decisions, but have always put in their two cents. My brother Bill always seems to thrive wherever he goes, and I wish he would come live near me again. My aunts and grandmother rallied around my mom when she was living through cancer; their love supported me when I was far from home.

I would like to thank my teachers and advisors who inspired me even before college: Beverly Iverson, Ross Iverson, Gerry Huss, Mark Engle, Scott Chumbley, and Rod Western. My undergraduate advisor Bill Buhro has been a continuing and encouraging mentor for many years. I would also like to thank my friends Rachel Popelka, Emily Gubler, Erin Guinness, Amanda Jeter, Carolyn Jones Otten and Anja Mueller for all of their support and reminding me of all the quirky happiness that can be found all around us.

My advisor Paul Alivisatos has always offered insightful and hopeful guidance whenever I needed it most. I would like to thank the members of my qualifying exam and dissertation committees: Ignacio Tinoco, Marcin Majda, Rich Mathies, Mike Chamberlin and Lydia Sohn. During graduate school, many professors and 
staff have offered timely advice and/or opportunities for service to the chemistry department: John Arnold, Laura Landweber, and Rita Tidwell are among many others. Helen Cameron was an outstanding advocate and a strong shoulder for me to lean on when I needed it.

Whole hosts of friends have provided me with incredible support, long-lasting friendships, and many diversions from my day-to-day routine. The Science League-Stephen Prilliman, Benjamin Boussert, Delia Milliron, Deborah Aruguete and Steve Robertson—made my education here both excellent in quality and as fun as possible. I would also like to thank all of my excellent coworkers from the Alivisatos group. Crafts Saturday and Crafts Sunday were pleasant ways to relax, and I learned many valuable lessons from Ryoko Kita. I would like to thank all of my salsa dancing amigos, Rich Yee and the folks at the Works, the SalsaMania Dance Company, the folks at the San Francisco Jung Institute, the women at the Women's Cancer Resource Center, lota Sigma Pi, and all of the other folks who helped me keep my life balanced.

Finally, I would like to thank the Howard Hughes Medical Institute for their generous support through a Predoctoral Fellowship. 


\section{Chapter I}

\section{Introduction}

\section{Discrete Nanocrystal/Biomolecule Conjugates and Nanostructures}

Nanocrystal molecules are analogues to atomic molecules in that nanocrystals comprise the nuclei present in a molecule, and DNA or other biomolecules represent the electronic bonds. Nanocrystals are known as artificial atoms due to their atom-like electronic properties, even though they are made of hundreds to thousands of atoms. By using nanocrystals as nodes in hybrid nanostructures, just as atoms are nodes in molecules, the analogy is extended. Using methods developed over the last 10 years, it is possible to attain discrete 'valencies' for these artificial atoms using different numbers of reactive biomolecules, increasing the potential for constructing interesting new nanomaterials.

Because of the specific yet versatile nature of nanostructure synthesis using nanocrystal/biomolecule conjugates, there is great potential for the application of these structures in the realms of biology and materials science. There are two types of nanocrystal/biomolecule conjugates present in the literature. Most prevalent are those consisting of biomolecule-coated nanocrystal conjugates, especially gold nanocrystals coated with DNA. This includes the excellent work of Mirkin and coworkers [1] that has been reviewed elsewhere [2-5]; for a 
comprehensive review, see reference 6 . The other type of nanocrystal/biomolecule conjugates—and the topic of this dissertation—is discrete conjugates comprised of nanocrystals bound to a small, specific number of biomolecules. In particular, this dissertation focuses on the work I conducted involving nanostructures constructed from gold nanocrystals and DNA, either with or without the use of biological enzymes.

Since the use of discrete conjugates enables highly parallel and directed nanostructure synthesis, it should be advantageous to construct functional devices, quantitative biological probes, fluorescent tags for cell labeling, nanocrystal-based cancer assays, and drug delivery agents, as well as to conduct single-structure experiments such as scattering and single-protein activity studies using discrete building blocks.

Only a few of these applications have been realized to date. The reason for this lies in the inherent challenges of working with nanoscale and biological systems. In the next chapter, a complete review of the work that has been accomplished in the field of discrete nanocrystal/biomolecule nanostructures is presented, as well as discussion of the current challenges facing researchers. All published discrete nanocrystal/biomolecule conjugates, nanostructures and applications thereof known to the author are included. A sampling of other work relevant and directly applicable to the field of discrete nanocrystal/biomolecule nanostructures is included in chapter seven. Particular attention is paid to the characterization 
and synthesis of discrete conjugates, since only a few applications have been realized due to the challenge of synthesizing high-quality conjugates.

\section{Research in the Alivisatos Group}

For now it is useful to summarize the work completed in the Alivisatos group, in order to describe the placement of my work within this context. Researchers in the Alivisatos group began the work in the area of discrete nanocrystal/biomolecule nanostructures. Since 1996, when the first paper was published on these structures [7], the area has flourished and has now grown to include many topics, from continuing work in synthesis of gold nanocrystal/DNA nanostructures [8] to use of semiconductor nanocrystals in cancer cell motility assays [9].

Work in the Alivisatos group has centered on two areas: use of gold nanocrystals in constructing discrete nanocrystal/biomolecule structures and use of semiconductor nanocrystals for optical detection of biological molecules and processes. 1.4-nm, 5-nm, and 10-nm gold nanocrystals have been used to construct dimers, trimers, chains, branched trimers, and branched tetramers connected with DNA [7-8, 10-12]. Antibodies have also been used to create dimers of gold nanocrystals [13]. Many properties of gold nanocrystal/DNA conjugates and nanostructures have also been studied: the hydrodynamic properties of these materials in agarose gels, in particular [14-15]. Computer 
programs have been developed to assist in the analysis of large amounts of TEM data, so that accurate interpretations of the effects of DNA length and other properties on the nanostructures. Gold nanocrystal/DNA nanostructures have been useful in optical scattering experiments [16-17] and have been explored for possible use in DNA computing.

Work on semiconductor/biomolecule conjugates began shortly after the initial work with gold and DNA. CdSe/ZnS core-shell nanocrystals were made watersoluble with a coating of silica, were conjugated to biomolecules, and were used to label cells. The nanocrystals were attached to either anionic silane molecules or the protein avidin and were then bound either to the cell nuclei through an electrostatic interaction or to actin filaments prepared with streptavidin through the specific biotin-streptavidin interaction [18]. The method for coating CdSe/ZnS core-shell nanocrystals with silica was perfected and described by Gerion et al., not long before DNA was successfully attached to the nanocrystals [19]. While it has not been possible to purify the conjugates using gel electrophoresis, as is done for the gold nanocrystal/DNA conjugates, Fu et al., have successfully made discrete semiconductor/gold nanocrystal/DNA hybrid nanostructures. Gold nanocrystals conjugated to exactly one DNA strand are hybridized to semiconductor nanocrystals conjugated to a few DNA strands [20]. The resulting structures were purified using gel electrophoresis and confirmed using TEM. Water-soluble semiconductor nanocrystals conjugated to biomolecules have also 
been used in a variety of biological applications, such as sorting and detection of biomolecules, and a cell motility assay [9, 21-23].

\section{My Research in the Alivisatos Group}

The majority of my own work was conducted in the area of nanostructures made from discrete gold nanocrystal/DNA conjugates. My first project, which has not discussed elsewhere in this dissertation, was to determine whether it would be possible to separate gold nanocrystal/DNA conjugates using isoelectric focusing. This method is commonly used to separate proteins on the basis of their isoelectric point. A pH gradient is formed in a gel, using series of ampholytes and an electric field. Protein samples subsequently migrate to their own isoelectric points in the gel, thus effecting the separation. While this might have been a good method to separate the conjugates, it became evident that the surface stabilization of the gold nanocrystals was not sufficient to withstand the high ionic strength caused by the presence of high concentrations of amphoteric molecules. This project was one of many attempts, which have continued ever since, to find more efficient ways to separate discrete gold nanocrystal/DNA conjugates. Also, the issue of the surface stabilization of the gold nanocrystals is ongoing, and will be discussed in some detail in relation to attempts at amplification of nanostructures. 
After the failure of isoelectric focusing as a separation method for gold nanocrystal/DNA conjugates, I turned back to the use of agarose gel electrophoresis as a separation method for the conjugates and, later, dimers and trimers of gold nanocrystals. This work was done in close collaboration with Daniela Zanchet [11-12]. Since our proof that the bands observed in gels corresponded to gold nanocrystals with differing numbers of DNA strands attached was indirect, I then set about to make quantitative measurements. This work involved the construction and interpretation of Ferguson plots, which are frequently used to illuminate the hydrodynamic properties of biomolecules that are analyzed using electrophoresis. The theories of electrophoresis and polymer hydrodynamics are large and complex, and inspire respect in those who attempt a cursory examination. The knowledge I gained from this work was later helpful in discussions of the work eventually published by Parak et al. [15].

In further attempts to make the characterization of gold nanocrystal/DNA conjugates and nanostructures more quantitative and free from investigator bias, I developed a variety of programs to analyze data obtained from transmission electron microscopy. Eventually, programs were developed to analyze homodimers, heterodimers, trimers, tetramers and even semiconductor nanocrystal/gold nanocrystal/DNA hybrid nanostructures $[8,12,20,24]$. This worked also inspired the development of Monte Carlo simulations of gold nanocrystal/DNA dimers. 
For a number of years, I also participated in a collaboration with several researchers at Princeton and Stanford Universities_-primarily Laura Landweber, Lydia Sohn, and Chris Webb—on the topic of molecular computation using automated microfluidic sensors. The use of gold nanocrystals as labels for particular sequences of DNA could be used to substantially increase the speed of the readout portion of a DNA computation. During the course of this collaboration and other research, it was determined that use of enzymes—restriction endonucleases or ligases, in particular—would be useful for construction and manipulation of nanocrystal/DNA nanostructures. This eventually led to my work on the topic of amplification of gold nanocrystal/DNA nanostructures using DNA ligase, which is discussed in Chapter VI. 


\section{Chapter II}

\section{Background}

\section{Introduction}

This chapter begins with a survey of the methods for synthesizing and purifying discrete gold nanocrystal/DNA conjugates. The second section deals with the synthesis and characterization of discrete nanostructures made using nanocrystals and biomolecules. The third section addresses additional nanocrystals and inorganic materials that have been utilized in discrete structures as well as the additional benefits derived from use of these materials. The conjugates include those comprised of CdTe, and CdSe/ZnS core-shell nanocrystals conjugated to DNA or a protein as the biomolecular component. The fourth section deals with additional biomaterials and their function in discrete structures. It should be noted that my own work, as well as other work from the Alivisatos group, is also reviewed in this chapter.

\section{Synthesis and Purification of Gold Nanocrystal/DNA Conjugates}

The first substantial challenge to address in the assembly of discrete nanobiomaterials is the synthesis of pure nanocrystal/biomolecule conjugates in the highest possible yield. For many reasons, the first and most widely used 
system is that of gold nanocrystals and DNA oligonucleotides. This section surveys the primary methods for the synthesis of gold nanocrystal/DNA conjugates as well as the details concerning gold nanocrystal surface functionalization. The character of the gold surface is critical for optimal binding and activity of biological molecules. Electrophoresis as a method to purify conjugates is also discussed.

There are two primary methods used to synthesize discrete nanocrystal/biomolecule conjugates: (1) statistical mixing of nanocrystals with biomolecules and (2) mixing nanocrystals with functionalized biomolecules followed by purification using electrophoresis. Both of these methods have advantages suited for a variety of nanostructure synthetic methods as well as biological and materials applications, which will be discussed in later sections.

\subsection{Gold Nanocrystal Surface Passivation}

The behavior of DNA conjugated nanocrystals is influenced by interactions between the solvent and the molecules used for passivation of the nanocrystal surface. For instance, gold nanocrystals are synthesized such that they are stabilized with a layer of citrate ions. The negative charge of the ions causes the nanocrystals to repel each other electrostatically, thus overcoming the attractive van der Waals forces and preventing aggregation. 
While citrate stabilization is adequate for pure water $(\mathrm{pH}=7)$ solutions, these nanocrystals tend to aggregate in salt and buffer solutions because of the decreased thickness of the double layer. To address this issue, researchers have coated the nanocrystals with a wide array of alternative passivation agents, including phosphines, polymers, and proteins [25-30]. Within these categories, a wide variety of sizes of molecules may be used to passivate the gold surfaces. Intramonolayer hydrogen bonding can play a strong role in protecting the nanocrystal surface: aromatic interactions provide the best protection, while bulky functional groups has been shown to provide the least protection [31].

While thiolation of DNA at the 5' end, or, less frequently, the 3' end, is the most common way to effect attachment to the gold nanocrystal surface, several other methods offer improvements or alternatives. Tri-thiol capped oligonucleotides can be used to make gold nanocrystal/DNA hybrid materials [32]. These tri-thiol capped oligonucleotides form significantly more stable bonds with gold nanocrystals. Gold nanocrystals coated with tri-thiolated DNA have been observed to be stable in solutions of dithiothreitol for several hours, while those coated with mono-thiolated DNA aggregated within a few minutes [32]. Multithiol anchors could improve stability for any application when reducing agents are present in the solvent, including for optimal use of enzymes. DNA itself can make an excellent passivation layer on gold surfaces, as was shown by Kanaras et al. [28]. 
Non-specific binding of DNA on nanocrystal surfaces is a major issue for the formation of nanocrystal/biomolecule conjugates and nanostructures. It has been observed that the rate of non-specific binding of DNA to gold nanocrystals is length dependent [33] as well as sequence dependent [34]. The problem of non-specific binding has also been addressed for the case of gold nanocrystals by Akerman and coworkers [35] with a study including electrophoretic migration and circular dichroism data. They observed that nonspecifically bound DNA/gold nanocrystal structures can be separated from those of covalently bound DNA/gold nanocrystal structures using electrophoresis. This separation is very useful and utilizes the fact that non-specific binding occurs along the length of the DNA, decreasing the hydrodynamic radii of the conjugates relative to the covalently bound conjugates. Non-specifically bound DNA/gold nanocrystal conjugates therefore migrate more rapidly than their covalent counterparts. The authors noted that their observations of non-specific interaction are different than what we reported in our 2001 Nano Letters paper [11]. In fact, there is not a true discrepancy, since non-specific interaction of DNA with the gold nanocrystals is indeed seen in unpurified gold nanocrystal/DNA mixtures if they are left for more than one to two hours. If electrophoretic purification of conjugates is then attempted after this longer incubation, bands are not observed, and structures do not form through hybridization of complementary conjugates.

Nanocrystal charge has been recognized as a significant factor in the synthesis, conjugation, and application of nanomaterial/biomolecule hybrid materials. 
Neutral gold nanocrystals can be of particular advantage, for their minimal interaction with cellular components, as well as with the biomolecules used for conjugation. For example, Foos et al., reported a variety of ethylene oxide coated, charge-neutral gold nanocrystals that can be subsequently modified with thiols, including thiolated DNA [26]. Other work has shown neutral, polyethylene glycol (PEG) coated nanocrystals [36]. Since it is difficult to functionalize PEGcoated nanocrystals with thiols after PEG coating, subsequent work produced nanocrystals synthesized with biotin-functionalized PEG molecules [30]. While these nanocrystals have not been used to make discrete structures, it may be possible to extend this method into the construction of gold nanocrystal/biomolecule conjugates. The neutral charge of PEG molecules would make nanostructure formation via biomolecular interaction simpler, requiring lower ionic strength and possibly raising yields.

A more drastic approach to manipulating the surface characteristics of gold nanocrystals is to coat them with a functionalizable shell of silica, as is commonly done with Group II-VII and III-V semiconductor nanocrystals [29]. The silica shells can be readily decorated with amino and thiol groups, in much the same fashion as silica-coated semiconductor nanocrystals. This produces a tunable surface in terms of functional group type and number, as well as control over the solubility and exclusion of exchange of pendant groups [29]. In addition to the PEG- and silica-coated nanocrystals discussed above, others have used 
dendrons and polymers to stabilize noble metal as well as semiconductor nanocrystals [37].

\subsection{Formation of Gold Nanocrystal/DNA Conjugates via Statistical Mixing}

In nanocrystal/biomolecule conjugate preparation, statistical mixing describes an approach in which biomolecules are mixed with a large excess of nanocrystals, such that some of the resulting nanocrystals are conjugated with one biomolecule, most are free nanocrystals, and only a very few are conjugated to more than one biomolecule. The primary disadvantage of this method is that without purification it is not possible to obtain relatively pure, higher-order conjugates (i.e. those with more than one biomolecule attached). The use of statistical mixing to create nanocrystal/biomolecule nanostructures was introduced in 1996 using 1.4-nm nanocrystals [7]. This method was used again, though for larger gold nanocrystals ( 5 and $10 \mathrm{~nm}$ ), in 1999 for the synthesis of a variety of nanocrystal molecules [10].

Nanogold $^{\mathrm{TM}}$ (Nanoprobes, Yaphank, NY), 1.4-nm gold derivitized with a single maleimide or one of a few other functional groups, was the first material that provided a simple means to forming discrete nanocrystal/biomolecule conjugates [38-39]. While it may not be obvious why these conjugates should be included in the section on conjugates made using statistical mixing, it turns out that these materials are synthesized in just such a manner. The monofunctionalized 1.4-nm 
gold nanocrystals are synthesized using appropriately derivitized phosphines and are purified using ion exchange chromatography [40]. In their initial applications, these nanocrystals were used as immunoprobes for microscopic analysis of biological tissues. The use of colloidal gold in biology was well established long before the beginning of nanoscience; that use is the primary application of these 1.4-nm nanocrystals even today [41]. There are a number of advantages when using small 1.4-nm nanocrystals: they can be run in polyacrylamide gels and in commonly-used agarose gels [38], and silver enhancement has been utilized to aid visualization of the nanocrystals [39]. It is fortunate that these nanocrystals are modified with a variety of reactive groups, including $\mathrm{N}$-propylmaleimide and N-hydroxy-2,5-dioxopyrrolidine-3-sulfonic acid sodium salt (sulfo-NHS), among others; these different functional groups have been used for a variety of nanocrystal/biomolecule conjugates [7, 42].

Larger nanocrystals have been frequently used for gold nanocrystal/DNA conjugates. This was accomplished for the first time by our group in 1999, when 5- and 10-nm nanocrystals were mixed with thiolated DNA [10]. In addition, Foos et al. have developed a method for creating charge-neutral, statistically mixed single DNA/gold nanocrystal conjugates [27]. They pointed out that statistical mixing can be used to create conjugates with DNA shorter than 50 bases, currently the size limitation for electrophoretically purified gold nanocrystal/DNA conjugates. 
Statistical mixing is an easy, effective method for obtaining functionally pure mixtures of monoconjugates for certain applications. Because of these advantages, this method will find numerous future applications.

\subsection{Electrophoretically Purified Conjugates}

Electrophoretically purified conjugates are synthesized by mixing functionalized biomolecules with nanocrystals, incubating them to allow attachment, and finally purifying the conjugates using gel electrophoresis. Because attachment of biomolecules to nanocrystals can yield conjugates with substantially different size and/or charge, it is often possible to resolve nanocrystals bearing different numbers of biomolecules into distinct bands in a gel. The use of electrophoretically purified conjugates enables the use of larger gold nanocrystals for conjugate and nanostructure synthesis; these larger gold nanocrystals, typically 5 and $10 \mathrm{~nm}$ in diameter, have advantages over smaller nanocrystals for the formation of gold nanocrystal/DNA conjugates and nanostructures, including ease of handling, visualization, surface modification, and conjugation to biomolecules. Even so, gold nanocrystals larger than $1.4 \mathrm{~nm}$ were not used to build discrete structures until 1999 [10-11]. The use of highly colored 5- and 10-nm gold colloids enabled more straightforward electrophoretic separations, since conjugates and nanostructures could be visualized without further staining. 
Higher-order discrete conjugates bearing two or more biomolecules are useful building blocks for the synthesis of more complex nanostructures. While several approaches have been developed for the isolation of gold nanocrystal/biomolecule monoconjgates, gel electrophoresis currently provides the best route to discrete higher-order gold nanocrystal/biomolecule conjugates. Using this method, it has been possible to isolate gold nanocrystals with two, three, or more DNA strands attached (see Figure 3.2) [11]. In this method, the biomolecule (DNA from 50 to 100 bases in length in most cases) is mixed with nanocrystals (phosphine-coated 5- or 10-nm gold nanocrystals in most cases) and incubated for one to three hours. Subsequently, the conjugate mixture is run on an agarose gel, wherein the conjugates are separated by size. While electrophoretic separation is labor-intensive and sometimes low-yielding, it allows for the construction of materials more complex than dimers of nanocrystals. Alternatively, conjugates can be separated from the gel using a crush-and-soak method that allows conjugates to diffuse into a buffer solution.

\section{Synthesis and Characterization of Gold Nanocrystal/DNA Nanostructures}

The challenge involved in creating nanostructures from discrete gold nanocrystal/DNA conjugates is similar to that involved in creating the conjugates themselves. Scientists are faced with similar challenges of non-specific absorption of DNA on gold surfaces, low yields, and difficult-to-characterize products. This section surveys the structures that have been synthesized as well 
as characterization methods that have successfully helped to illuminate the properties of the new nanostructures. Applications of these structures are also discussed.

\subsection{Synthesis}

The nanostructures made using discrete nanocrystal/biomolecule conjugates have been increasing in complexity and applicability over the past 10 years. The first discrete nanocrystal/biomolecule nanostructures were made using Nanogold ${ }^{\mathrm{TM}} / \mathrm{DNA}$ conjugates. The first was a paper that presented the original discrete gold nanocrystal/DNA nanostructures. Head-to-head dimers, head-totail dimers, and parallel trimers were demonstrated by Alivisatos, Schultz et al., in 1996 [7]. Since that time, more nanostructures have been synthesized using these convenient monofunctionalized nanocrystals. In one example, the attachment of 1.4-nm gold nanocrystals to a tiled DNA lattice was demonstrated [43]. The spacings between the gold nanocrystals are 2 and $64 \mathrm{~nm}$ and are visualized using atomic force microscopy (AFM) and scanning TEM. The gold nanocrystals are incorporated prior to lattice formation by covalent attachment to one of the DNA strands. Reif and coworkers made a quadruple crossover DNA array, and then attached either streptavidin or streptavidin-functionalized Nanogold ${ }^{\mathrm{TM}}$ along one or both sides [44]. Hairpin loops on the lattices incorporated biotin functional groups for the streptavidin binding. 
Using statistical mixing to produce unpurified gold nanocrystal/DNA conjugates, it is possible to construct discrete nanostructures (Figure 2.1) [10]. While the gold nanocrystal/DNA conjugate starting materials were not purified, the resulting structures were purified using gel electrophoresis. Many different structures were constructed, including template-directed heterodimers and homotrimers, conjugate-templated homotrimers and heterotrimers, and interior-thiol heterotrimers. The interior-thiol heterotrimer structures are unique in that trimers are made, yet no multiple DNA conjugates are required: one gold nanocrystal is conjugated to a DNA strand with the thiol in the middle and the other two gold nanocrystal/DNA conjugates hybridize to the DNA on either side of the thiol.

There is one example of nanostructure synthesis using gold nanocrystals with approximately two DNA strands per nanocrystal. Nanocrystal rings were formed using two complementary sets of DNA-modified gold nanocrystals [45]. These rings are formed by similar mechanisms as those formed by solvent evaporation alone; however, unlike rings formed by solvent evaporation alone, DNA hybridization greatly expands the range of diameters of the rings, as well as creates rings with single-particle thickness. This method could give researchers the ability to better control the nanocrystal alignment in the absence of controlled attachment angles of biomolecules to nanocrystals and the effects of flexible linker components. 


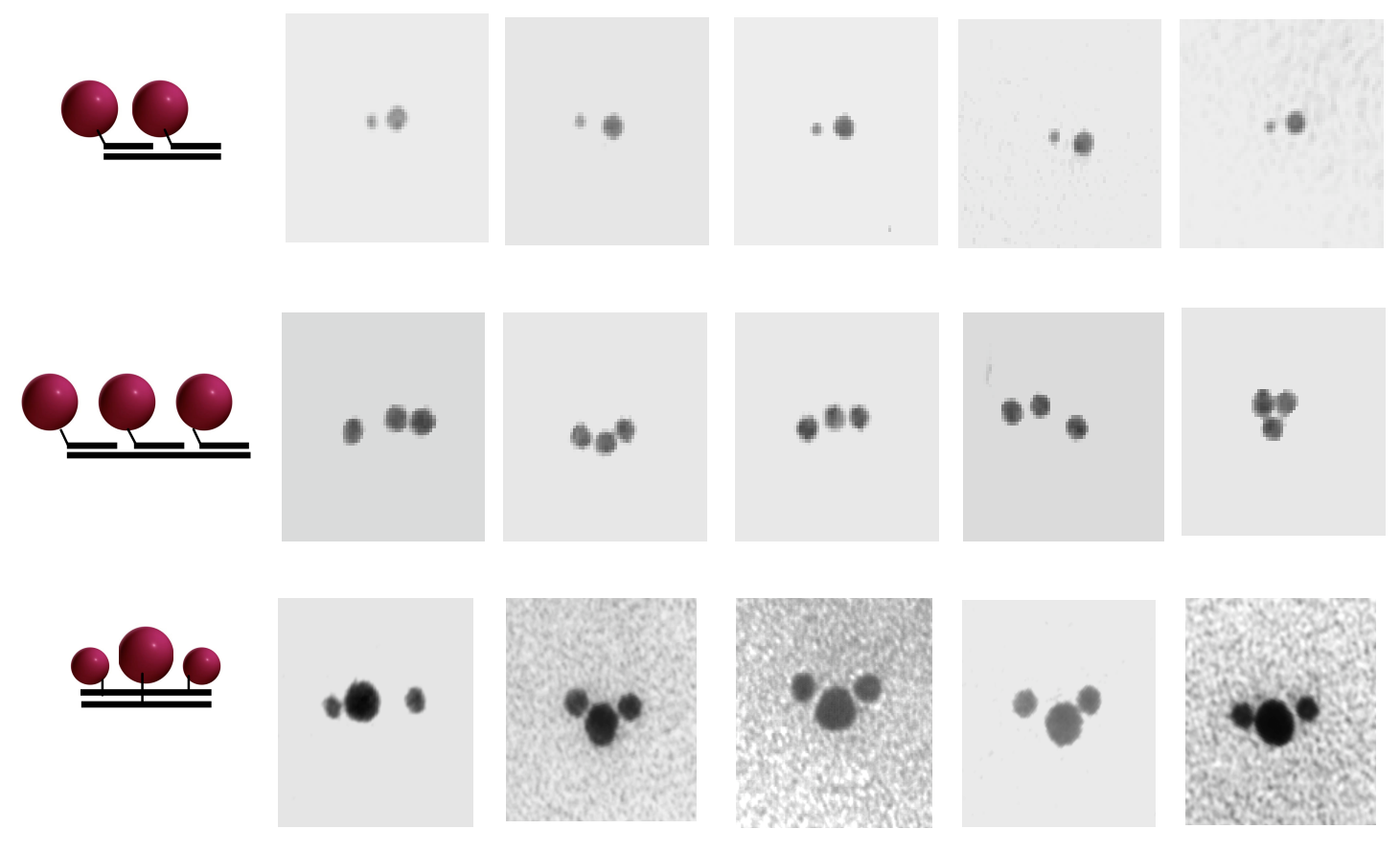

Figure 2.1 Nanocrystal Molecules. Transmission electron microscope images of gold nanocrystal/DNA conjugates annealed to a DNA template strand (top two series) or to another conjugate (bottom series). Reprinted with permission from Loweth, C.J., Caldwell, W.B., Peng, X.G., Alivisatos, A.P., and Schultz, P.G. Angew. Chem. Int. Ed. 18, 1808-1812 (1999). 
There are two instances in the literature that use electrophoretically purified gold nanocrystal/DNA conjugates to synthesize nanostructures; in one, synthesis of dimers and trimers of nanocrystals made from discrete gold nanocrystal/DNA conjugates was shown [12]. These form in much higher yield than structures synthesized from mixtures of conjugates (see Figure 3.9). Homodimers, heterodimers, homotrimers and heterotrimers were presented. After the conjugates are purified, complementary conjugates are mixed together with appropriate stoichiometry, allowed to incubate overnight, and then purified using agarose gel electrophoresis. Very long DNA has also been attached to larger 13-nm nanocrystals and purified electrophoretically to yield gold nanocrystals attached to one or two DNA strands [46].

\subsection{Characterization}

Because of the challenges that have arisen in synthesizing more complex nanocrystal/biomolecule hybrid materials, much work has been done to understand the properties of nanocrystal/biomolecule conjugates-gold nanocrystal/DNA conjugates and nanostructures in particular. Characterization techniques include imaging and analysis of conjugates and nanostructures, studying hydrodynamic properties of conjugates and nanostructures, and exploring nanocrystal and biomolecule stability and binding mechanisms. 


\subsubsection{Imaging}

Direct visualization of the components making up a nanocrystal/biomolecule hybrid material is challenging. Numerous methods are used for observing one or both of the components, including electron microscopy, scanning force microscopy (SFM), and near-field scanning optical microscopy (NSOM). A few researchers have specifically addressed visualization of nanocrystal/biomolecule hybrid structures [47]. The groups of Niemeyer, Seeman, and Reif are particularly skilled at the use of AFM to observe DNA nanostructures [43, 48-49]. Several groups have also made observations of gold nanocrystal/DNA nanostructures using AFM (Figure 2.2) [46, 50].

TEM has been the central imaging technique used to visualize nanostructures in the Alivisatos group and in others. Imaging and analysis methods have been steadily improving since the first papers on gold nanocrystal/DNA nanostructures, starting with simple images of structures, and building to the development of a number of analytical methods for analyzing this data [12]. This is important for discrete nanostructures, since the yields can be low, and the eye can be easily biased. Two main types of TEM analysis programs have been developed. The first program analyzes distance correlations between nanocrystals and can be used to analyze homodimers as well as heterodimers by either comparing a particular size of nanocrystal with itself, or two sizes of nanocrystals to each other (see Figure 5.1). The second type of program looks 


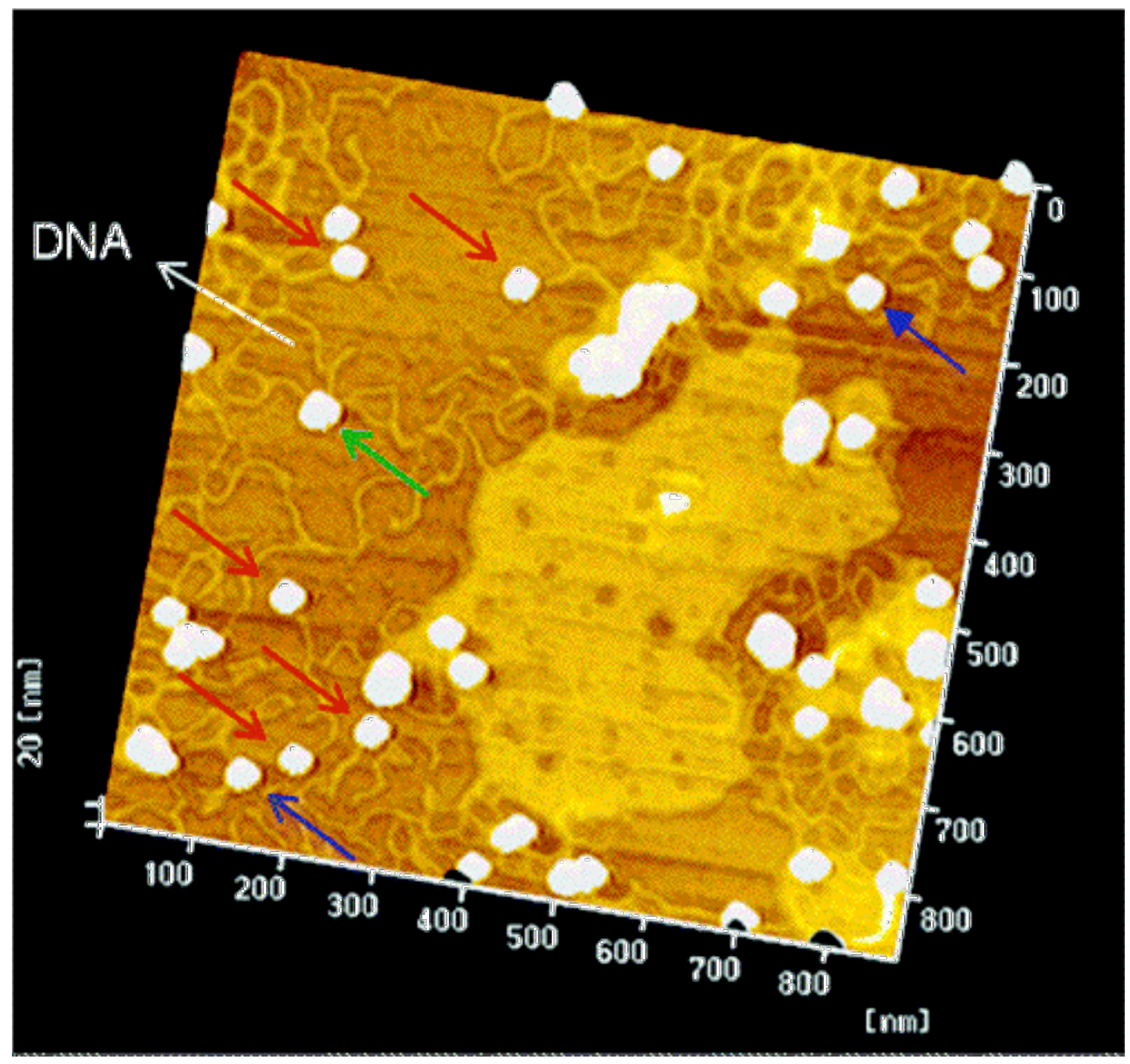

Figure 2.2 AFM image of gold nanocrystal/DNA conjugates. The arrows indicate examples of gold nanocrystal/DNA conjugates (red: gold + 1 DNA, blue: gold + 2 DNA, green: gold + 3 DNA). The average diameter of the gold nanocrystal is $37.3 \pm 2.8 \mathrm{~nm}$ and the DNA length is $1.7 \mathrm{~kb}$. Reprinted with permission from Tsai, C.-Y., Shiau, A.-L., Cheng, P.-C., Shieh, D.-B., Chen, D.-H., Chou, C.-H., Yeh, C.-S., and Wu, C.-L. Nano Letters 4, 1209-1212 (2004). Copyright 2004 American Chemical Society. 
for correlations of specific numbers and sizes of nanocrystals. This type of program is critical for the analysis of structures bearing more than two nanocrystals, since pair distributions only show correlations between pairs of nanocrystals. This program has been used to analyze trimers, tetramers, and pentamers of nanocrystals, and it can also be used to compare structures made from multiple sizes of nanocrystals $[8,20,24]$. Others have made use of interparticle correlations, though these measurements have not been automated and so have many fewer nanocrystals represented [51].

\subsubsection{Hydrodynamic Properties}

In the first electrophoretic studies of gold nanocrystal/DNA conjugates, which explore some of the hydrodynamic properties of these complex hybrid materials, the gel percentage, the gold size, and the DNA length were varied to look for trends in the electrophoretic mobilities of the conjugates, as well as to explore the hydrodynamic behavior of these unusual polymers [12]. As determined by Ferguson plot analysis, gold nanocrystal/DNA conjugate behavior closely matches that of streptavidin/DNA conjugates, which are used in a method called trapping electrophoresis [52-56]. The significance of work on trapping electrophoresis-which was initially explored as a possible way to separate very large DNA strands, a role now assumed by pulsed field gel electrophoresis-is that it illuminates the mechanism of electrophoresis, giving clues as to the 
behavior of other nanocrystal/biomolecule hybrids, as well as the behavior of hybrids bearing different relative charges.

Additional electrophoretic studies of gold nanocrystal/DNA conjugates explored the hydrodynamic properties of gold coated with varying numbers and lengths of DNA [15]. The hydrodynamic radius of a gold nanocrystal/DNA conjugate was observed to increase linearly with the length of DNA for nanocrystals entirely coated with DNA, up to a length of 30 bases. Above 30 bases, the DNA segments are very flexible in solution and contribute significantly less to the overall hydrodynamic radius. As observed in gold nanocrystals coated with only a few DNA strands, electrophoretic mobility is more rapid than expected due to the fact that the DNA wraps around the nanocrystal surface. Even sequence dependent mobility was shown, with conjugates rich in T and A migrating more rapidly than conjugates low in $\mathrm{T}$ and $\mathrm{A}$.

\subsubsection{Other methods}

A variety of other methods have been used to characterize gold nanocrystal/DNA conjugates and nanostructures, including dynamic light scattering (DLS), static light scattering (SLS), small angle X-ray scattering (SAXS), and measurements using a quartz crystal microbalance. 
Dynamic light scattering (DLS) is a method used by a number of researchers to examine the hydrodynamic properties of nanocrystal/biomolecule hybrid materials. This method has the advantage that it can probe the hydrodynamic properties of these materials in the absence of a gel and electric field, both of which perturb the properties of the nanostructures. In one example, the authors developed the use of biotin-covered gold nanocrystals and free streptavidin/DNA conjugates to make gold nanocrystal/DNA conjugates [57]. Complementary gold nanocrystal/streptavidin/DNA particles are mixed together, and the familiar threedimensional networks result. Using DLS, they measured the hydrodynamic radii of particles in various stages of biomolecule conjugation with biomolecules and nano-assembly.

Other methods that give information about nanocrystal/biomolecule conjugates and nanostructures include small-angle X-ray scattering (SAXS), static light scattering (SLS), and UV/Vis spectroscopy. These methods, along with transmission electron microscopy (TEM), DLS, and NMR (for quantifying ligand binding to nanocrystals) are compared in a paper by Fitzmaurice and coworkers [58]. In particle sizing, SAXS and SLS are limited by difference in electron density between material and solvent as well as sample polydispersity. DLS is limited by correlation time and wavelength of light used. TEM is limited by sample preparation and instrument resolution. NMR is used to characterize modified gold nanocrystals; molecules bound to gold surfaces are not detected, hence it is a negative characterization method. UV/Vis is good at determining 
aggregation, and some of the sizing methods are also useful for determining aggregate size. In addition, UV/Vis spectroscopy has shown that optical spectra of discrete nanostructures are not seen to shift in solution, as they are in the aggregate structures [10].

\subsection{Applications}

Many current applications of gold nanocrystal/DNA conjugates and nanostructures make use of the unique optical and electronic properties of gold nanocrystals [3, 59-63]. For example, several of the biological applications of discrete gold nanocrystal/DNA conjugates use the greater efficiency of gold nanocrystals relative to conventional organic dye molecules as quenchers of molecular fluorescence. For example, DNA with self-complementary extremities, a molecular beacon, is attached to a 1.4-nm nanocrystal on one end and a fluorophore on the other end; it is sensitive to conditions in solution as well as presence of complementary sequences (Figure 2.3) [64]. In this experiment, researchers added the strand complementary to the middle section of the molecular beacon, and fluorescence was restored to the solution. As mentioned, gold nanocrystals quench fluorescence better than organic quenchers, giving onoff intensity ratios of several thousand. Fluorophores with an affinity for gold nanocrystal surfaces have also been used [65]. In the absence of a complementary strand, fluorescence was quenched, and in the presence of a complementary strand, the solution fluoresced. 
A

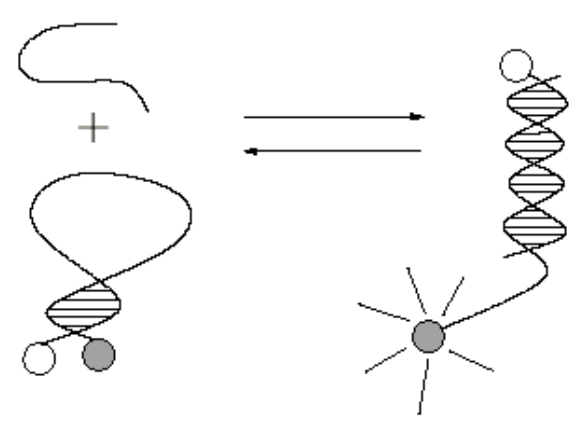

B

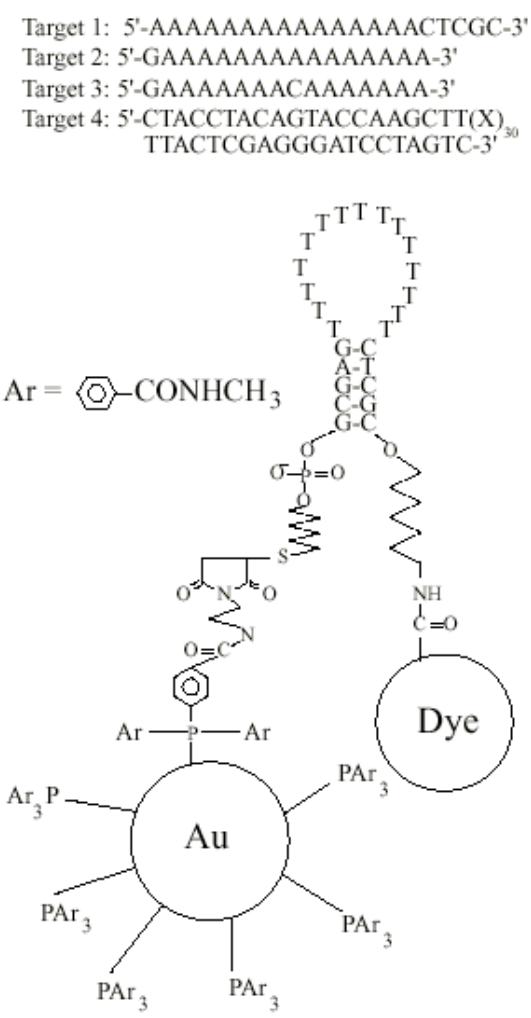

Figure 2.3 Schematic drawings of the two conformations of the dye-oligonucleotide-gold conjugate and of the gold-quenched molecular beacon. (A) The formation of the hairpin (left) results in quenching of the dye. Hybridization to another DNA strand removes the hairpin, and the dye fluoresces (right). (B) DNA sequence, attachment and gold surface chemistry. Reprinted with permission from Dubertret, B., Calame, M., and Libchaber, A.J. Nature Biotech. 19, 365-370 (2001). 
Some controversial work suggests that nanocrystals may also be used as remote antennae for the manipulation of conditions near tagged biomolecules. To demonstrate this capability, DNA oligonucleotides were attached to 1.4-nm gold nanocrystals [66]. Using a rapidly switching magnetic field, the nanocrystals are heated inductively, causing the DNA to dehybridize. Multiple cycles of dehybridization and rehybridization can be realized in the space of minutes, and heating is constrained to the vicinity of the nanocrystal, so nearby molecules are relatively unaffected (Figure 2.4). Large magnetite particles are injected in to tumors and heated to help treat cancers. If verified, this smaller version could be used for studying enzyme activity, conducting DNA computations, and targeted gene therapy.

Gold nanocrystal/DNA conjugates are useful for in situ experiments, such as in situ hybridization (ISH) [67]. ISH is used to detect locations of specific DNA or RNA sequences in cells. Unlike other methods for ISH, this method, when combined with one of several amplification techniques, such as silver enhancement of 1.4-nm gold nanocrystals, can achieve single-copy detection with a 10 - to 50 -fold improvement in sensitivity.

In addition, 1.4-nm gold nanocrystals have been used as a contrast agent for biological electron microscopy for many years, generally through conjugation of appropriate antibodies or protein fragments [68]. Gold nanocrystals are also an effective tag for detection of biomolecules and/or binding events in solution. It is 
a
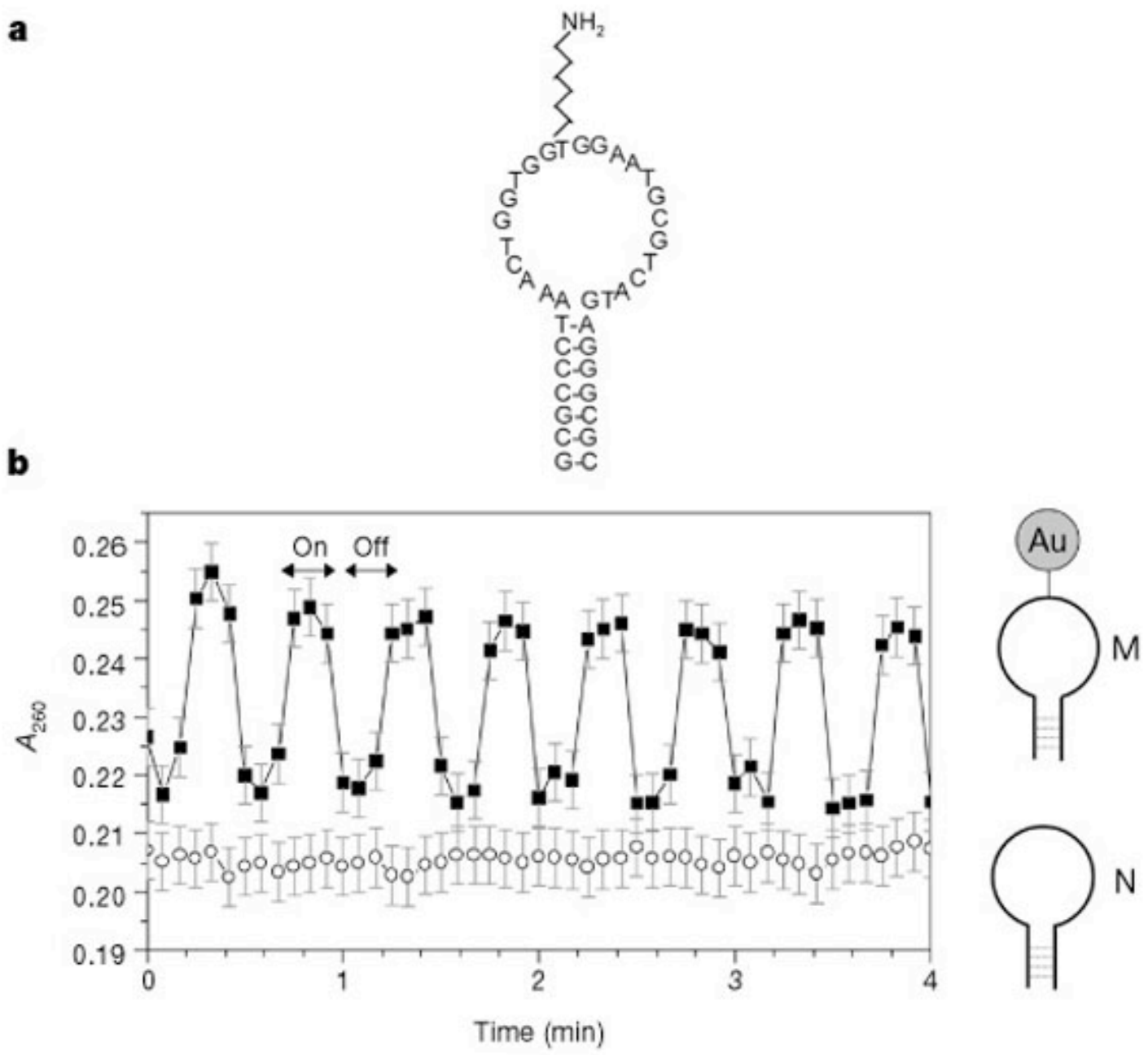

Figure 2.4 Inductive coupling to nanocrystals linked to DNA and evidence of dehybridization. (A) Sequence of the hairpin molecule, M. (B) Absorbance at 260 $\mathrm{nm}$ of a solution of M in RFMF (squares). Arrows indicate when the RFMF is on/off. Circles, response of N (no nanocrystals) in RFMF. Reprinted with permission from Hamad-Schifferli, K., Schwartz, J.J., Santos, A.T., Zhang, S.G., and Jacobson, J.M. Nature 415, 152-155 (2002). 
also possible to determine whether the nanocrystals are attached to a biomolecule, and, in many cases, the orientation of the biomolecule [69].

\section{Synthesis and Use of Additional Inorganic Nanomaterials}

\subsection{Nanocrystal Synthesis}

To realize the full breadth of possible biological and materials applications of discrete nanocrystal/biomolecule conjugates, it is important to expand the realm of discrete nanocrystal/biomolecule structures to include a wide range of nanocrystal materials and surface chemistries. An important aspect of this is the generation of new water-soluble nanocrystals. While the synthesis of the simplest water-soluble citrate-stabilized gold nanocrystals is well understood [41], extending the scope of this system to create nanocrystals with a variety of charges and materials has resulted in a great deal of new science. As a result, there have been a variety of new synthetic routes for nanocrystals presented in the literature.

There have been several examples of water-soluble CdSe/ZnS core/shell nanocrystals used for nanocrystal/biomolecule hybrid structures. There are a number of different procedures for synthesis of water-soluble semiconductor nanocrystals, including silica coating as well as coating with polymers [18-19, 37]. In addition, a variety of work involving CdTe nanocrystals has been 
published. Kotov and coworkers developed a method for synthesizing watersoluble CdTe nanocrystals, which used L-cysteine as a surface-passivating layer [70]. In a recent paper, Weller and coworkers show the synthesis of watersoluble, silica-coated CdTe nanocrystals [71]. These nanocrystals have been successfully conjugated to both biotin and DNA. Gel electrophoresis of CdTe/DNA conjugates show promise for the purification of discrete conjugate structures; gels showed remarkably well-defined nanocrystal bands.

The extension of these methods to new types of nanocrystals-magnetic nanocrystals, for example-and nanocrystal surface chemistries will greatly improve the prospects for future applications of discrete nanocrystal/biomolecule structures.

\subsection{Applications}

New nanocrystals open up new possibilities for biological and materials applications of discrete nanocrystal/biomolecule nanostructures. These include fluorescence labeling for imaging in situ, in arrays for a variety of bio-assays, and for construction of semiconductor/gold nanostructures for optical studies.

Fu et al. utilize gold nanocrystals as satellites to semiconductor nanocrystals [20]. Gold has well-known electronic properties that could lead to quenching or enhancement of the semiconductor nanocrystal fluorescence. It will be 
interesting to see how this ongoing work will compare with that conducted with semiconductor nanocrystals at gold surfaces [72-73]. The effect of distance on quenching of nanocrystal fluorescence has been tested using different lengths of DNA linking 1.4-nm gold nanocrystals to CdSe/ZnS core-shell nanocrystals [74]. It was shown that increasing the DNA chain length caused increased quenching.

Semiconductor nanocrystals with multiple DNA strands attached have been used for several applications. Discrete semiconductor nanocrystal/DNA conjugates have not been synthesized; nonetheless, when a method is developed, use of discrete conjugates would allow quantification of bound biomolecules in solution and thus not be restricted to surfaces. In one of these papers, semiconductor nanocrystal/DNA conjugates have been used to detect single-nucleotide polymorphisms (SNPs) on the p53 gene, which is associated with many types of cancer [23]. Semiconductor nanocrystals have numerous advantages over conventional organic dyes, including broad absorption and high resistance to photobleaching. While these conjugates are not discrete, under optimal conditions the use of discrete conjugates could allow for the quantification of labeled molecules in a cell or solution, a fluorescent version of ISH currently accomplished using gold nanocrystals. Semiconductor nanocrystals conjugated to DNA are also excellent labels for DNA microarrays and gene mapping because of their broad excitation spectrum and their long photostability. 


\section{Synthesis and Use of Additional Biomolecules}

Nanostructures incorporating biological and inorganic materials have expanded to include many types of biomolecules including branched DNA and a wide variety of proteins. In addition to many biological applications, the precise and tunable properties of biological molecules make them ideal for materials applications, acting as information carriers, templates for nanocrystal formation,

and rigid or dynamic structural components. This section discusses the types of biomolecules used and the applications realized to date.

\subsection{Synthesis}

Many of the new biomolecules have been incorporated into nanostructures through attachment to gold nanocrystals, using either a thiol or a maleimide linkage. Even so, some of the new structures use semiconductor nanocrystals such as CdTe.

Branched DNA has been used to construct trimers and tetramers of gold nanocrystals $[8,75]$. Electrophoretic purification of these structures is arduous and highlights the synthetic challenges of complex hybrid structures [8]. Nonetheless, using branched DNA could be an excellent approach to the synthesis of more complex gold nanocrystal/DNA nanostructures, and builds on work of Southern and others who synthesized dendrimeric DNA structures [76]. 
Restriction enzymes have been used to manipulate Nanogold ${ }^{\mathrm{TM}} / \mathrm{DNA}$ dimers [51]. Also, the authors presented the use of a methylating enzyme to manipulate the DNA conformation between the two gold nanocrystals. Conjugates of long DNA attached to 13-nm gold nanocrystals have been electrophoretically purified. The DNA in these conjugates remains active to enzymes such as restriction enzymes [46].

Many small proteins have been used to modify nanocrystals, especially biotin. For example, gold nanocrystals functionalized with just a few biotins or just a few glutathione molecules have been synthesized [77]. In addition, N. Kotov and coworkers demonstrated production and electrophoretic distinction of discrete bovine serum albumin (BSA)/CdTe conjugates [70]. BSA has been extensively studied, since as a plasma protein it can be used to study blood and immune responses of organisms. Also, it was shown that proteins can retain much of their structure and function after conjugation to nanocrystals.

Recently, the electrophoretic purification of discrete nanocrystal/biomolecule conjugates has been extended to gold nanocrystal/antibody conjugates. In recent work, J. Cha synthesized discrete antibody/gold nanocrystal conjugates, which were used to create discrete nanostructures, including heterodimers and heterotrimers of $10-$ and $25-\mathrm{nm}$ gold nanocrystals [13]. It is not well understood how the proteins attach to the nanocrystals, but the bond does not appear to be a labile one. In addition, since there are two active sites per antibody, mixtures of 
discrete conjugates intended to form discrete nanostructures result in formation of the discrete nanostructures as well as polymeric materials where the two active sites link together two gold nanocrystals rather than one. This polymerization limits the applicability of these gold nanocrystal/antibody conjugates, unless a method can be developed for attachment of just one active group or for deactivation of one of the functional groups.

\subsubsection{Biotemplating of Nanocrystals}

Whitesides and coworkers produced a fascinating example of biomolecular templating of nanocrystals [78]. Discrete structures of anti-FITC IgG proteins were made by assembly with bifunctional FITC molecules, and then gold nanocrystals were synthesized at the carbohydrate region of the $\lg G$ proteins (Figure 2.5). This is a novel approach to creating nanocrystal/biomolecule hybrid materials and effectively results in discrete nanostructures. Although the nature of the IgG/FITC interaction may limit the length scale of structures formed purely by this method, more long-range ordering may be achieved by combining this technique with others, such as use of DNA-binding proteins.

Biotemplating has also been used to synthesize magnetite nanocrystals [79] and platinum nanocrystals [80] along the length of DNA. Nickel oxide nanocrystals have been synthesized with the assistance of bovine serum albumin [81], and tellurium wires have been synthesized with the assistance of alginic acid. Much 

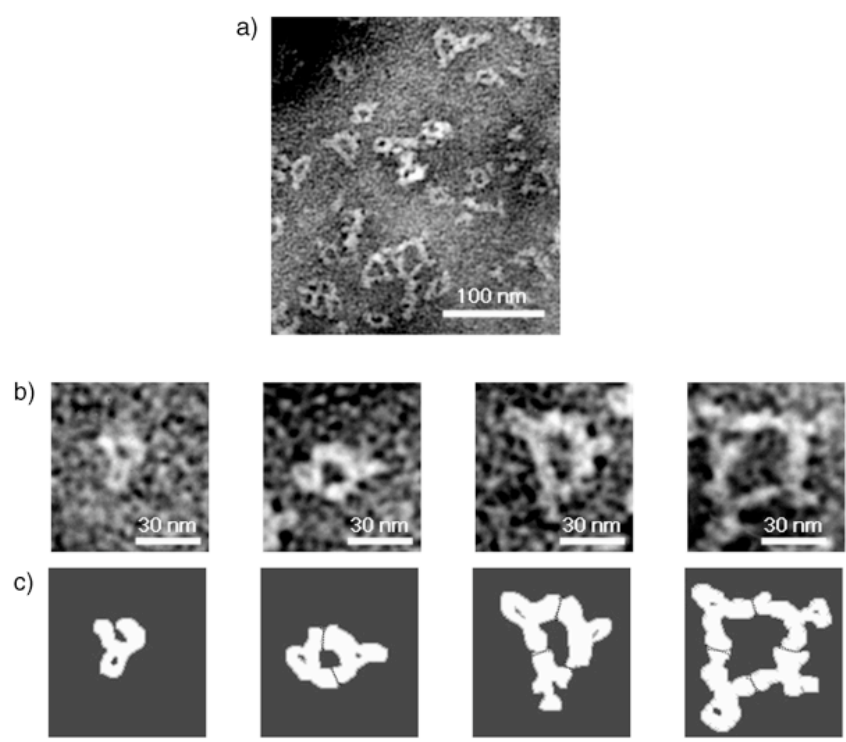

d)
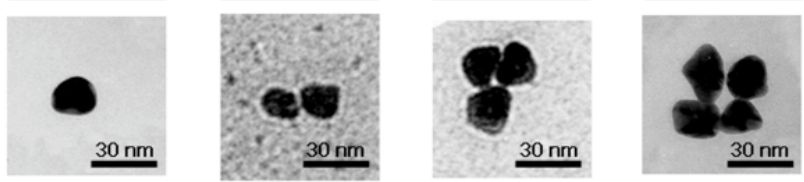

e)

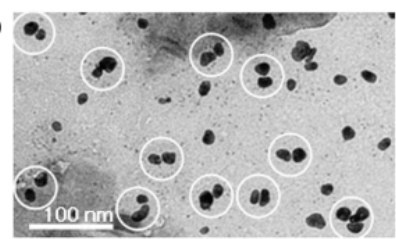

Figure 2.5 TEM images of aggregated antibodies. a) Image of IgG antibodies after incubation with antigen. b) Magnified images of (from left to right) an IgG molecule, cyclic dimer, cyclic trimer, and cyclic tetramer. c) Schematic representation of the corresponding IgG monomer, dimer, trimer, and tetramer in row (b). d) Magnified images of (from left to right) gold nanoparticles presumably grown from a single IgG molecule, a cyclic dimer, a cyclic trimer, and a cyclic tetramer. e) Larger area of a sample of IgG deposited in the presence of antigen after they had been subjected to the metal-growth conditions fragments. Reprinted with permission from Yang, J., Mayer, M., Kreibel, J.K., Garstecki, P., and Whitesides, G.M. Angew. Chem. Int. Ed. 43, 1555-1558 (2004). 
of this work has been inspired in part by research occurring in the area of biomineralization. Biotemplating appears to be an extremely versatile method for the expansion of the synthesis of nanocrystal/biomolecule hybrid materials.

\subsection{Applications}

Gold nanocrystal/biomolecule conjugates have been used for many years in electron microscopy, but in recent years, discrete nanocrystal/biomolecule hybrid materials have found use in other areas of biology as well as materials science.

Recently, monofunctionalized 1.4-nm gold nanocrystals have been used as a tag for electron microscopy studies of RNA molecules in cells [82]. Because the gold nanocrystals allow for attachment of a single functional group, it is possible to track the RNA in a cell. In this study, the clusters could be attached to thiolated uridines in the RNA. This allowed for the attachment of up to five clusters per RNA. TEM analysis showed RNA molecules decorated with gold clusters, evenly spaced along the length of the RNA.

Nanocrystal/protein conjugates can also play an important role in biological applications beyond those involving microscopy. One paper exemplifying this potential describes methods for exploring the bioactivity of proteins attached to gold nanocrystals [83]. There is great potential for studying enzyme and other protein activity in vivo using nanocrystal labels, work that would also help 
determine whether the presence of nanocrystals will adversely distort the function of the proteins. The perturbations brought about by nanocrystals could be harnessed for medical therapies.

Discrete nanocrystal/biomolecule conjugates can also play an important role in tracking of cellular components during in situ experiments. Gold-labeled DNAPNA hybrids have been delivered into the mitochondrial compartments found in cells [84]. This work paves the way for more versatile cell labeling experiments by allowing labeled nanocrystals access to areas of cells that are not accessible by typical cell entry mechanisms such as phagocytosis.

\subsubsection{Scattering and Optical Experiments}

Colloidal nanocrystals as well as many biomolecules have interesting optical properties, ranging from plasmons and fluorescence to complex interparticle interactions. Study of nanocrystal/biomolecule conjugates and nanostructures will yield information about the interactions of these materials, as well as information for the application of these materials for biological and materials sciences.

The fluorescence of a material is strongly affected by its surroundings. Effects of surface chemistry on fluorescence are well understood, and nanocrystal fluorescence has been well studied for several decades. Recently, scientists 
have begun studying the effect of bound biomolecules on nanocrystal fluorescence. BSA has been found to enhance the luminescence of CdTe nanocrystals by resonance energy transfer to the nanocrystals [70], as confirmed by absorbance and emission spectra. This results in a two to three-fold increase in nanocrystal luminescence. The authors assert that this is essentially a communication link between biological and inorganic substances, and can potentially be used for electronics applications.

Single molecule scattering experiments allow observation of nanostructure formation in solution, as well as characterization of nanostructures whose optical properties are difficult to probe in solution due to low concentration and/or significant impurities [85]. This is especially useful for structures made from highly scattering materials, such as gold and silver. Indeed, it is possible to observe formation of gold/silver dimers on surfaces using this technique. The spectra give additional information about the distance between the gold and silver nanocrystals, and this is called a "plasmon ruler" [16]. In other work, researchers have followed binding of streptavidin to biotin-conjugated gold nanocrystals by watching the spectra of the nanocrystals shift [17]. This is extendible to other discrete nanocrystal/biomolecule nanostructures (Figure 2.6). 


\section{(a) Biotin S2 Streptavidin (acceptor) 05 (analyte) \\ Biotinylated BSA}
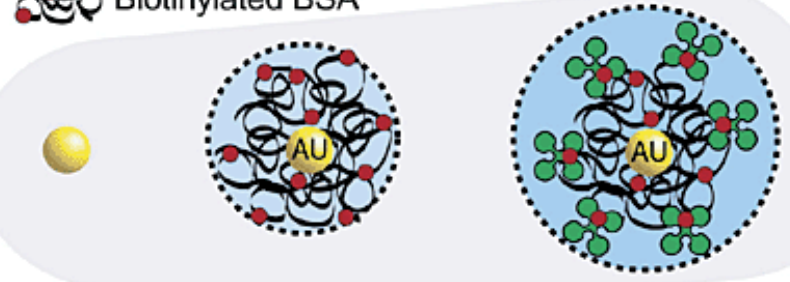

(b)

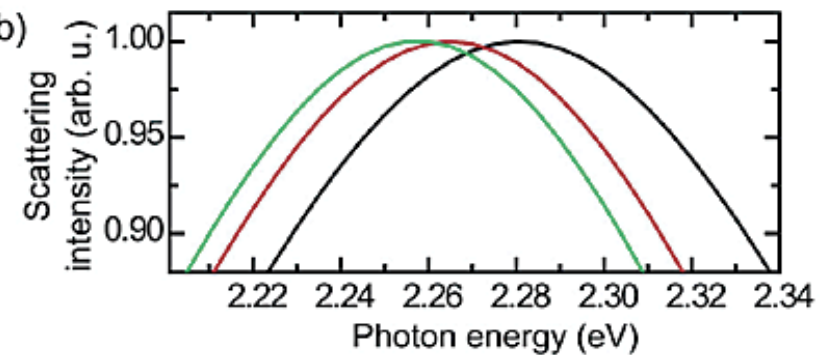

(c)
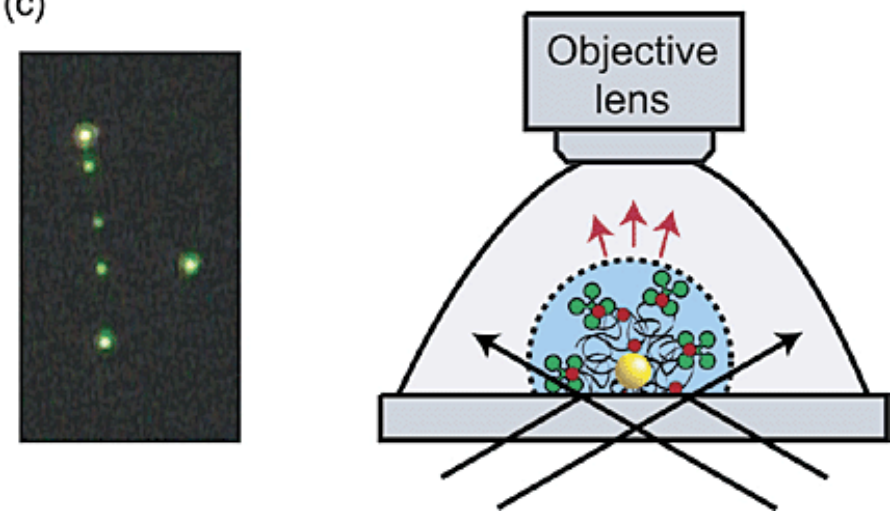

Figure 2.6 Principle and schematic representation of a biosensor based on light scattering from a single gold nanoparticle. (a) Single gold nanoparticles are functionalized with biotinylated BSA which subsequently binds streptavidin. (b) Mie theory calculations for the three different environments shown in (a). (c) Left: true color photograph of a sample of functionalized gold nanoparticles in darkfield illumination. Right: experimental setup facilitating dark-field microscopy of single gold nanoparticles immersed in liquids. Reprinted with permission from Raschke, G. et al. Nano Lett. 3, 935-938 (2003). Copyright 2003 ACS. 


\section{Chapter III}

\section{Synthesis and Characterization of Gold Nanocrystal/DNA Nanostructures}

Reproduced in part with permission from Zanchet, D., Micheel, C.M., Parak, W.J., Gerion, D., and Alivisatos, A.P. Nano Lett. 1, 32-35, Copyright 2001

American Chemical Society and from Zanchet, D., Micheel, C.M., Parak, W.J., Gerion, D., Williams, S.C., and Alivisatos, A.P. J. Phys. Chem. B 106, 1175811763, Copyright 2002 American Chemical Society.

\section{Introduction}

Work on the synthesis and characterization of gold nanocrystal/DNA nanostructures has been ongoing in the Alivisatos group since the mid-1990s.

Over the last decade, progress has been made to improve the method, yields, and characterization of simple nanostructures. My involvement in this aspect of nanostructure research was conducted under the guidance of postdoctoral researcher Daniela Zanchet; together we developed the electrophoretic purification methods described in this chapter. From her, I also learned much of what I know about transmission electron microscopy.

The first major improvement to the synthesis of gold nanocrystal/DNA nanostructures describes the purification of discrete gold nanocrystal/DNA conjugates [11]. Colloidal nanocrystal/DNA conjugates hold the promise of becoming powerful probes for biological diagnostics as well as versatile building blocks for nanotechnology. To work towards realizing this potential, it was 
important to precisely control the number of oligonucleotides bound to the nanocrystal. We demonstrated electrophoretic isolation of 5- and 10-nm gold nanocrystals bearing discrete numbers of single-stranded DNA [7, 18, 86-87].

In the second synthetic improvement [12], these discrete gold nanocrystal/DNA conjugates were used to form so-called 'nanocrystal molecules', such as dimers and trimers of gold nanocrystals connected with DNA oligonucleotides. The use of purified conjugates led to a higher yield of the target structure and gave us better control and understanding of the system.

Since the publication of these two improvements to nanostructure synthesis, a variety of other nanostructures have been synthesized by other Alivisatos group members, sometimes in collaboration with members of the Fréchet group. Trimers and tetramers of gold nanocrystals have been constructed using branched DNA [8], dimers of gold nanocrystals have been constructed using antibody-antigen interactions [13], and semiconductor nanocrystal/gold nanocrystal hybrid nanostructures have also been constructed using DNA interconnects [20].

\section{Discrete Gold Nanocrystal/DNA Conjugate Synthesis and Purification}

Nanocrystal biopolymer conjugates hold great promise both for biological diagnostics, where the nanocrystals can provide unique detection signatures $[18$, 
86-87] and for nanotechnology, where the information content of the biomolecule can be harnessed for spatial patterning of nanocrystals [7, 88-93].

There are many strategies available for bioconjugation of nanocrystals, including attachment to biotin-avidin [18, 94], antigen-antibodies [95], peptides [90, 96], and proteins [96]. Among the many biological polymers that can be coupled to nanocrystals, DNA is of particular interest, because of its inherent programmability. The Watson-Crick base pairing of an oligonucleotide is thermally stable at room temperature when the number of bases is around 12 . Twelve base pairs are approximately $4 \mathrm{~nm}$ in length, or the diameter of a typical nanocrystal, and contain a sufficient number of unique pairs that the strand can be designed to contain detailed instructions for placement of the nanocrystal in a programmed assembly. By 2000 , this remarkable property had already been exploited to create three-dimensional aggregates of nanocrystals [1], to attach nanocrystals to surfaces [97], and to create nanocrystal molecules [7, 10, 88]. Yet to fully utilize nanocrystal/DNA conjugates in creating programmed assemblies, it is first necessary to prepare nanocrystals with a discrete and known number of single-stranded (ss) DNA oligonucleotides attached. In all of my experiments, gold nanocrystals are first conjugated to thiolated, singlestranded DNA and are then hybridized to other DNA or gold nanocrystal/DNA conjugates. Thus, the DNA oligonucleotides will not be labeled as thiolated, single-stranded or double-stranded unless it cannot be easily determined from the context. 
Control over the precise number of oligonucleotides per nanocrystal is essential for diagnostics whenever there is a need to quantify the number of hybridization events, rather than just assess the presence of a particular sequence. Nanocrystals bearing one and only one oligonucleotide strand are particularly important for this purpose. In the fabrication of nano-structured assemblies, it is possible that nanocrystals bearing different numbers of oligonucleotides could serve as the elementary construction units: vertex (4 strands), corners (3 strands), lines (2 strands), terminus ( 1 strand), etc. Finally, it is noted that the nature of interaction between the polyanionic oligonucleotides and the nanocrystals is not well understood but is a very interesting problem in biophysics. The persistence length of ssDNA is about $2 \mathrm{~nm}$ [98], so in some cases DNA strands may be able to wrap around the nanocrystal. Nonetheless, it should be possible to adjust the nanocrystals or the oligonucleotide to promote or suppress this effect [34]. It appears that being able to precisely control the number of bound strands will be very desirable for this purpose.

We demonstrated the electrophoretic isolation of discrete gold nanocrystal/DNA conjugates. Gold was chosen because of its ease of use-the nanocrystals are stable and processable in the high ionic strength buffers needed for manipulation of DNA, and attachment of DNA to the gold via a terminal thiol on the oligonucleotide is straightforward. In the strategy employed, the binding of DNA to the gold is a statistical process. By adjusting the DNA:gold nanocrystal ratio, it 
was possible to control the average number of DNA strands per nanocrystal, but there was always a distribution of oligonucleotides present. Thus, we needed a technique for separation and isolation of conjugates that was very sensitive to the number of bound strands.

Gel electrophoresis is a powerful technique in biology and is widely applied in the separation of DNA of different sizes [99]. In this technique, charged particles migrate in a porous matrix (gel) under an electric field; particle mobility depends on their charge and size. Electrophoresis is also a useful tool in colloidal science, and it has been applied to isolate small gold clusters [100]. It seems then a natural choice to use electrophoresis for characterization and isolation of nanocrystal/DNA conjugates since the binding of DNA to nanocrystals should produce a significant shift in their electrophoretic mobility.

\subsection{Materials and Methods}

These experiments used gold nanocrystals of 5- and 10-nm mean diameter (gold colloids were either purchase from Ted Pella (Redding, CA) or synthesized by the citrate/tannic acid method [41]. Typical size distributions were $15 \%$. The stability of gold colloids was increased by passivation with bis $(\mathrm{p}-$ sulphonatophenyl)phenylphosphine dihydrate, dipotassium salt (Figure 3.1, Strem Chemicals, Newburyport, MA). The modified colloids were precipitated with $\mathrm{NaCl}$, centrifuged, and redissolved to a final concentration of 1-10 $\square \mathrm{M}$.). 
Alkanethiol-modified single-stranded DNA (DNA) 18 to 100 bases (b) in length were purchased from Integrated DNA Technologies (Coralville, IA). DNA sequences: $100 \mathrm{~b}, 5^{\prime}-$

XGCAGTAACGCTATGTGACCGAGAAGGATTCGCATTTGTAGTCTTGAGCCC GCACGAAACCTGGACACCCCTAAGCAACTCCGTA, CAGATGGGAACAGCA3'; 80b: 5'-

XGCAGTAACGCTATGTGACCGAGAAGGATTCGCATTTGTAGTCTTGAGCCC GCACGAAACCTGGACACCCCTAAGCAACTC-3'; 50b, 5'XGCAGTAACGCTATGTGACCGAGAAGGATTCGCATTTGTAGTCTTGAGCCC3'; 18b, 5'-XCAGTCAGGCAGTCAGTCA-3'. X = 5'-thiol modifier.

Nanocrystal/DNA conjugates were prepared by adding a stoichiometric amount of $50 \square \mathrm{M}$ DNA (1, 2, etc. equivalents) to the gold colloid in 0.5X TBE (Tris-borate EDTA buffer), vortexing for a few seconds and adding $\mathrm{NaCl}$ up to a $50 \mathrm{mM}$ concentration. The solutions were vortexed again and incubated at room temperature for $2 \mathrm{~h}$ before the gel was run. The electrophoresis experiments were performed in 2 or $3 \%$ agarose gels at $100 \mathrm{~V}, 6.7 \mathrm{~V} / \mathrm{cm}$, for $1 \mathrm{~h}$. The buffer used was $0.5 \mathrm{X}$ TBE. Gold colloids in this size range have a deep red color 


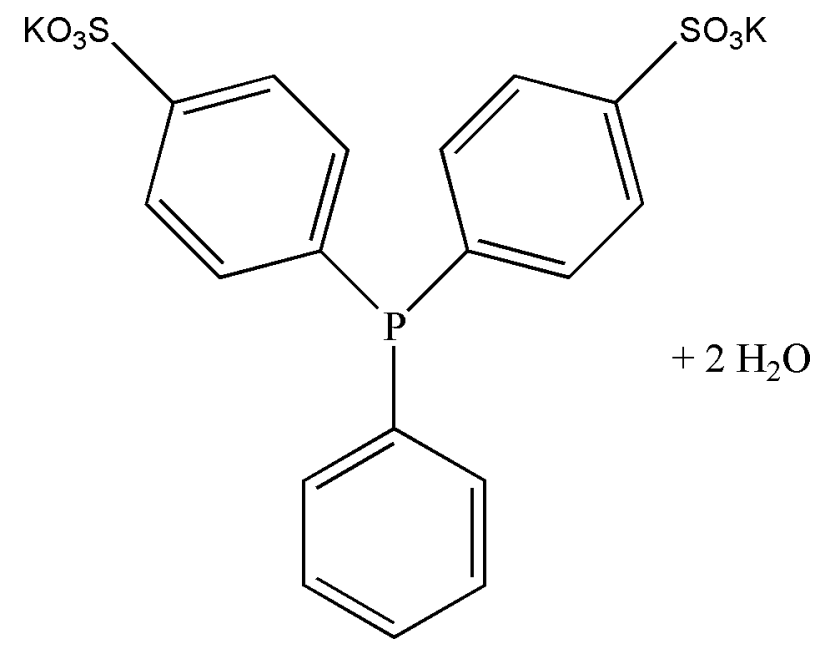

Figure 3.1 Bis-(p-sulfonatophenyl)phenylphosphine dihydrate dipotassium salt 
and can be easily visualized in the gels.

\subsection{Results}

First, the results for 5-nm gold nanocrystals with 100b DNA attached are described, where the most substantial effects are expected. Figure 3.2 shows the results: discrete bands $(1,2,3)$ of lower mobility appear in the same lane when 100b DNA is added to the gold nanocrystals. Doubling the DNA:gold nanocrystal ratio changes the relative intensity of the bands, and in fact new, retarded bands $(4,5)$ appear in the gel. These results clearly indicate a discrete step process that we have assigned as being the number of DNA strands attached to the nanocrystals. Each band, corresponding to a defined number of strands per nanocrystal, can be recovered from the gel using standard techniques [10] and stored for weeks at $4^{\circ} \mathrm{C}$.

Several experiments have been performed that demonstrate that the discrete bands arise from nanocrystals bearing specific numbers of DNA strands. The issue of greatest concern is that the DNA may interact with gold nanocrystals in multiple ways (in addition to the terminal thiol). Non-specific adsorption of DNA lengthwise onto flat gold surfaces is well known [101]. The DNA could bind nonspecifically to the nanocrystals depending on salt concentration or even promote the clustering of the nanocrystals. First, the specific binding of DNA 


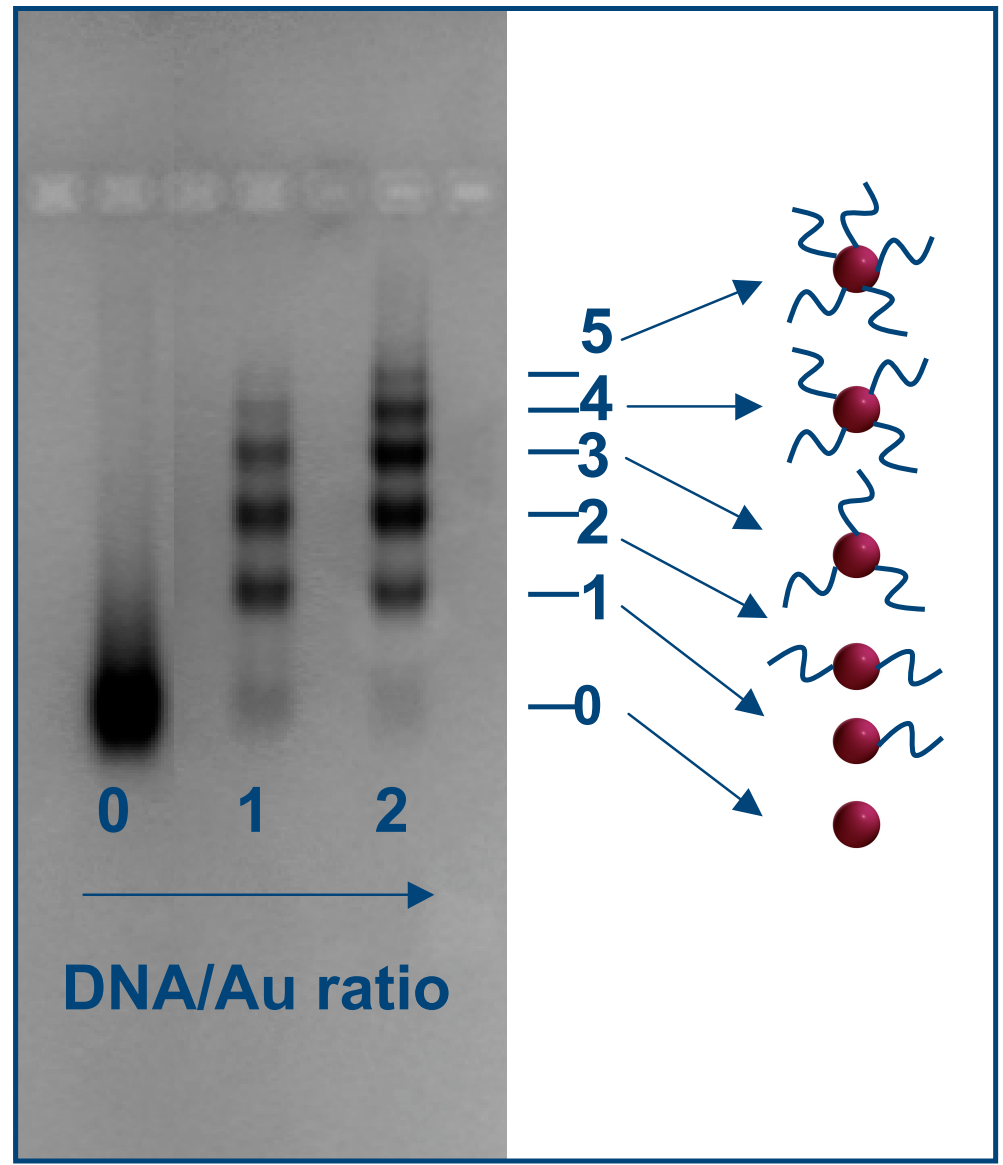

Figure 3.2 Addition of thiolated DNA to gold nanocrystals results in conjugates of gold nanocrystals attached to varying numbers of DNA strands. Electrophoresis of these conjugates yields bands of different conjugates. Gold nanocrystals with no DNA attached are fastest (bottom of gel image), while gold nanocrystals with more DNA attached are slower. Mixing the DNA and gold at a higher DNA:gold ratio yields higher-order conjugates. 
through the thiol group was verified. This was done by adding DNA of the same sequence but without the thiol modifier. As it was clearly demonstrated in this control experiment (Figure 3.3), discrete bands do not appear when 100b DNA is used and there is no significant non-specific binding under these conditions. It should be noted that this effect is time-dependent. If the conjugates are incubated for significantly longer than 2 hours, the bands become diffuse, and it becomes more difficult to use the conjugates to form nanostructures. This is believed to be an effect of the non-specific binding of the DNA to the gold nanocrystals.

Second, it was necessary to prove that the discrete bands do not correspond to nanocrystal clusters (dimers, trimers) mediated by nonspecific DNA interaction. The first experiment entailed recovering the bands $0,1,2$, and 3 (see Figure 3.2) and imaging the nanocrystals in a transmission electron microscope (TEM) (TEM was performed on a Topcon EM002B electron microscope (200 kV) at NCEM, Lawrence National Laboratory (Berkeley, CA) and on a JEM-3010 (300 kV), LME- LNLS (Campinas, Brazil).). For the four bands collected, there was no difference in the nanocrystal distribution on the TEM grid. When these samples were run again in the gel, their mobility remained unchanged. Unless there is some unexpected strong interaction with the carbon substrate on the TEM grid, the change in mobility cannot be assigned to nanocrystal cluster formation. 


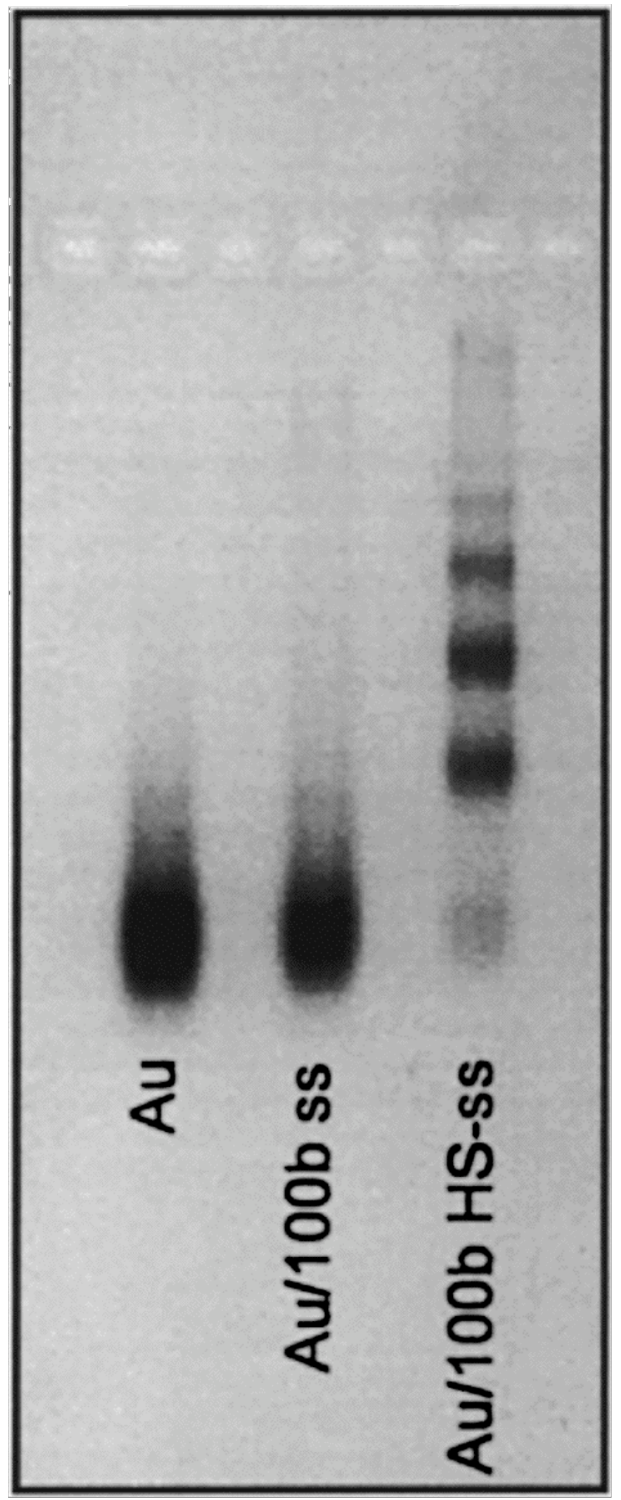

Figure 3.3 Nonthiolated DNA does not bind to the nanocrystals, ruling out nonspecific interaction under these conditions. 
The second experiment involved mixing the recovered conjugate bands with free nanocrystals and then, after several hours, running the mixture through a gel. Again, if the discrete bands corresponded to nanocrystal clustering due to some interaction with DNA, adding free nanocrystals should disrupt this equilibrium and induce the reappearance of the other bands. Figure 3.4a shows that in all cases the lanes corresponded to a superposition of the original conjugate and free nanocrystal bands. In addition, this experiment also proves that the DNA is strongly bound to the gold nanocrystals, since there is no redistribution of number of DNA strands per nanocrystal.

An additional experiment was to study nanocrystals of different sizes. Figure 3.4b shows the bands for 5 -nm gold nanocrystal/100b DNA and 10-nm gold nanocrystal/100b DNA conjugates. Because of the sieving effect of the gel, 10nm nanocrystals migrate less than 5-nm nanocrystals, and the shift of 10-nm gold nanocrystal/100b DNA conjugates is smaller than the 5-nm gold nanocrystal/100b DNA ones. When 5- and 10-nm nanocrystals are mixed with subsequent addition of DNA, it should produce a superposition of the two sets of bands. In contrast, if nanocrystal clusters were formed all combinations would be possible (5-5, 5-10, and 10-10 nm dimers; 5-5-5, 5-5-10, and other trimers; etc.), and a much larger number of bands would be superposed, probably resulting in a single spread band. In Figure 3.4c, the first two lanes correspond to the control samples of 5- and 10-nm nanocrystals, prepared in the same conditions as in the mixture (gold nanocrystal concentration and gold nanocrystal:DNA ratio), while 


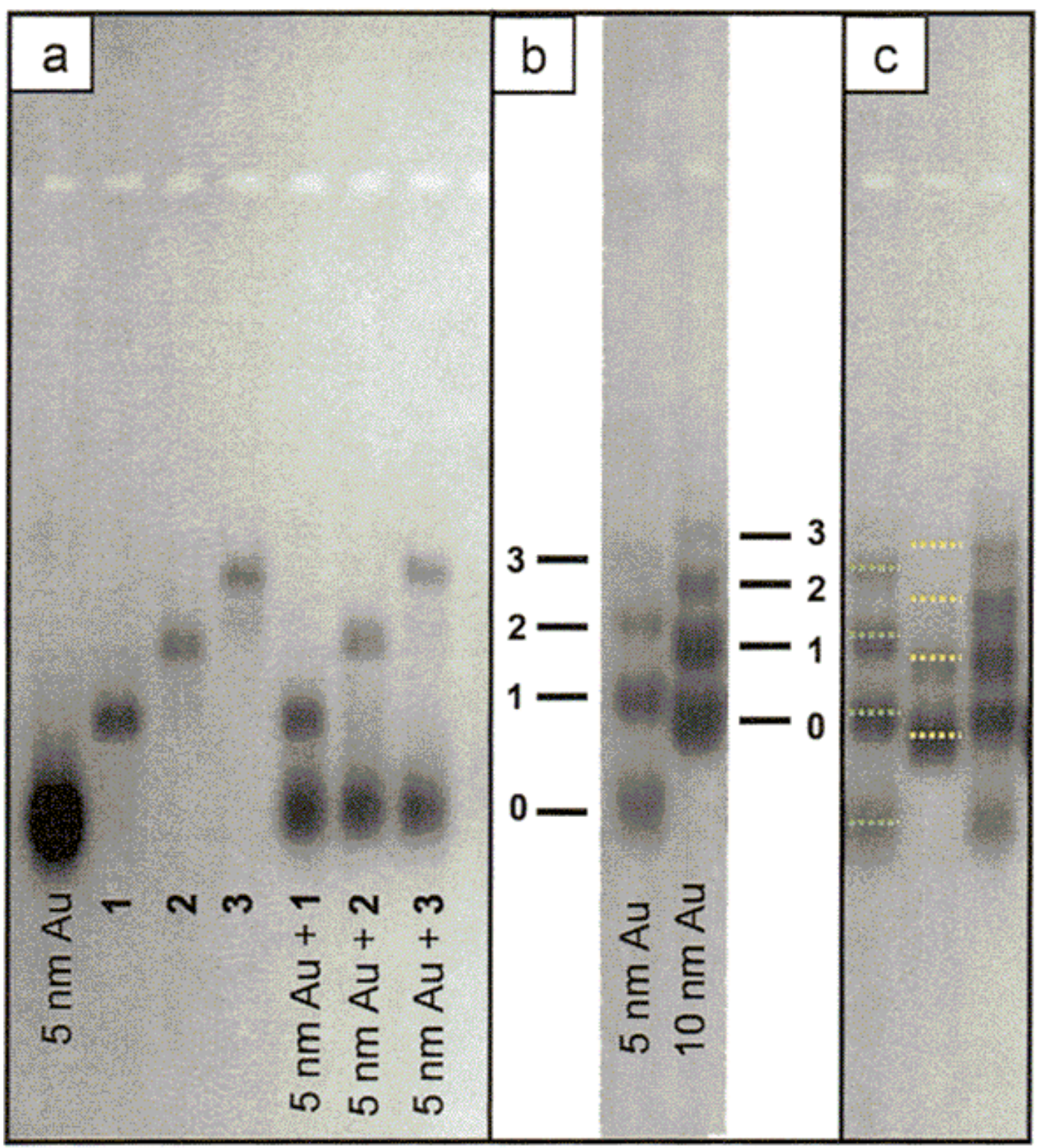

Figure 3.4 (a) 5-nm gold nanocrystal sample and recovered 5-nm gold nanocrystal/100b DNA conjugate bands $(1,2,3)$. Mixing each recovered band with 5-nm gold colloid $(\mathrm{Au}+1, \mathrm{Au}+2, \mathrm{Au}+3)$ generates superposed bands. (b) Gold nanocrystal/100b DNA conjugates for 5- and 10-nm nanocrystals. (c) Adding DNA to a mixed solution of 5- and 10-nm nanocrystals (last lane) leads to a superposition of 5-nm gold nanocrystal/100b DNA (first lane) and 10-nm gold nanocrystal/100b DNA (second lane) bands nanocrystal/DNA. 
the third lane corresponds to the mixed solution. It is clear that the third lane keeps the discrete character and that all the bands can be assigned to either 5nm gold nanocrystal/100b DNA or 10-nm gold nanocrystal/100b DNA conjugates. The mobility pattern also suggests that the DNA preferentially binds to the 10-nm nanocrystals when both 5- and 10-nm are present in the same solution, but further experiments need to be done to understand this effect.

The success of the electrophoretic isolation of nanocrystal/DNA conjugates depends on several factors. First, the nanocrystal sample must be homogeneous in both charge and particle size distributions in order to obtain narrow bands. These two factors can be monitored and in some ways regulated. In the case of homogeneous samples, the separation is limited by intrinsic mobility of the nanocrystals and DNA. The mobility of $100 \mathrm{~b}$ DNA and 5-nm nanocrystals is similar in our conditions so the conjugation of the two leads to a significant shift in their mobility. For shorter DNA strands, which migrate faster in the gel, the effect on the nanocrystal mobility should be smaller. Figure $3.5 a$ emphasizes the difference in the mobility of the first nanocrystal/DNA conjugate (one strand) for 50b, 80b, and 100b DNAs. We note that for DNA sizes below $50 \mathrm{~b}$, the 0 and 1 bands will be barely resolved in these conditions and it will be difficult to isolate the discrete nanocrystal/DNA conjugates. Increasing the gel concentration or running time will not significantly improve the separation due to the spread of both bands and, in this case, a better nanocrystal size/charge distribution is probably required. Nonetheless, we could still use electrophoresis 


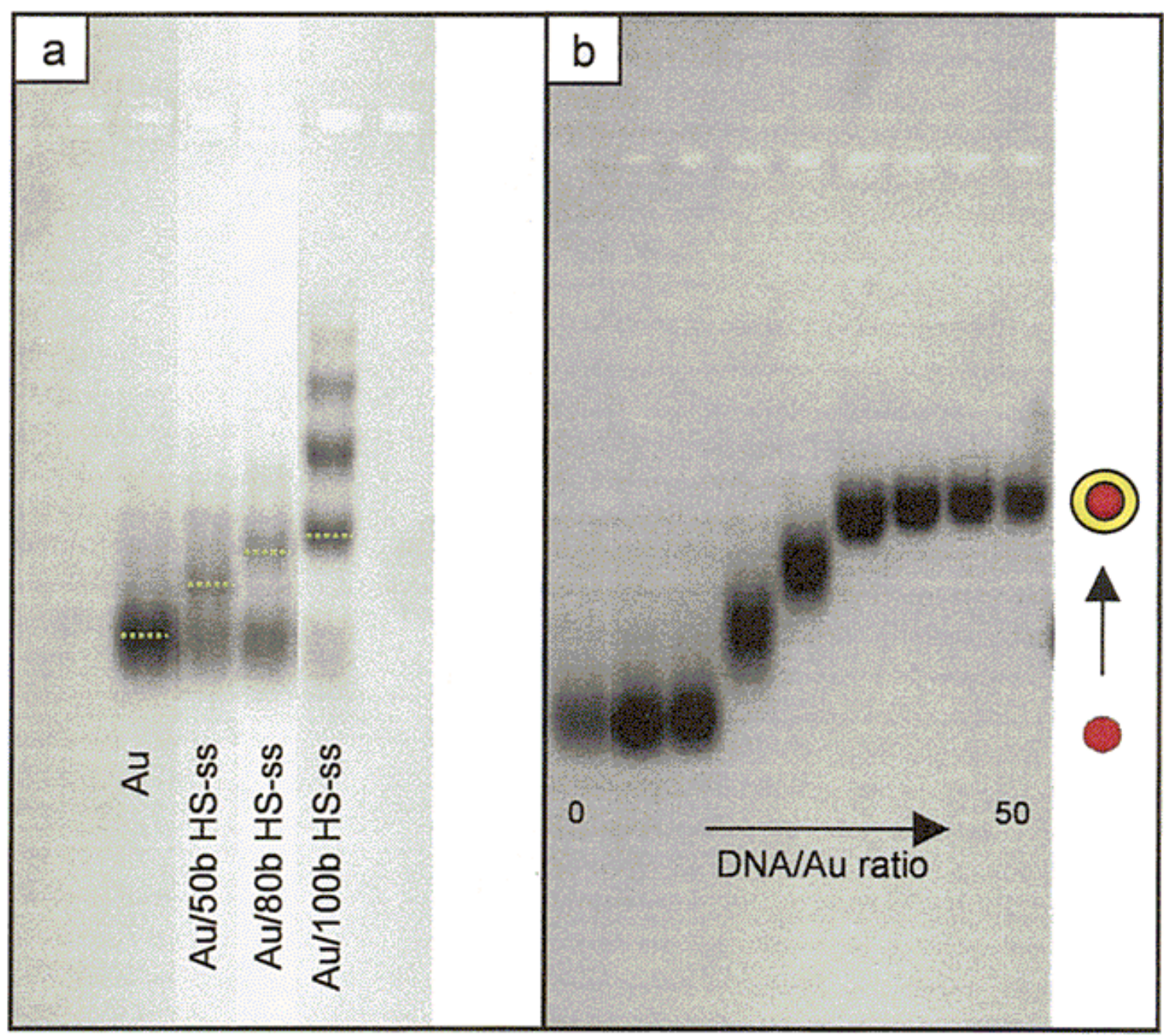

Figure 3.5 (a) 5-nm gold nanocrystal sample and gold nanocrystal/DNA conjugates $(\cdots)$ of different DNA lengths $(50 \mathrm{~b}, 80 \mathrm{~b}$, and $100 \mathrm{~b} ; 3 \%$ gel). (b) Electrophoretic mobility of 5-nm gold nanocrystal/18b DNA (2\% gel). The DNA:gold nanocrystal molar ratios from the left to the right are 0 (pure colloid), 1 , $2,5,10,20,30,40$ and 50 ; these values reflect the increase of the number of DNA strands per nanocrystals, but they do not correspond to the actual values. 
for short DNA conjugates, for example, by hybridizing the short strand with a long one, then isolating the conjugates and finally releasing the long DNA.

Gel electrophoresis can be also exploited to determine the average number of strands per nanocrystal, for these short DNA strands. Figure $3.5 \mathrm{~b}$ shows the results for 5-nm gold nanocrystal/18b DNA conjugates. The first lane corresponds to the 5-nm unconjugated nanocrystals, and the second and third lanes correspond to samples prepared with DNA:gold nanocrystal molar ratios of 1 and 2. While the nanocrystals run in well-defined bands, the addition of small amounts of 18b DNA does not affect the overall mobility of the nanocrystals. Nevertheless, by increasing the DNA:gold nanocrystal ratio to 5 or 10 , multiple strands attach to the nanocrystals, producing a decrease in the nanocrystal mobility. We achieve a saturation limit around 20 DNA:gold nanocrystal molar ratio, above which the nanocrystal mobility does not change significantly. However, we note that the later bands are narrower, which indicates a more homogeneous charge distribution due to a more complete coverage with DNA. Decreasing the gel concentration would help distinguish the bands above 20 strands per nanocrystal. Unfortunately, we could not directly correlate the DNA:gold nanocrystal molar ratio in solution with the actual number of DNA strands per nanocrystal. We could, however, infer that in lane 5 there is more DNA per nanocrystal than in lanes 2 and 3. 


\subsection{Conclusions}

In summary, we were able to isolate discrete nanocrystal/DNA conjugates by gel electrophoresis. Using 5- and 10-nm gold nanocrystals, we demonstrated that the shift in the nanocrystal mobility due to the DNA attachment is a very powerful probe used to study and isolate several conjugates; this method can also be extended to different systems. Nanocrystals with a well-defined number of DNA strands can be seen as new building blocks in nanotechnology as well as important probes for quantitative detection analysis.

\section{Use of Discrete Gold Nanocrystal/DNA Conjugates in Nanostructure Formation}

In the area of biomolecule-assisted nanocrystal assembly, our work has centered on the creation of nanocrystal molecules made from gold nanocrystals connected with DNA. There are several advantages to this approach. First, the structures created are discrete: the structure resulting from a given set of starting materials is predictable and can be reproduced precisely and accurately. In addition, it is no more difficult to synthesize billions of copies of a desired structure than it is to create a single copy; this is potentially a very powerful tool. Finally, the structures are easily controlled because of the programmability of the scaffolding material-DNA. Using DNA, we can control the number and size of nanocrystals linked, the distance between nanocrystals, the dimensionality, and the flexibility 
of the structures. Gold nanocrystals were chosen as a model system because of the homogeneity of size and shape and their easy manipulation in a single solvent with straightforward chemistry. However, it is important to note that the protocol is general and can be extended to several other systems, such as semiconductor nanocrystals.

Two previous papers from our group presented the synthesis of dimers and trimers of gold nanocrystals connected with DNA $[7,10]$. In these papers, complementary conjugates (nanocrystals attached to DNAs of complementary sequences) were combined to form dimers and trimers by DNA hybridization. Although a 1:1 gold nanocrystal/DNA ratio was used to produce nanocrystals bearing just one DNA, the binding of the DNA to the nanocrystal is a statistical process and the final number of DNAs per nanocrystal was not homogeneous. Usually, free nanocrystals, dimers, and trimers were present in a final mixture if dimers were the target structure, for example, which had to be purified afterward. While this approach succeeded in generating nanocrystal molecules, it could not be extended to more complex systems. In addition, the low yield achieved $(<30 \%)$ for the generated structures was a limiting factor for the system characterization.

In the current work, we used highly purified gold nanocrystal/DNA conjugates: that is, nanocrystals bearing a specific number of DNA (one to five) [11] as building blocks to form dimers and trimers. This approach produced the target 
structure in much higher yield, and it allowed better control of the system and improved understanding of the DNA attachment to the nanocrystal. Several issues have been addressed, and the techniques developed improved all of our subsequent related research. By using gel electrophoresis, we studied the mobility of gold nanocrystal/DNA hybrid materials, pointing out the critical role of the surface charge of the nanocrystals. Besides dimers and trimers, intermediate building blocks were also identified in the gel, such as dimers of nanocrystals bearing one extra DNA. The high yield in the final structures allowed better characterization by transmission electron microscopy (TEM) and development of a computer program to generate pair distribution functions from TEM images, which will be discussed in a later chapter.

\subsection{Materials and Methods}

Gold colloids with mean diameters of 5- and 10-nm were either synthesized by the citrate/tannic acid method [41] or purchased (Ted Pella). Typical size distributions were about $15 \%$. Nanocrystals were coated with a phosphine ligand (bis(p-sulfonatophenyl)phenylphosphine dihydrate, dipotassium salt (Strem Chemicals) as described previously [10-11] and concentrated up to micromolar range (Figure 3.1). The phosphine coating gives a net negative charge on the nanocrystal surface, giving stability in high concentrations and in buffer conditions. Polyacrylamide gel electrophoresis (PAGE)-purified thiol-modified DNAs with 50,80 , and 100 bases (b) (DNA sequences: 50b, 5'- 
XGCAGTAACGCTATGTGACCGAGAAGGATTCGCATTTGTAGTCTTGAGCCC3'; 80b, 5'-

XGCAGTAACGCTATGTGACCGAGAAGGATTCGCATTTGTAGTCTTGAGCCC GCACGAAACCTGGACACCCCTAAGCAACTC-3'; 100b, 5'-

XGCAGTAACGCTATGTGACCGAGAAGGATTCGCATTTGTAGTCTTGAGCCC GCACGAAACCTGGACACCCCTAAGCAACTCCGTATCAGATGGGAACAGCA3'; X = 5'-thiol modifier) were purchased from Integrated DNA Technologies (Coralville, IA; unless otherwise mentioned, the abbreviation DNA will stand for thiolated ssDNA in this work).

The generation of discrete gold nanocrystal/DNA conjugates was described in the previous section. The mean size of gold nanocrystals is either 5- or 10-nm (note that small variation from batch to batch was found), and the DNA length is $50 \mathrm{~b}, 80 \mathrm{~b}$, or $100 \mathrm{~b}$. In this way, the abbreviation $1 / 5 \mathrm{~nm}$ gold nanocrystal/100b DNA corresponds to 5-nm gold nanocrystals bearing 1 DNA of $100 \mathrm{~b}$ in length. The first number, then, refers to the number of DNA attached to the nanocrystal.

Dimers and trimers of nanocrystals were formed by mixing stoichiometric amounts of the corresponding building block conjugates. For dimers, the first conjugates (one gold nanocrystal attached to one DNA strand) with complementary DNAs are mixed in equimolar amounts (final concentration $3 \square \mathrm{M}$ ) and incubated overnight in $50 \mathrm{mM} \mathrm{NaCl}$ or $0.5 \mathrm{X}$ TBE to allow the DNA hybridization. A 3\% agarose gel is used to confirm the dimer formation. A 
similar protocol was used to form trimers of nanocrystals, but a 2:1 ratio of the first and second complementary conjugates was mixed.

TEM studies were performed in an FEI Tecnai 12 TEM, $100 \mathrm{kV}$, at the Electron Microscope Laboratory, UC Berkeley, Berkeley, CA. Formvar-coated grids were first treated with bacitracin, $500 \mathrm{~g} / \mathrm{mL}$ (Sigma), for $30 \mathrm{~s}$, and then gold nanocrystal grouping samples (100-500 nM) were dropped and wicked off after $30 \mathrm{~s}$. For pair distribution analysis, grids were also washed with water for $30 \mathrm{~s}$ both after bacitracin coating and after gold sample coating.

\subsection{Results}

Nanocrystal molecules are formed through interconnection of nanocrystals by a linker. DNA is one of the best linker candidates because of its remarkable properties such as specificity, recognition, and programmability. By programming the DNA sequence on a nanocrystal's surface, one can specifically address the nanocrystal to a binding site.

We used stoichiometric amounts of purified conjugates to generate nanocrystal molecules. The nanocrystal molecules were characterized using gel electrophoresis as well as TEM. It should be noted that UV/vis spectroscopy is not a useful characterization technique for the nanocrystal molecules because the gold nanocrystals connected with DNA are not close enough to produce a 
significant shift in the plasmon peak of the gold. Figure 3.6 shows the typical procedure for obtaining homodimers of 5 -nm nanocrystals using $1 / 5 \mathrm{~nm} / 100 \mathrm{~b}$ DNA complementary conjugates. The first gel (Figure 3.6a) shows the bands corresponding to discrete conjugates of 5-nm gold nanocrystal/100b DNA (A). Each conjugate was then recovered from the gel, the typical yield being up to $30 \%$ for the most intense band. The conjugation yield can be adjusted to some extent by changing the gold nanocrystal/DNA ratio (increasing the DNA amount increases the higher-order conjugate yield). In parallel, the same procedure was performed using 5-nm gold nanocrystals and complementary 100b DNA ( $\left.A^{\prime}\right)$. The dimers were generated by mixing equimolar amounts of $A$ and $A$ ' first conjugates and incubating overnight. Figure $3.6 \mathrm{~b}$ shows the final result: the first three lanes correspond to the reference samples, that is, 5 -nm gold nanocrystals, $1 / 5 \mathrm{~nm} / 100 \mathrm{~b}$ DNA (A), and complementary $1 / 5 \mathrm{~nm} / 100 \mathrm{~b}$ DNA ( $\left.\mathrm{A}^{\prime}\right)$, and the fourth lane is the dimer sample. The dimer band can be easily identified in the fourth lane, as a retarded band, and it corresponds to more than $80 \%$ of the species in the sample. The slower mobility of dimers related to the former conjugates is explained by their larger size (sieving effect).

Extending this procedure to heterodimers of 5 - and $10-\mathrm{nm}$ nanocrystals $(5 / 10)$ and homodimers of 10 -nm nanocrystals $(10 / 10)$ is straightforward. Figure 3.7 shows the resulting gel and the TEM images are presented in Figure 3.8. The first four lanes are the reference samples, and the following three lanes correspond to the $5 / 5,5 / 10$, and $10 / 10$ dimers (left to the right). As expected, the 


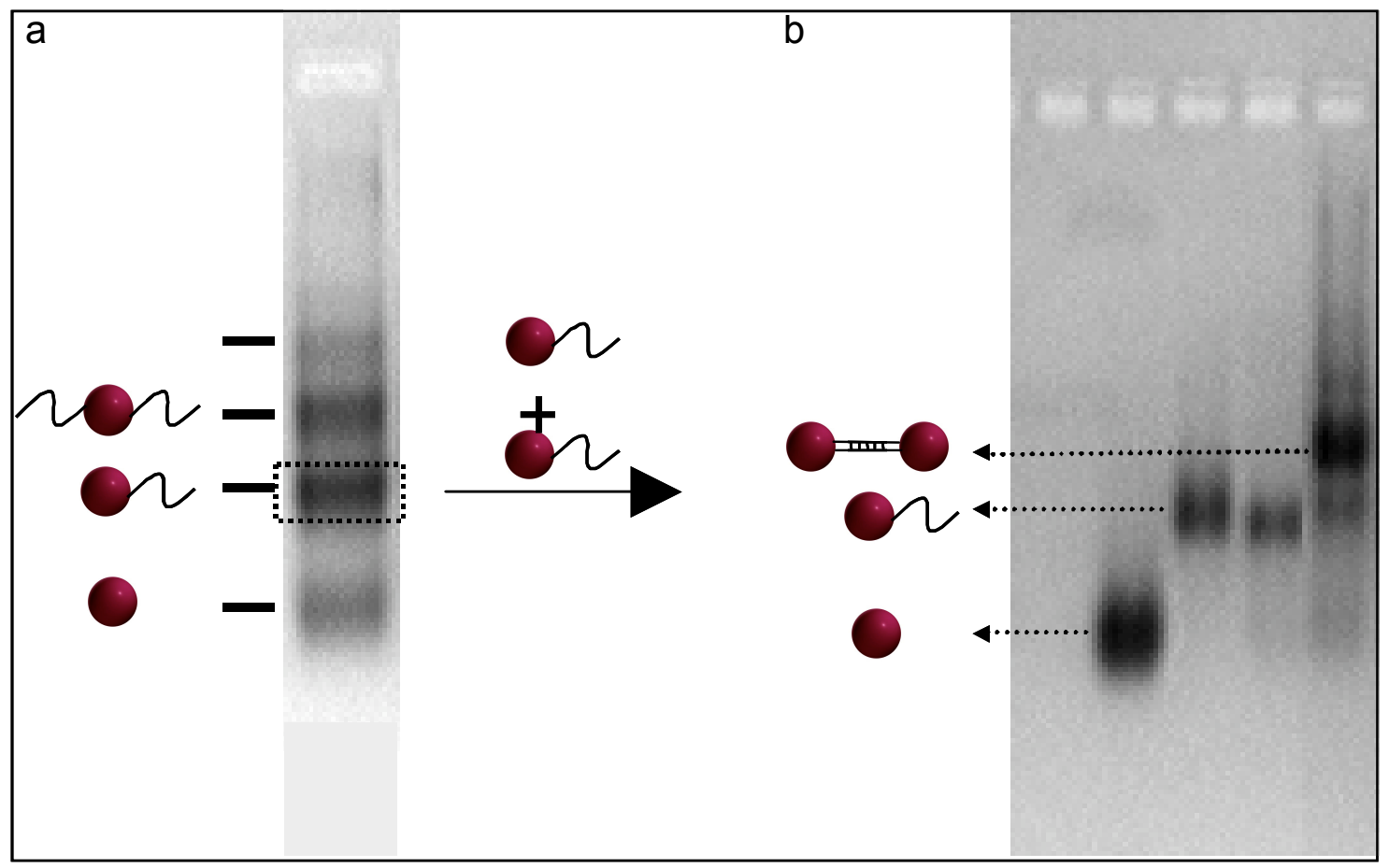

Figure 3.6 A $3 \%$ agarose gel (a) showing the $5 \mathrm{~nm} / 100 \mathrm{~b}$ DNA conjugates. To form $5 / 5$ dimers, the first conjugate is collected (dashed square), mixed with an equimolar amount of a complementary conjugate, and incubated overnight in 50 $\mathrm{mM} \mathrm{NaCl}$. Panel b shows a 3\% agarose gel illustrating the dimer formation; the first three lanes (left to right) are the references, $5 \mathrm{~nm}, 1 / 5 \mathrm{~nm} / 100 \mathrm{~b}$ DNA, complementary $1 / 5 \mathrm{~nm} / 100 \mathrm{~b} \mathrm{DNA}$, and the last lane is the dimer sample. The dimer band is slower than the $1 / 5 \mathrm{~nm} / 100 \mathrm{~b}$ DNA because of the sieving effect. 


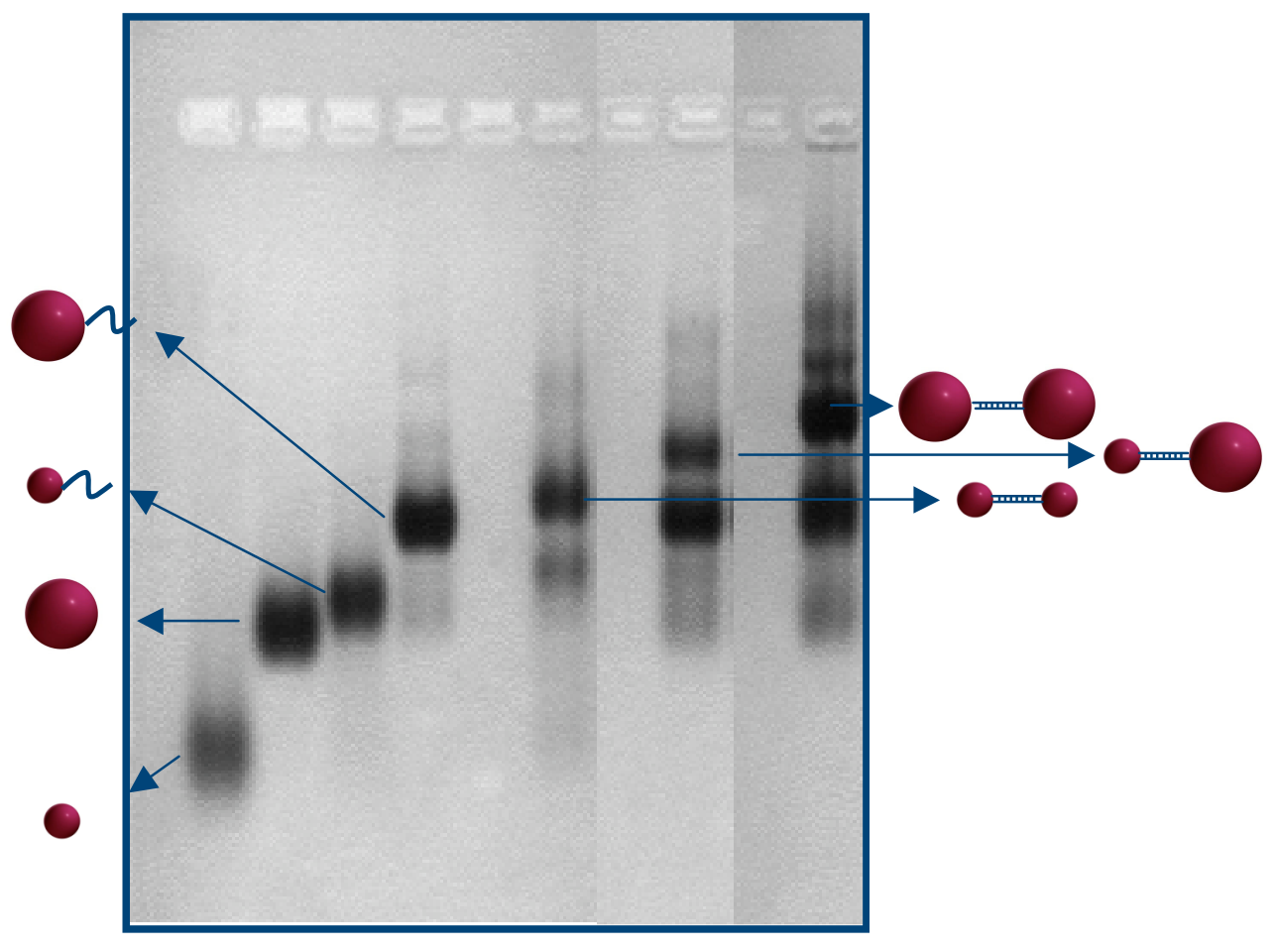

Figure 3.7 Formation of different dimers: 5/5, 5/10, and 10/10. From left to right are shown the reference lanes, $5 \mathrm{~nm}, 10 \mathrm{~nm}, 1 / 5 \mathrm{~nm} / 100 \mathrm{bssDNA}$, and 1/10 nm/100bssDNA; then, the dimer lanes show $5 / 5,5 / 10$, and 10/10 dimers. 


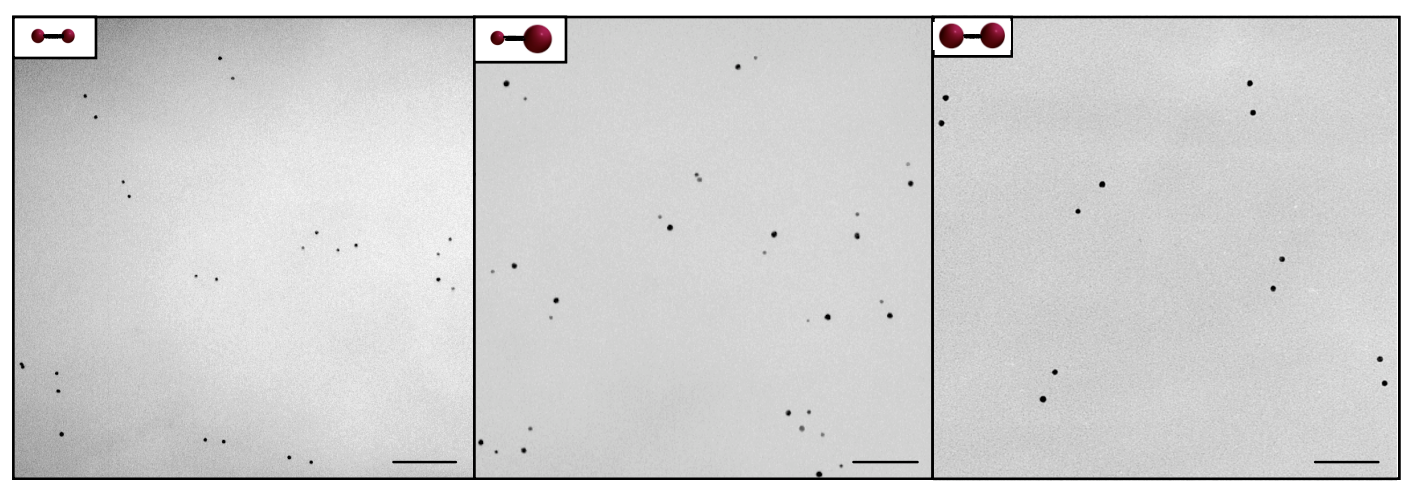

Figure 3.8 TEM images of the dimer bands: from the left to right, $5 / 5,5 / 10$, and $10 / 10$ dimers. The scale bar is $100 \mathrm{~nm}$. 
dimer mobility depends on nanocrystal sizes. The other bands found in lanes 5-7 correspond to uncombined conjugates.

This methodology can also be easily extended to form more complex structures, such as trimers. In this case, the intermediary structures were also probed, such as dimers of nanocrystals bearing one extra DNA (dimer building block). This is important information for determining the usefulness of electrophoresis in this kind of study. Two different heterotrimers were built: 5/10/5 and 10/5/10. The first four lanes in the gel of Figure 3.9 are the references: $5 \mathrm{~nm}, 10 \mathrm{~nm}, 1 / 5$ $\mathrm{nm} / 100 \mathrm{~b}$ DNA, and 2/10 nm/100b DNA. Lanes 5-7 correspond to the combination of $1 / 5 \mathrm{~nm} / 100 \mathrm{~b}$ DNA and $2 / 10 \mathrm{~nm} / 100 \mathrm{~b}$ DNA in different ratios: 1:1, $2: 1$, and 5:1. The combination of the conjugates in a 1:1 ratio (lane 5) led to the appearance of a more retarded band, corresponding to the $5 / 10$ dimer building block. Doubling the amount of $1 / 5 \mathrm{~nm} / 100 \mathrm{~b}$ DNA increased the intensity of this retarded band. Finally, when a 5:1 ratio was used, an additional retarded band was visible, corresponding to the trimer $5 / 10 / 5$. It is worth pointing out that in this experiment the $5 / 10$ dimer building block and the 5/10/5 trimer were not well separated, probably because of the small difference in the final structure size.

On the other hand, the last lane corresponds to the 10/5/10 trimer, and we can see that its separation from the $10 / 5$ dimer building block is clearer, because of a greater difference in size (a larger nanocrystal, $10 \mathrm{~nm}$ instead of $5 \mathrm{~nm}$, is added to the corresponding dimer building block). Figure $3.9 \mathrm{~b}$ shows the TEM image 


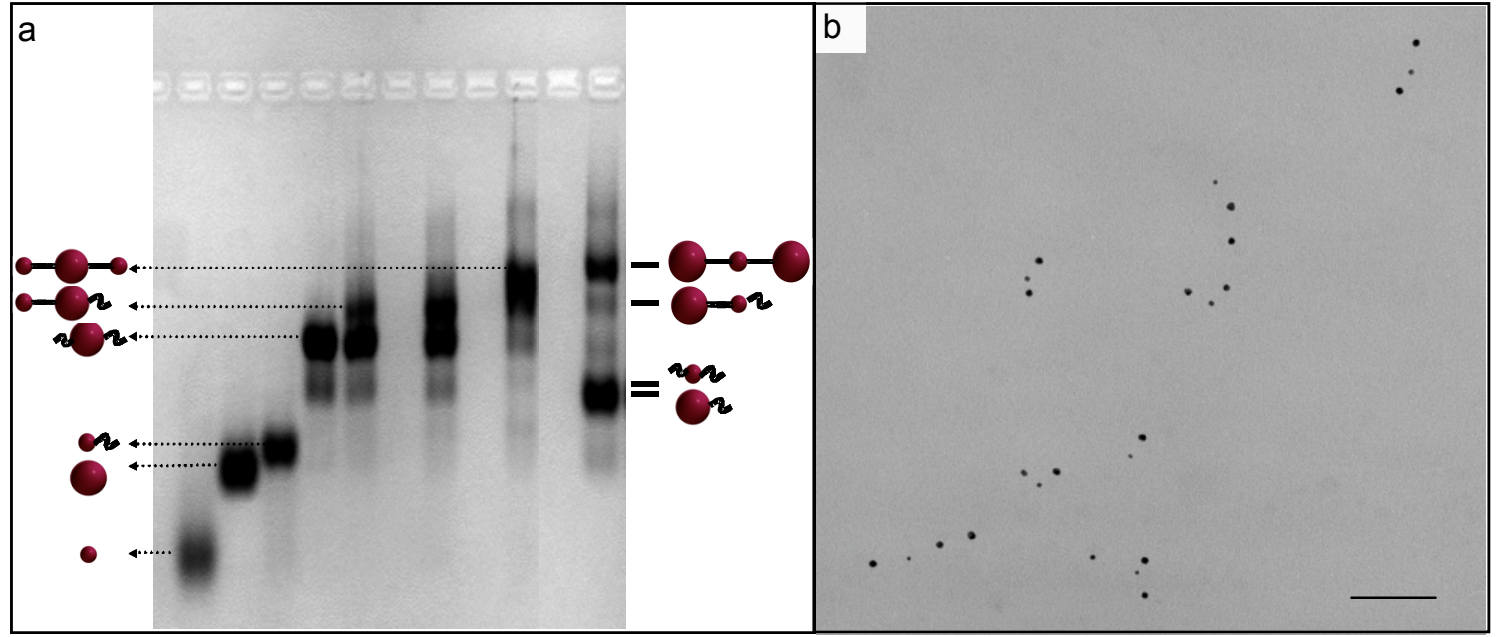

Figure 3.9 Trimer and dimer (a) building blocks. Reference lanes (from left to right) $1-4$ correspond to $5 \mathrm{~nm}, 10 \mathrm{~nm}, 1 / 5 \mathrm{~nm} / 100 \mathrm{bssDNA}$, and 2/10 nm/100bssDNA. Lanes 5-7 correspond to the 5/10/5 trimer formation: (5) 1:1 mixture of $1 / 5 \mathrm{~nm} / 100 \mathrm{bssDNA}$ and 2/10 nm/100bssDNA; (6) 2:1 ratio; (7) 5:1 ratio. The addition of $1 / 5 \mathrm{~nm} / 100 \mathrm{bssDNA}$ to the $2 / 10 \mathrm{~nm} / 100 \mathrm{bssDNA}$ leads to the formation of the corresponding dimer building block (5/10 dimer with an extra ssDNA). Increasing the $1 / 5 \mathrm{~nm} / 100 \mathrm{bssDNA}$ ratio generated the trimer $5 / 10 / 5$. Lane 8 corresponds to the $10 / 5 / 10$ trimer and related building units. Panel $b$ shows the TEM image of $10 / 5 / 10$ trimers; scale bar is $100 \mathrm{~nm}$. 
of the $10 / 5 / 10$ trimers. Although we cannot yet fully explain the reasons for the incomplete hybridization of the conjugates, the fact that we are dealing initially with purified samples is a prerequisite to overcoming this issue. Possible reasons could be related to the annealing process, DNA angle distribution, and the purification process.

\subsection{Conclusions}

The improvement in our ability to make and characterize gold nanocrystal/DNA structures suggests several routes for future work. One of the current challenges is the generation of discrete structures formed by more than two or three nanocrystals, and the use of conjugates makes it more realistic to pursue this. Toward this goal, mobility studies were conducted [14-15], as well as the development of the pair distribution function analysis [24]; this will allow better evaluation of complex structures.

The combination of different conjugates in various proportions can lead to the formation of a great variety of nanocrystal assemblies, from two (dimers) to numerous nanocrystals interconnected in a specific way. The methodology is general and can be extended to other kinds of systems (nanocrystals and biomolecules), providing a vast library of building blocks. We expect that this will allow us soon to study the evolution of properties (optical, electrical) as a function 
of the number and distribution of interconnected nanocrystals in the same way that we now study the properties of assemblies of atoms.

\section{Conclusions}

With this work, it became possible to reliably purify and characterize conjugates and nanostructures using gel electrophoresis. This was essential for further development in the synthesis of discrete nanocrystal/biomolecule nanostructures. Electrophoresis has become an indispensable tool in the synthesis and characterization of discrete nanocrystal/biomolecule structures. Finally, this work led to further research into the electrophoretic properties of discrete nanostructures (see Chapter IV) as well as further development of the methods used to characterize these structures (see Chapter V). 


\section{Chapter IV}

\section{Electrophoretic Mobility Studies of Gold Nanocrystal/DNA Conjugates}

Reproduced in part from Zanchet, D., Micheel, C.M., Parak, W.J., Gerion, D., Williams, S.C., and Alivisatos, A.P. J. Phys. Chem. B 106, 11758-11763, Copyright 2002 American Chemical Society.

\section{Introduction}

Nanocrystal/DNA conjugates are versatile building blocks that can be used to generate complex nanosystems. While the physical properties are mainly determined by the nanocrystal, the binding and addressability attributes are given by the sequence of the DNA. The possibility of creating a library of nanoscale building blocks by combining different nanocrystals with different numbers and sequences of DNA is then a challenging and tempting task. To pursue this aim, I explored the electrophoretic mobility of nanocrystals, DNA, and their conjugates to better understand the primary method of separation and purification for these structures. While part of this work was published in reference 12 , I was the primary researcher working on this topic.

As has already been described, the work of learning how to construct discrete inorganic nanocrystal/biomolecule nanostructures is being done in the context of a new field of research, which is at the boundary of molecular biology and nanoscience. Each parent field contains materials with unique properties that 
can be harnessed to solve problems in the other field. For example, the information-storing capability of DNA can be used to pattern nanocrystals, allowing nanoscientists to study a variety of nanocrystal properties $[12,15-17$, 20]. Conversely, the tunability of semiconductor nanocrystals can be used in fluorescence cell labeling experiments $[18,86]$. In order to realize the full potential of materials from each field, we need to understand the interactions between nanocrystals such as gold nanocrystals, and biomolecules such as DNA.

Work in the Alivisatos group has made progress in each direction between molecular biology and nanoscience. First, semiconductor nanocrystals with different fluorescence colors have been used to label cells [18]. Nanocrystals can be directed to specific cellular components by altering the surface chemistry of the nanocrystals. In order to increase the number of colors it is possible to use in the experiments, more ways to address nanocrystals to cellular components need to be developed. To do this, use of more specific interactions is needed. This is one reason I began to try to understand the physical characteristics of nanocrystal/biomolecule conjugate systems.

Another success of the Alivisatos group has been to create nanostructures that use DNA as the scaffolding material $[7-8,10-12,20]$. As was discussed in the previous chapter, the development of electrophoresis for purification and characterization helped to enable the construction of more complex gold 
nanocrystal/DNA nanostructures. Nonetheless, in order to create more complex nanostructures, better methods for characterizing our starting materials were needed. Electrophoresis as a characterization method was difficult to quantify, and yields were frequently less than optimal. In order to address these problems, and to maximize the benefit of electrophoresis as a purification and characterization method, work was undertaken to understand the hydrodynamic properties of hybrid inorganic nanocrystal/biomolecule systems in agarose gels.

\section{Background}

The components of these experiments have several features that are worth noting. Gold nanocrystals and DNA have characteristics that make them good choices for studying the interactions between them in conjugates. Both are easily synthesized and are water-soluble. Their size and charge densities are the same range, though their shape and flexibility are quite different. For example, gold nanocrystals are rigid spheres, while DNA molecules are flexible random coils that inhabit spheres with a length-dependent effective radius

(Figure 4.1). This flexibility makes the electrophoretic properties of a DNA strand and a gold nanocrystal different in the fibrous network of a gel, even when they have the same effective radius.

These are the characteristics that had initially led to the choice of electrophoresis as a method to purify and characterize nanocrystal/biomolecule nanostructures. 


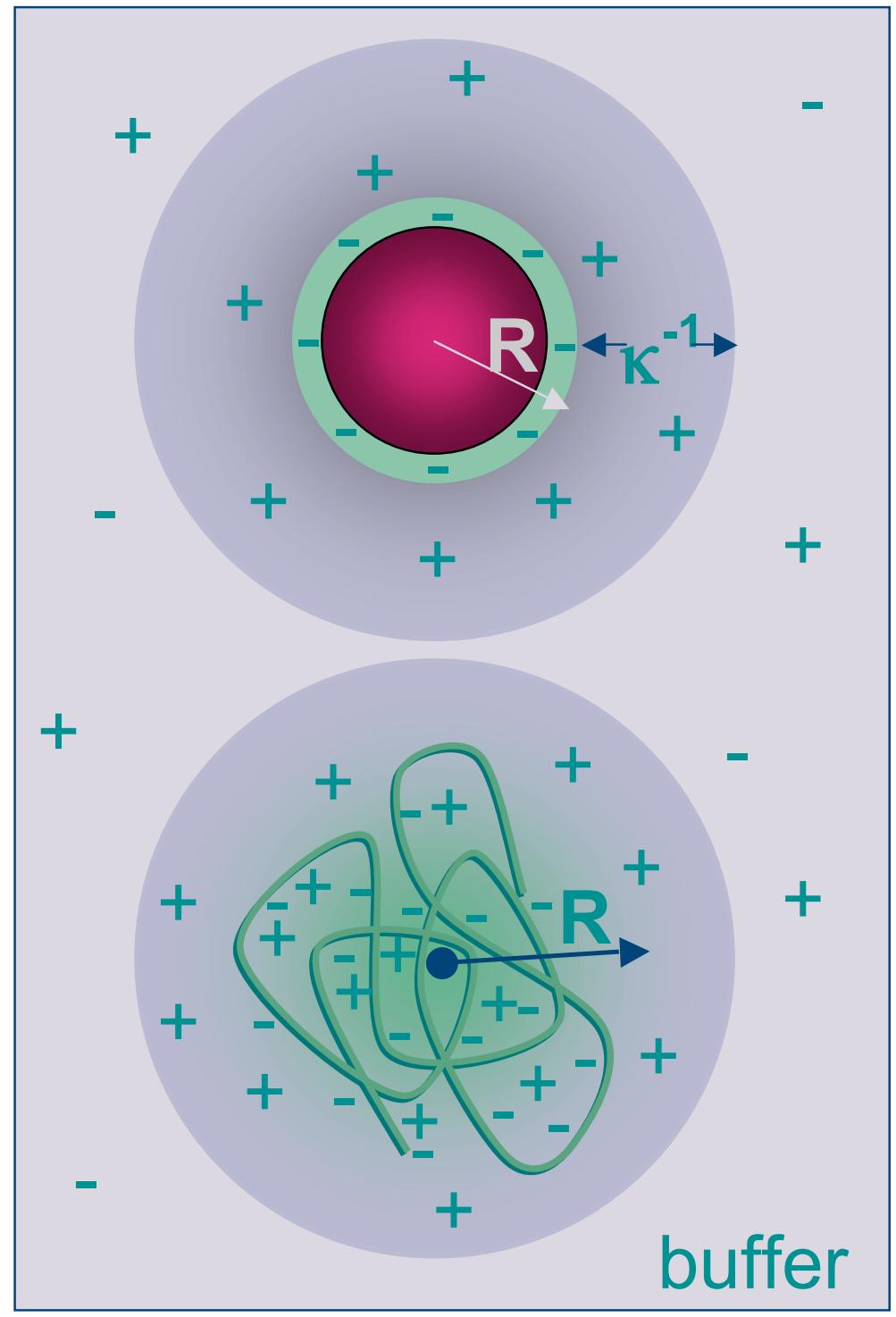

Figure 4.1 Depiction of a gold nanocrystal (top) and DNA strand (bottom) in a buffer solution. 
Electrophoresis is a versatile and ubiquitous tool in biology. It is used for a wide variety of applications, including size and shape determination of biological molecules, determination of charge characteristics-for example, $\mathrm{pH}$ dependence—as well as binding kinetics experiments—such as DNA-binding protein interactions—genetic sequencing, and simple purification. The versatility of the technique made it a convenient and useful technique for us to study the properties of the gold nanocrystal/DNA conjugates. The principles of electrophoresis are relatively simple. Charged particles migrate through a gel under the influence of an electric field. Since gold nanocrystals and DNA are negatively charged in solution, they migrate towards the positive electrode. Agarose gels were used for the experiments, which consist of a fibrous network of agarose chains. As the gel percentage increases, the pore size of a gel decreases, making it more difficult for a particle to find a path through. Likewise, the larger a particle is, the more slowly it will migrate through a gel of a particular percentage.

It is important to have an idea for the forces that affect the mobility of a charged particle. First of all, particle mobility is the velocity divided by the field strength, and free mobility is the mobility of the particle in free solution (i.e., in the absence of a gel). Since charged particles migrate in gels with constant velocity, the net force per particle is zero. Two forces balance in electrophoresis: the electric force and the frictional force. In order to calculate the electric force and the frictional force on a particle, and thus predict its mobility, the ionic double layer 
that surrounds the charged particle must be considered. As was mentioned earlier, the DNA in solution can be thought of as occupying a sphere with a characteristic effective radius (Fig. 4.1). Since the particles are negatively charged, positive ions from the solution gather around the particles to balance the charge. The double layer thickness, labeled as one over kappa, of this charged layer is characteristic of the ionic strength of the solution:

$$
\begin{gathered}
1 / \square=\left(\left[R T / F^{2} I\right)^{1 / 2}\right. \\
I=1 / 2 \square z_{i}^{2} m_{i}
\end{gathered}
$$

where $1 / \square$ is the double layer thickness, $\square$ is the electric permittivity of the solvent, $\mathrm{R}$ is the gas constant, $\mathrm{T}$ is the absolute temperature, $\mathrm{F}$ is the Faraday constant, I is the ionic strength, $z_{i}$ is the charge of the ith ion, and $m_{i}$ is the concentration of the ith ion. This double layer plays several roles in determining the mobility of the particles. First, it screens the particles from the field, so the field that acts on the particles is less than the applied field. This effect is greater for larger particles. Second, since the double layer has a charge opposite that of the particles, it tends to migrate in the opposite direction, leading to increased friction on the particles. This effect is also greater for larger particles. This leads to the phenomenon that particles with the same charge density have the same free mobility, regardless of size [99].

These effects can be better understood by inspecting gels for a variety of DNA and gold sizes. See Figure 3.2 for a typical image of a $3 \%$ agarose gel of gold nanocrystal/DNA conjugates. The binding of each DNA to a nanocrystal 
decreases the particle mobility in a discrete way, making it possible to isolate highly purified samples of specific conjugates from the gel.

\section{Experimental Methods}

I synthesized the gold nanocrystals for these experiments using the common reduction of tetrachloroauric acid using sodium citrate and tannic acid. A dilute solution $(0.01 \% \mathrm{w} / \mathrm{v})$ of the salt is heated and then reduced with a mixture of tannic acid $(1 \% \mathrm{w} / \mathrm{v})$, sodium citrate $(1 \% \mathrm{w} / \mathrm{v})$ and potassium carbonate $(2.5 \mathrm{mM})$ after which the colloid forms; the colloid is deep red in color. The nanocrystals used to study conjugate mobility ranged in size from 4.5 to 50 nanometers in diameter. Each nanocrystal is stabilized by adsorbed negatively charged citrate ions. The nanocrystals are then coated with negatively charged bis-(psulfonatophenyl)phenylphosphine dihydrate dipotassium salt. A centrifuge and $\mathrm{NaCl}$ precipitation is then used to concentrate the nanocrystals to working concentrations. Typical size distributions were $10 \%$ for the smaller particles, and were larger for the larger particles. Also, while the smaller particles were nearly spherical, the larger particles began to show a more ellipsoidal shape, which ultimately limited the maximum size that could be used. The gold nanocrystal/DNA conjugates were synthesized using the same procedure as described in Chapter III. 
Electrophoresis was conducted in agarose gels using 0.5X TBE buffer. Gel concentration was carefully controlled to allow comparison of different gels. All gels were run at $6.7 \mathrm{~V} / \mathrm{cm}$ for $1 \mathrm{hr}$. As already mentioned, the reddish color of the gold colloid allows band identification of free nanocrystals and conjugates, while DNA bands were visualized by staining the gel with GelStar nucleic acid stain (BioWhittaker Molecular Applications). The center of the band was the reference to measure nanocrystal mobilities.

\section{Results}

\subsection{Gel Electrophoresis Data}

Ferguson plots for free ssDNA, unconjugated gold nanocrystals, and corresponding conjugates have been obtained for gel concentrations between $0.6 \%$ and $7.2 \%$. But since these plots are constructed from data obtained from gels, it is useful to look at the data in its raw form. It is well known that DNA of different lengths has the same free mobility. Figure 4.3 shows data for three different lengths of DNA after migrating in five different gel percentages. Data for 50,80 , and 100 base ssDNA in $0.6 \%, 1.2 \%$, up to $3.6 \%$ gels is shown. There are several features of these data to note: first, note the difference in mobility for the DNA in the $3.6 \%$ gels (top line). Compare the slope of that line to the slope of the line for the $0.6 \%$ gels (bottom line). Note that the slope is greater for the $3.6 \%$ line than the $0.6 \%$ line. The slope of this line goes to zero as the gel 


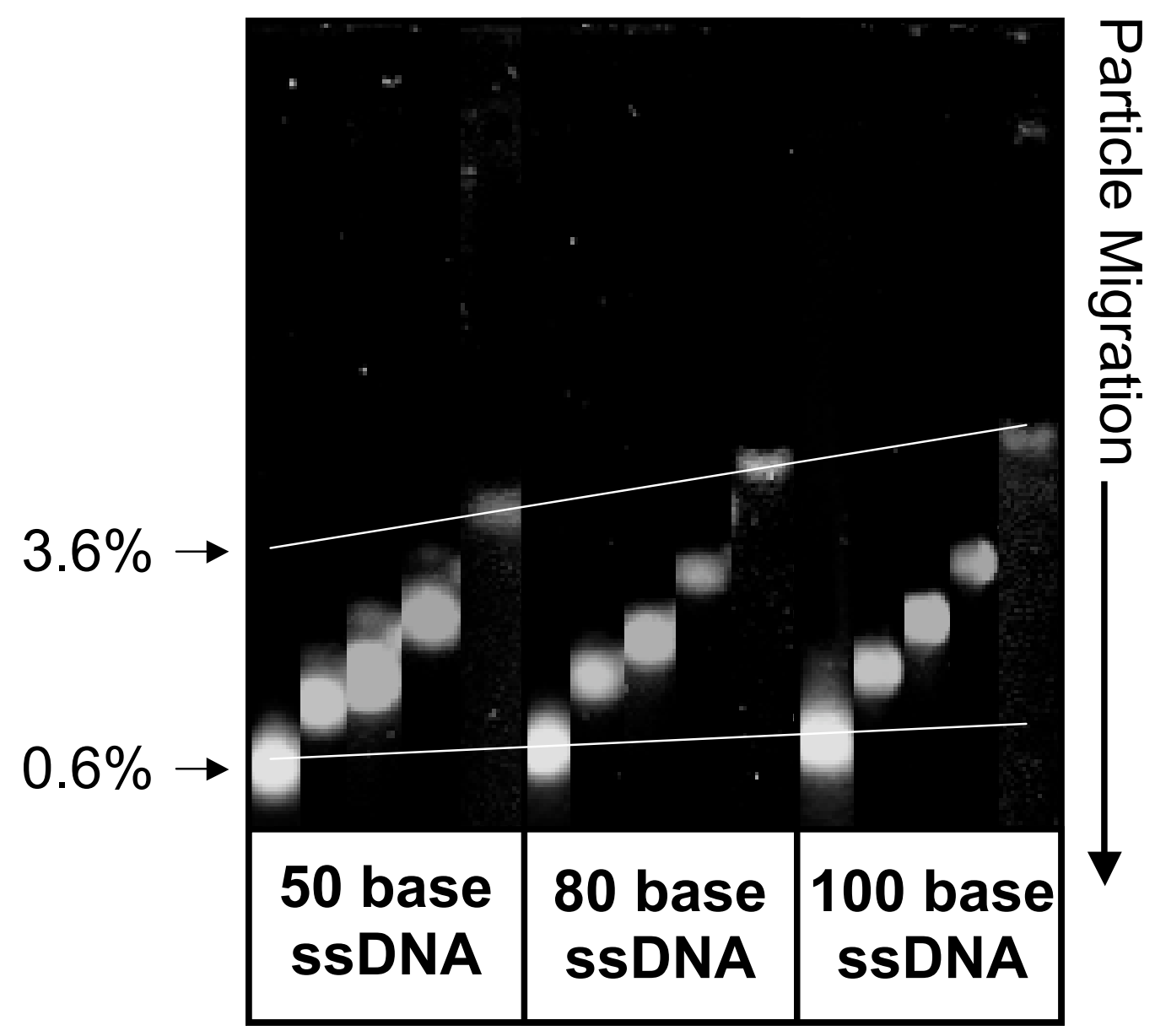

Figure 4.3 Comparison of electrophoretic mobility of 50, 80, and 100 b ssDNA. In each column ( $L$ to $R$ ): $0.6 \%, 1.2 \%, 1.8 \%, 2.4 \%$, and $3.6 \%$ gels are shown. Note that the slope $\mathrm{s}_{0.6 \%}<\mathrm{slope}_{3.6 \%}$ and that the difference in mobility between the $0.6 \%$ and the $3.6 \%$ gel is less for the $50 \mathrm{~b}$ DNA than for the $100 \mathrm{~b}$ DNA. 
percentage continues to decrease-i.e. all the DNA has the same free mobility —as has been shown by many researchers in the past [99]. The second feature of this data deals with DNA mobility in gels: the closer the size of the characteristic sphere of the coiled DNA is to the pore size, the more strongly it is retarded in the gel. As the gel percentage increases, the pore size decreases. Typical pores range from 20 to $150 \mathrm{~nm}$ [99]. This effect can be observed by noting the change in retention between the $0.6 \%$ to the $3.6 \%$ gels in the bands for the different nanocrystal sizes. Since the particle to pore size ratio is always smaller for the 50 base DNA, their mobilities are not affected as much by a change in gel percentage as for 100 base DNA, so the change in retention for the 50 base DNA is smaller than that for the 100 base DNA.

While it has been well known that DNA of different lengths has the same free mobility, mobility of nanocrystals had not been studied, and so it was observed that different sizes of gold nanocrystals also are likely to have the same free mobility. Again, the main factor that causes this effect is the electric double layer that surrounds the particle. In Figure 4.4, data for four different sizes of gold were assembled after migrating in the same gel percentages as for the DNA. Data for 4.5-, 9.3-, 23- and 50-nm gold nanocrystals are shown. Again, note the difference in slopes for the difference in mobilities for the $3.6 \%$ and $0.6 \%$ gels. The slope of this line is approaching zero as the gel percentage continues to decrease. Also, note the change in retention from the $0.6 \%$ to the $3.6 \%$ gels in the mobilities for the nanocrystal samples. Since the nanocrystal to pore size 


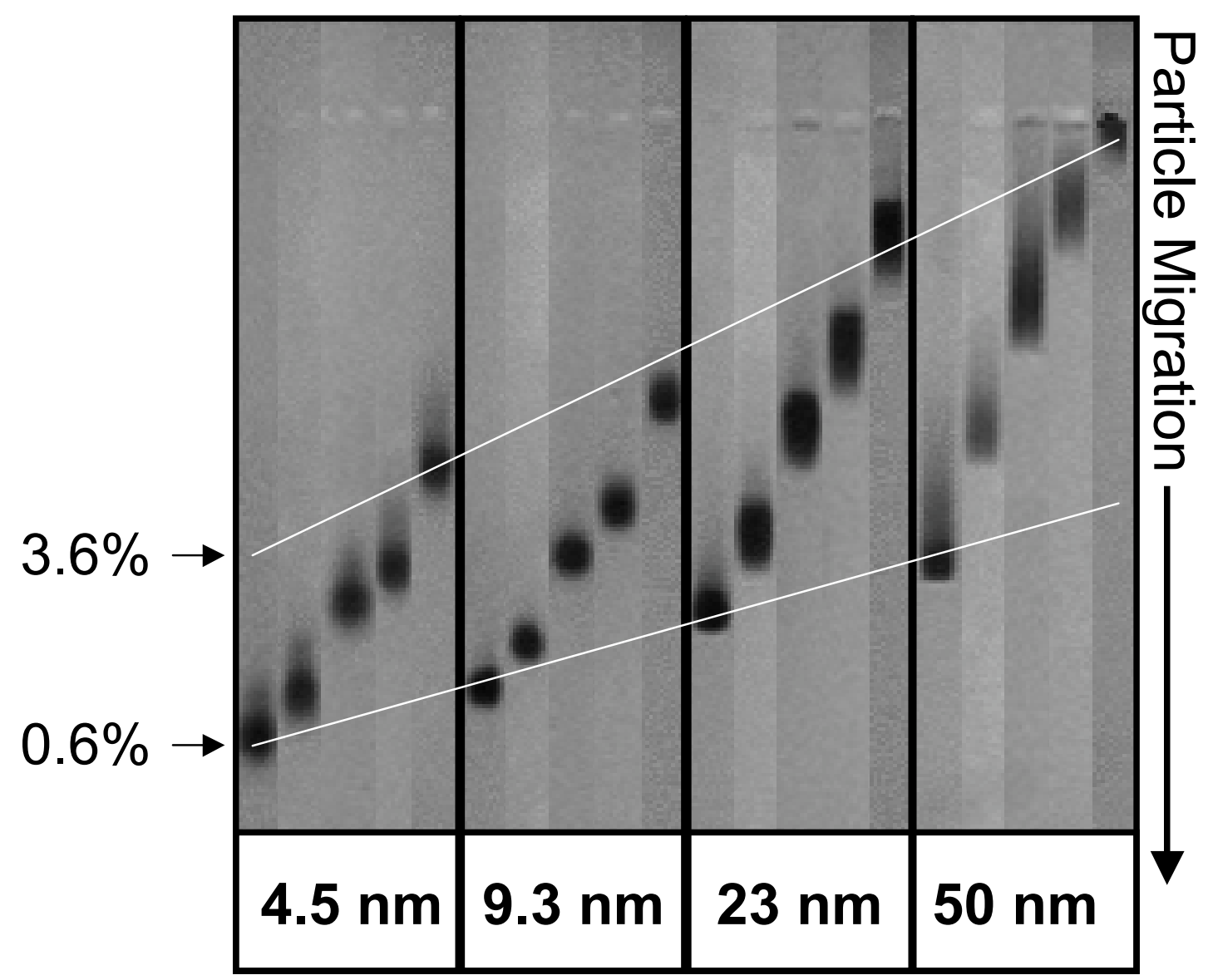

Figure 4.4 Comparison of electrophoretic mobility of 4.5-, 9.3-, 23-, and 50-nm gold nanocrystals. In each column ( $L$ to $R)$ : $0.6 \%, 1.2 \%, 1.8 \%, 2.4 \%$, and $3.6 \%$ gels are shown. Note that the slope $e_{0.6 \%}<$ slope $_{3.6 \%}$ and that the difference in mobility between the $0.6 \%$ and the $3.6 \%$ gel is less for the $4.5-\mathrm{nm}$ gold nanocrystals than for the 50 -nm gold nanocrystals. 
ratio is always smaller for the 4.5-nanometer gold nanocrystals, their mobilities are not affected as much by a change in gel percentage as for $50-\mathrm{nm}$ nanocrystals, so the change in retention for the 4.5-nm nanocrystals is smaller than that for the $50-\mathrm{nm}$ nanocrystals.

\subsection{Ferguson Plot Analysis of Gel Electrophoresis Data}

Ferguson plots, in which the natural logarithm of mobility is plotted against gel percentage [102-103], provide information about the interplay between particle size and charge. For low gel concentrations $(<1 \%)$, the charge of the particle is the determining factor of its mobility, while for higher gel concentrations $(>3 \%)$, the particle size is the dominant factor. A typical plot of this system is given in Figure 4.5. The Ferguson plot originates in the free volume model of gel electrophoresis, in which the mobility of a particle is proportional to the fractional free volume in a gel. This corresponds to the following mobility equation:

$$
\ln (\square)=-\mathrm{k}_{\mathrm{r}} \mathrm{c}+\ln (\square 0)
$$

where $\square$ is the mobility $\left(\mathrm{cm}^{2} \mathrm{~V}^{-1} \mathrm{~s}^{-1}\right), \mathrm{k}_{\mathrm{r}}$ is the retardation coefficient, $\mathrm{c}$ is the gel concentration, and $\square_{0}$ is the free mobility. The fractional free volume decreases exponentially with increasing gel percentage, hence the logarithmic scale [103].

For small particles such as our ssDNA, the free volume model is a good description, and the plot is linear. However, the free volume model does not apply well for hard spheres, such as nanocrystals, conjugates, and proteins; in 


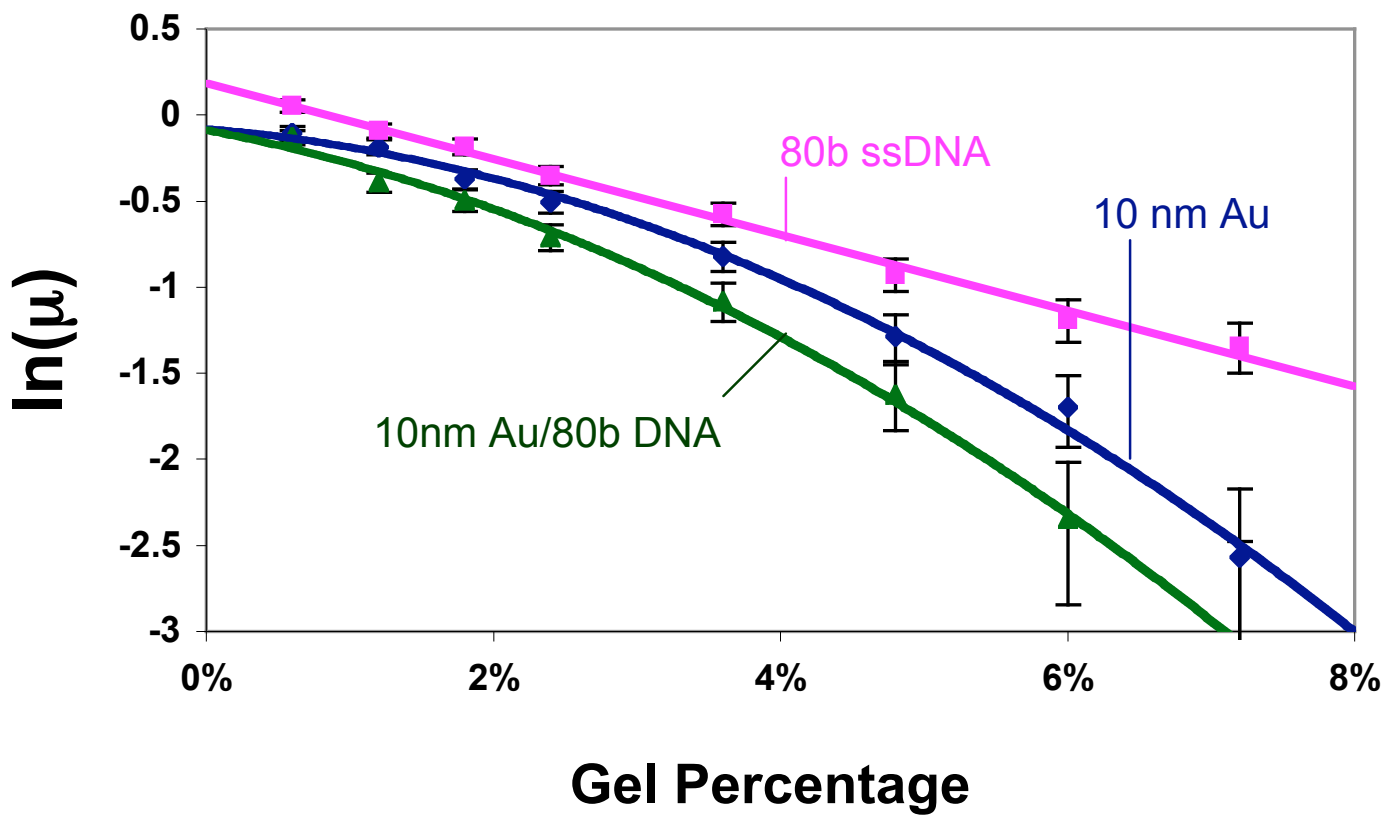

Figure 4.5 Ferguson plots of 80b DNA, 10-nm gold nanocrystals, and 1/10 $n m / 80 b$ DNA 
these cases, the retardation coefficient is a function of gel percentage, and the Ferguson plots are not linear [104]. The main reason is that this simple model does not account for the fact that not all of the free volume is accessible to hard particles that cannot deform [103]. The fact that both free gold nanocrystals and conjugates have similar curves in their Ferguson plots indicates that the electrophoretic mobility of the conjugates are mainly determined by the nanocrystal.

In the next three figures, Ferguson plots for different lengths of ssDNA, different sizes of gold nanocrystals, and gold nanocrystal/DNA conjugates with different numbers of DNA oligonucleotides attached are shown. In Figure 4.6, a Ferguson plot for 50-, 80- and 100-base ssDNA is shown. Note that all of the plots are linear and that the larger DNA has lower mobility. It is also interesting to note that the data approach the same free mobility as the gel percentage is decreased. In Figure 4.7, a Ferguson plot for 4.5-, 9.3-, 23-, and 50-nm gold nanocrystals is shown. Note that the plots are not linear; a non-linear Ferguson plot is what is expected for the case of hard spheres. Also note that larger gold nanocrystals have lower mobilities. Finally, in Figure 4.8, a Ferguson plot for 10nm gold nanocrystals with 100b DNA attached is shown. The controls of free 10$\mathrm{nm}$ gold nanocrystals and free $100 \mathrm{~b}$ ssDNA are shown, in addition to data for gold nanocrystals with one, two, and three DNA strands attached. Note that the curvature observed in the case of gold nanocrystals is retained after attachment 


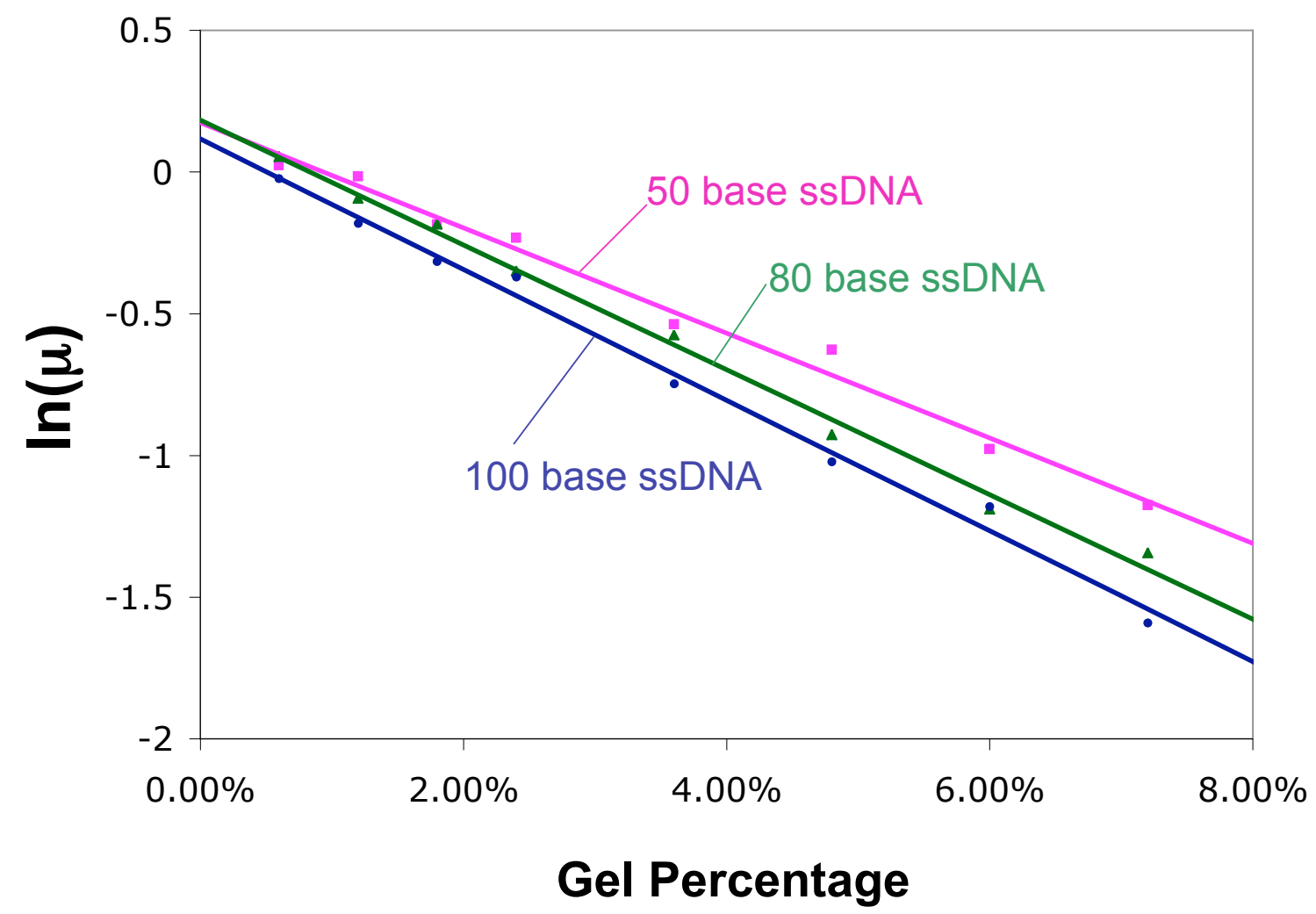

Figure 4.6 Ferguson plots of 50b ssDNA (squares), 80b ssDNA (triangles), and 100b ssDNA (circles). 


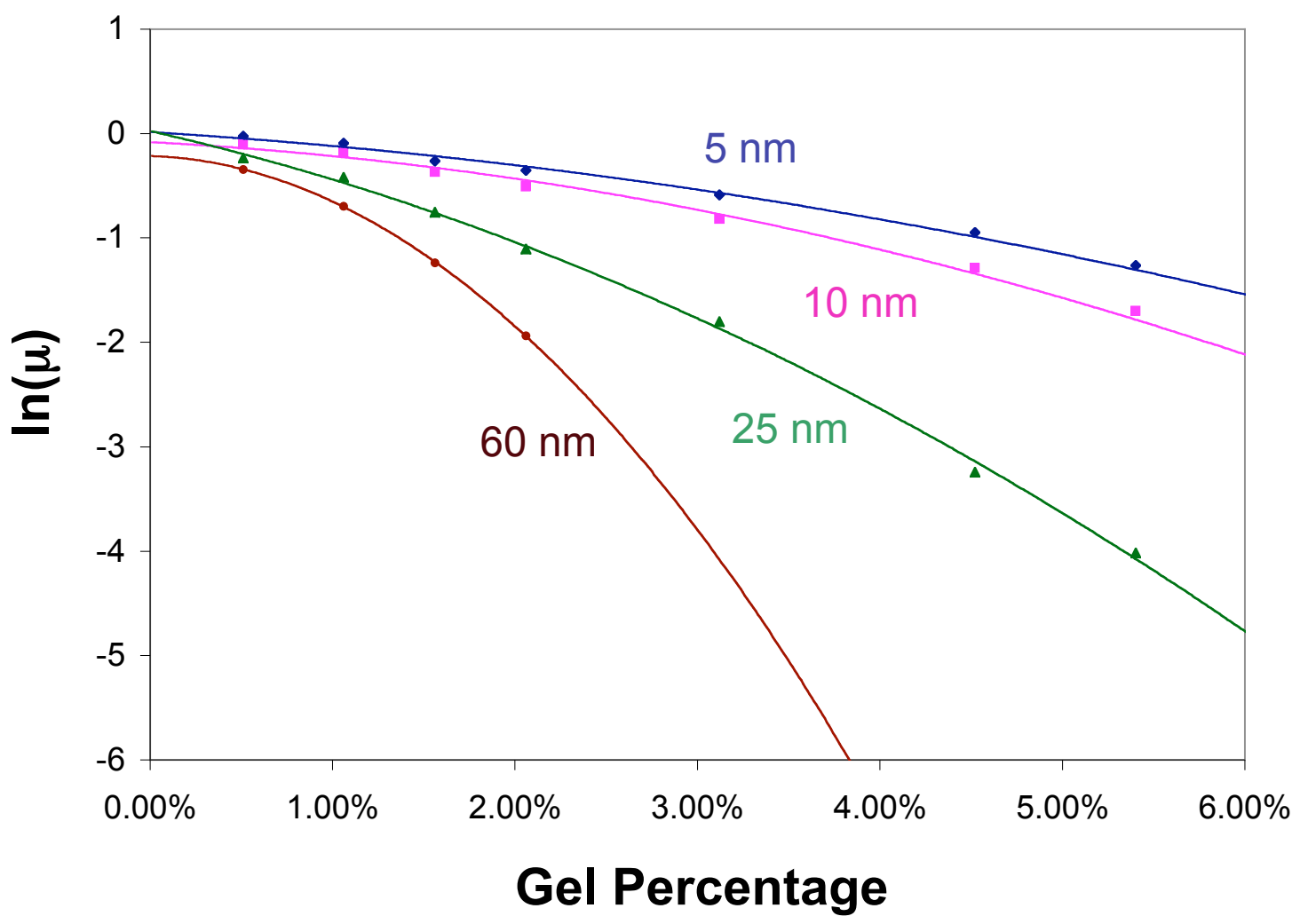

Figure 4.7 Ferguson plots of 4.5- (diamonds), 9.3- (squares), 23- (triangles), and 50-nm (circles) gold nanocrystals. 


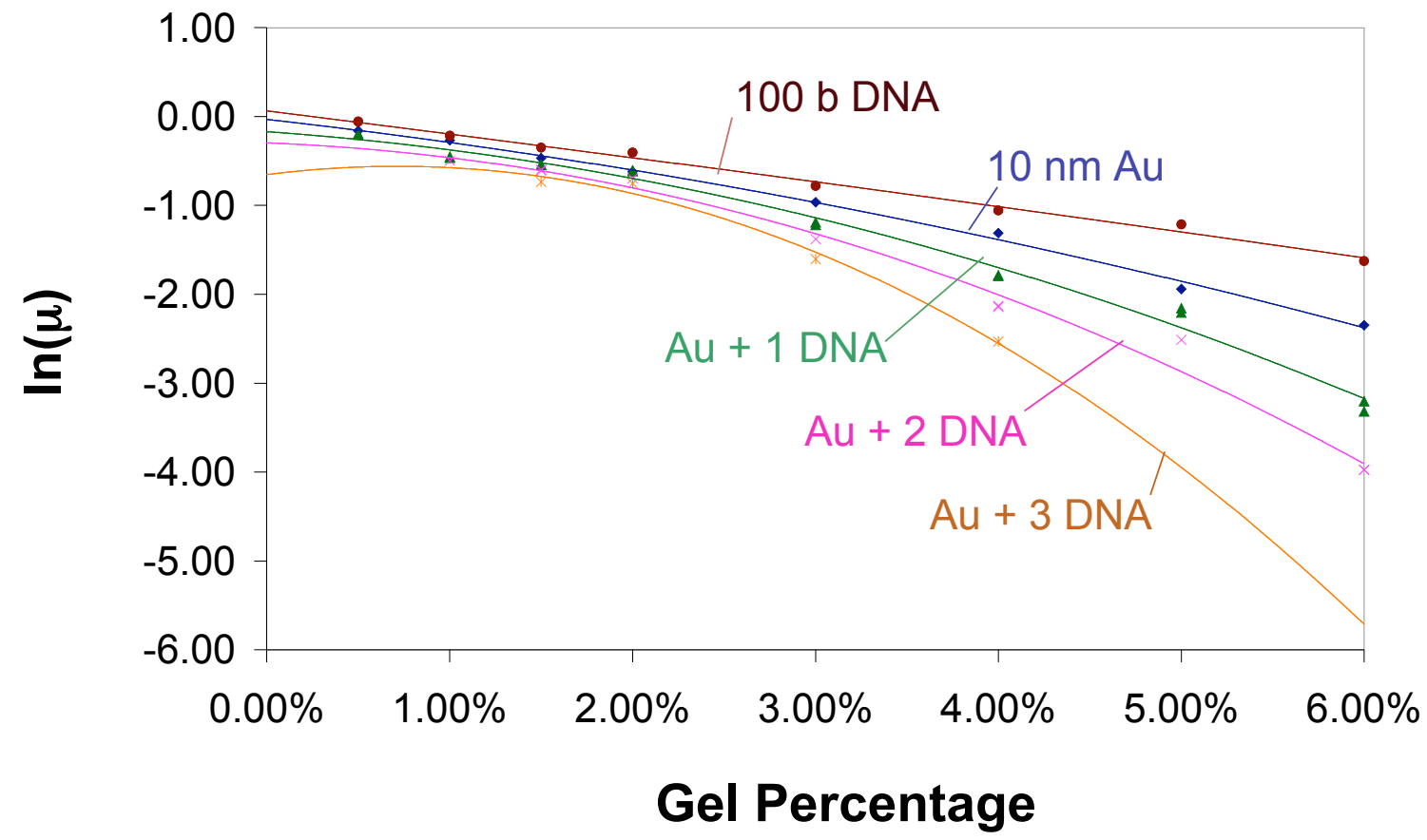

Figure 4.8 Ferguson plots of 100b ssDNA (circles), 10-nm gold nanocrystals (triangles), and 10-nm gold nanocrystals conjugated to one (diamonds), two (“x"s), and three (asterisks) 100b ssDNA strands. 
of DNA, and that the conjugates with more DNA strands migrate more slowly than the nanocrystals attached to fewer strands.

\section{Discussion}

Ferguson plot analysis can also be used to derive the optimum gel concentration for the resolution of the species in a given sample [102]. This is easily applied in biological systems in which the molecules have well-defined size and charge. Unfortunately, this is not the case for nanocrystal samples, for which both size and charge distributions are always present. Increasing the gel percentage and using more stringent conditions does not usually improve resolution due to the spreading of the bands. A similar effect was also found when the gels were run for longer times; the resolution is already determined after $1 \mathrm{~h}$ in this system.

Another interesting characteristic related to electrophoretic mobility of gold nanocrystal/DNA conjugates studied for this work is that they migrate more slowly through gels than DNA or gold nanocrystals alone. This was not predicted beforehand because the attachment of each DNA to the nanocrystal adds both size and charge to the system. The gold nanocrystals are coated by phosphine molecules and are therefore highly negatively charged. In this case, when a DNA is attached to the nanocrystal, the increase in size (volume) seems to be more important than the increase in charge and the overall conjugate has a slower mobility. This is not the case in another system that we have also studied 
$[12,105]$ —silica-coated semiconductor nanocrystals, in which the binding of DNA increases the mobility of the nanocrystals. The main reason for the opposite behavior is the different charge/size ratio of these systems. As opposed to the gold case, the silica-coated nanocrystals are almost neutral particles, and adding DNA gives negative charge to the particles. In the silica case, the particles migrate faster because the increase in charge is more pronounced than the increase in size.

The results obtained from the Ferguson plots and for different nanocrystal/DNA systems suggest that the surface charge on the nanocrystals is a critical parameter. As will be shown in Chapter VI, by changing the surface passivation layer, it is possible to dramatically alter the electrophoretic properties of the gold nanocrystals and conjugates. This is a very interesting property of the system, and it may be exploited, for example, to improve the resolution in the band separation of a desired conjugate. In the future, theoretical models based on charge/size ratio of nanocrystals and conjugates should allow us to predict the band position. The best resolution may also be achieved by optimizing the conditions (nanocrystal charge and gel percentage) for each system.

In order to begin to understand the electrophoretic mechanism of gold nanocrystal/DNA conjugates, it would have been useful to expand the range of DNA lengths studied. All previous work was done in the regime where the DNA is smaller than the average pore size. Developing a model for the 
electrophoresis of conjugates in the current region is difficult for two major reasons: detailed electrophoretic models for DNA as well as rigid spheres in this region are still being developed. In order to overcome this problem, a logical extension of this work is to study the electrophoretic properties of gold nanocrystal/DNA conjugates with longer DNA, for which DNA electrophoretic models are well developed.

There is in fact an existing electrophoretic system that resembles the gold nanocrystal/DNA conjugates, albeit with longer attached DNA. The system and its corresponding model are known as trapping electrophoresis (Figure 4.9). In trapping electrophoresis, separation of single strands of DNA is enhanced by attaching the small spherical protein streptavidin to one end of the DNA. Streptavidin is approximately $8 \mathrm{~nm}$ in diameter. In this model, a percentage of the pores are large enough for DNA to move through, but too small for streptavidin. When the particles are trapped, they rely on Brownian motion, or diffusion, to detrap them. This seems like a reasonable picture for the mechanism of gold nanocrystal/DNA conjugate mechanism. However, gold nanocrystals are different from streptavidin in a number of ways, so it will be interesting to see how gold conjugate mobility differs from conventional trapping electrophoresis. 


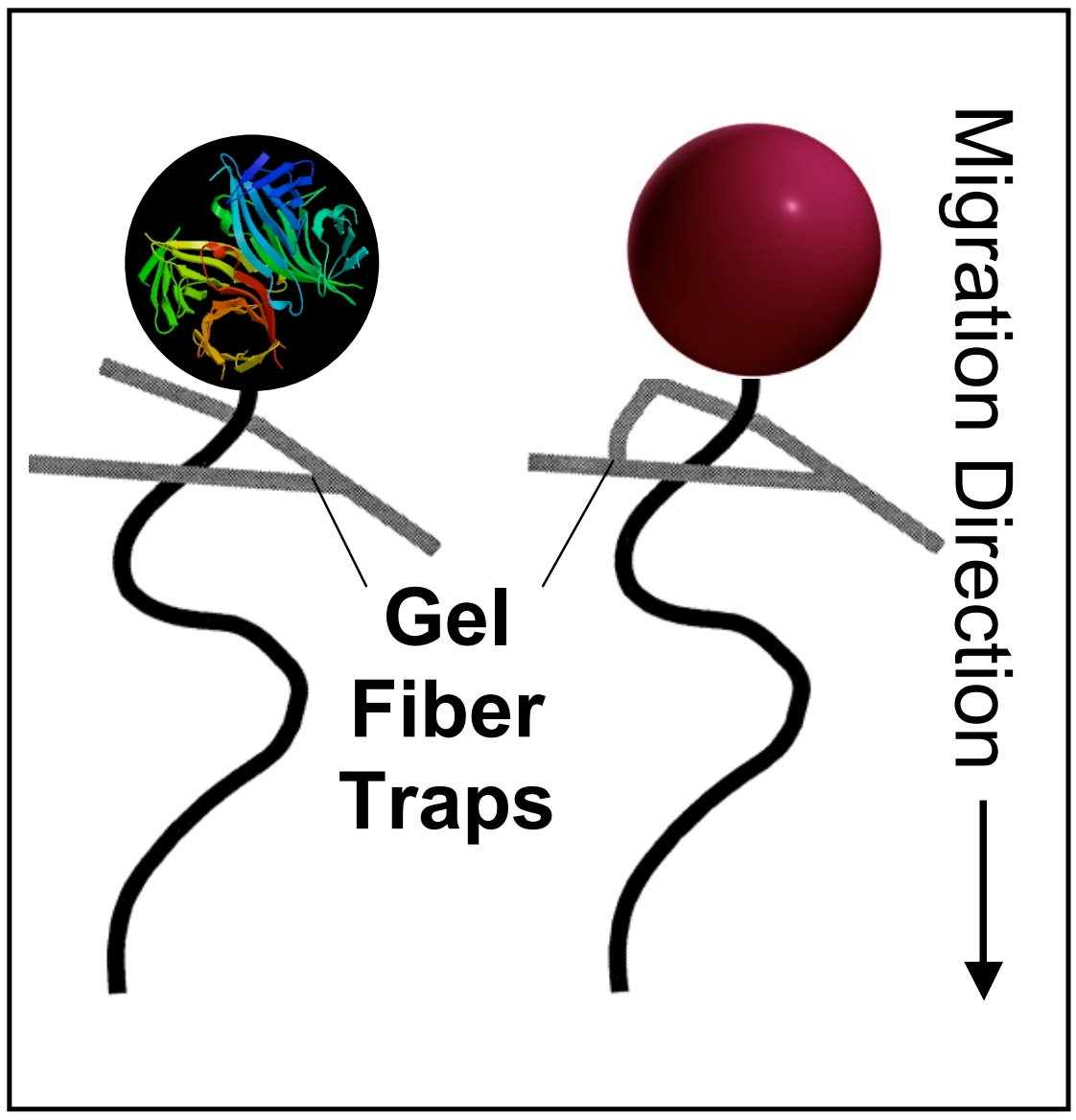

Figure 4.9 Comparison of trapping electrophoresis to electrophoresis of gold nanocrystal/DNA conjugates $[53,106]$. 


\section{Conclusions}

This work helped to elucidate the mechanisms of migration of gold nanocrystals and conjugates through agarose gels and helped to quantify data obtained from gel electrophoresis. The migration of gold nanocrystals is equivalent to the migration of other hard spheres: proteins, in particular. The data collected also allow prediction of mobility for a variety of sizes of gold nanocrystals coated with bis-(p-sulfonatophenyl)phenyl phosphine as well as a variety of gold nanocrystal/DNA conjugates. 


\section{Chapter V}

\section{Correlation Analysis of Data from Transmission Electron Microscopy}

Reproduced in part from Zanchet, D., Micheel, C.M., Parak, W.J., Gerion, D., Williams, S.C., and Alivisatos, A.P. J. Phys. Chem. B 106, 11758-11763, Copyright 2002 American Chemical Society.

\section{Introduction}

Arguably, imaging techniques are the most important methods of characterizing nanostructures. These methods range from simple photography, used for imaging agarose and polyacrylamide gels, to transmission electron microscopy, used for visualization of most nanostructures. While useful qualitative analysis of such images is often straightforward, extending the analysis to yield quantitative information is often challenging.

Over the past several years, the ability to fabricate and characterize nanostructures and the ability to apply the unique characteristics of nanocrystals to biological problems—such as tunable fluorescence wavelength, long fluorescence lifetime, and small size—has improved dramatically. Gel electrophoresis has been used to purify and characterize nanocrystal/biomolecule conjugates and nanostructures. Optical microscopy has been used in DNA arrays, single nucleotide polymorphism detection, and cell motility assays. Atomic force microscopy (AFM) is used to study nanostructures 
constructed from gold nanocrystals, proteins, and DNA. Finally, transmission electron microscopy (TEM) is an indispensable tool for the verification of a wide variety of nanostructures.

The development of the synthesis of gold nanocrystal/DNA nanostructures has taken place over the last ten years. During that time, our ability to characterize the structures using TEM has increased substantially. In the initial work, isolated structures could be seen scattered over a grid, mixed in with various impurities. Later on, it was possible to see fields of structures in single images. As a result of my work presented in this chapter, more quantitative analysis is now possible, and more complex structures will be more easily characterized. My analysis techniques include pair distribution functions, location of higher order structures, and a Monte Carlo simulation of dimer conformations. While some of this data was published in reference 12 , I was the primary researcher working on this project.

\section{Methods}

Gold nanocrystals were purchased from Ted Pella (Redding, CA). Nanocrystals were prepared, and conjugates, dimers, and trimers were synthesized according to the techniques already described. TEM studies were performed using an FEI Tecnai 12 TEM, $100 \mathrm{kV}$, at the Electron Microscope Laboratory, UC Berkeley. Formvar-coated grids were first treated with bacitracin, $500 \mathrm{~g} / \mathrm{mL}$ (Sigma), for 30 
$\mathrm{s}$, and then gold nanocrystal molecule samples (100-500 nM) were dropped and wicked off after $30 \mathrm{~s}$. For pair distribution analysis, grids were also washed with water for $30 \mathrm{~s}$ both after bacitracin coating and after gold sample coating. Digital TEM images were recorded on a low-resolution digital camera (Gatan model 780 dual view camera, $1300 \times 1300$ pixels); approximately 50-100 images were recorded per grid per sample. Magnification and nanocrystal density on the grid (changed via sample concentration in grid preparation) were optimized to provide rapid and accurate analysis.

I developed a computer program to analyze the TEM data. The images were first analyzed using Image Pro Plus graphics analysis software (Media Cybernetics, Inc.). Nanocrystals were counted by the program, and lists of nanocrystal locations and diameter were recorded. These lists were then used as input for LabVIEW (National Instruments) programs I wrote to calculate pair distribution functions from the lists of nanocrystal locations or to determine presence of higher order structures. Because the LabVIEW programs are difficult to read on paper, the programs have not been included in this dissertation. A description of the general steps included in the programs follows, however.

For dimers and heterodimers of nanocrystals, the list of locations and diameters compiled by Image Pro was first sorted according to nanocrystal diameter. For homodimers, all nanocrystals within an appropriate size range were included. For heterodimers, two lists were created: one for each size nanocrystal assumed 
to be included in a dimer. Then, the distances between all nanocrystals for homodimers and between particles in the two lists for the heterodimers in the images were calculated and recorded. Then, distances from this list were sorted into 1-nm bins; the number of pairs in each bin was normalized according to the area of the bin, since bins farther from the nanocrystal have larger area. Pair distribution functions were created for unconjugated 5- and 10-nm nanocrystals, 5/5 dimers, and 10/10 dimers connected with 50b, 80b, and 100b DNA.

The program used to analyze trimers and tetramers worked in a somewhat different manner. Since the pair distribution functions find only correlations between sets of two nanocrystals, I needed another approach. Seven main steps were followed. First, the nanocrystals are again sorted by diameter. Second, a square that has an edge of two times the maximum calculated diameter of the structure being analyzed is drawn, and all nanocrystals within that box are kept for the next step in the program. Third, distances between the initial nanocrystal and the others are calculated, and any nanocrystals that are further than one diameter away are discarded. Fourth, all distances between remaining nanocrystals are checked to determine that all are within one diameter of one another. Fifth, the number of nanocrystals in this structure are counted. Sixth, if it is the same as the number of nanocrystals in the expected structure, it is counted as a structure. Finally, the next step is to verify that none of the nanocrystals have appeared in any other structure, and if they have not, then the 
structure is recorded. It is easy to see why good controls are necessary for this type of analysis. These include free nanocrystals and analysis of sub-structures.

One newer program is able to determine the presence of higher order structures including semiconductor as well as gold nanocrystals. In this case, the semiconductor nanocrystals are significantly smaller than the gold nanocrystals, so multiple lists are created and compared, in a way similar to the case of heterodimers of nanocrystals explained above.

I wrote the Monte Carlo simulation of the conformations of gold nanocrystal dimers connected with DNA in the $\mathrm{C}$ programming language. While these programs are much easier to understand and print in a dissertation than the LabVIEW programs, the code and its backup were stolen along with my computer in October of 2003 , so any further work on this topic will require the code to be rewritten. And, unfortunately, it cannot be included here. I will provide a description of the program, however. For this code, the DNA was modeled as a cylinder with a 2-nm diameter; charges were spaced such that the surface charge density was the same as for DNA in 0.5X TBE buffer. The DNA was linked to the nanocrystals at each end by a fully flexible $\mathrm{C}_{6}$-thiol linker attached to the ends of the cylinder and to the nanocrystal. The nanocrystals were spheres decorated with charges so that the surface charge density was one negative charge per square nanometer, a number determined by calculating the height, cone angle, and thus the area required for each bis-(p- 
sulfonatophenyl)phenyl phosphine salt ligand, and then calculating the number of charges that can fit on a nanocrystal. Sterics were defined such that the $\mathrm{C}_{6}$-thiol linker was restricted to appropriate bond angles, although dihedral interactions between carbon atoms were not included. The primary steric interaction was assumed to be between the gold nanocrystals and the DNA strand. Monte Carlo simulations were run at room temperature and were conducted for dimers with no interactions, dimers with only electrostatic interactions, dimers with only steric interactions, and for dimers with both electrostatic and steric interactions. The initial conformation of the dimer was random, and simulations were allowed to run for several hundred to several thousand steps, after which the distance between the gold nanocrystals was calculated. The simulation was run several thousand times per sample, and all of the results were compiled into a pair distribution function, like those compiled for dimer distances calculated from TEM data.

\section{Results}

Pair distribution functions for homodimers and heterodimers are presented, along with structure analysis for trimers, tetramers, and semiconductor nanocrystals surrounded by different numbers of gold nanocrystals. For TEM images of these materials, please see Chapter II or the appropriate reference $[8,20]$. 
The results for $5 / 5$ and $10 / 10$ dimers as a function of DNA length $(50 \mathrm{~b}, 80 \mathrm{~b}$, and 100b) are presented. Figure 5.1 shows the pair distribution functions for unconjugated 5- and 10-nm nanocrystals (top row) and the dimers (bottom three rows), in which several common features can be seen in all plots. First, for all of the cases, there is a peak at the diameter of the nanocrystals. This corresponds to the distance between the centers of aggregated nanocrystals; all grids show a small degree of aggregation due to the intrinsic difficulty of uniform spreading of the nanocrystals on a TEM grid. Second, a constant background shows up for larger distances due to the random placement of nanocrystals on the grid. However, on the dimer plots (Figure 5.1—bottom three rows), an additional feature appears that shifts to larger distances as a function of DNA length, corresponding to the interparticle distancesof the dimers. Consider the case of 5/5 100b DNA dimers (Figure 5.1—bottom left): the expected interparticle distance can vary from $41 \mathrm{~nm}$ down to $27 \mathrm{~nm}$ because of the floppiness of the 5'end thiol linkers (the 100b DNA length is approximately $34 \mathrm{~nm}$, the linkers are roughly $1 \mathrm{~nm}$ each). Note that the persistence length for hybridized DNA is about $50 \mathrm{~nm}$, so 100b, $34 \mathrm{~nm}$ in length, is a nearly rigid molecule. In Figure 5.1, all dimer cases show a peak in the range of possible interparticle distances.

Figure 5.2 shows $5 \mathrm{~nm} / 10 \mathrm{~nm}$ gold nanocrystal heterodimers in the right column and homodimers analysed as if they were heterodimers in the left column. It can easily be seen that heterodimers are not detected when none are present. 


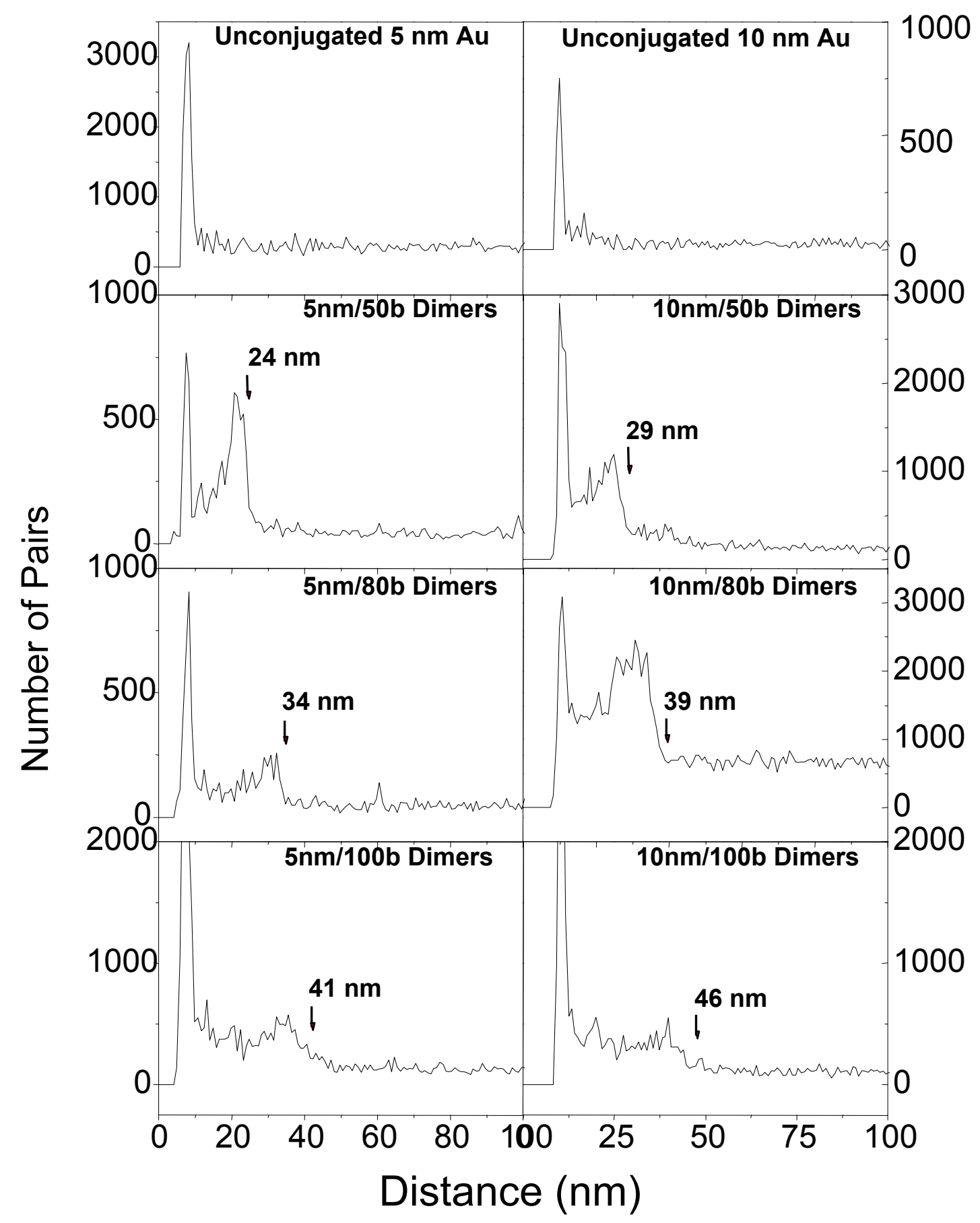

Figure 5.1 Pair distribution functions. The second peak corresponds to the interparticle distance in the corresponding dimers. The arrows for dimer distributions highlight the calculated maximum length of the dimers. 


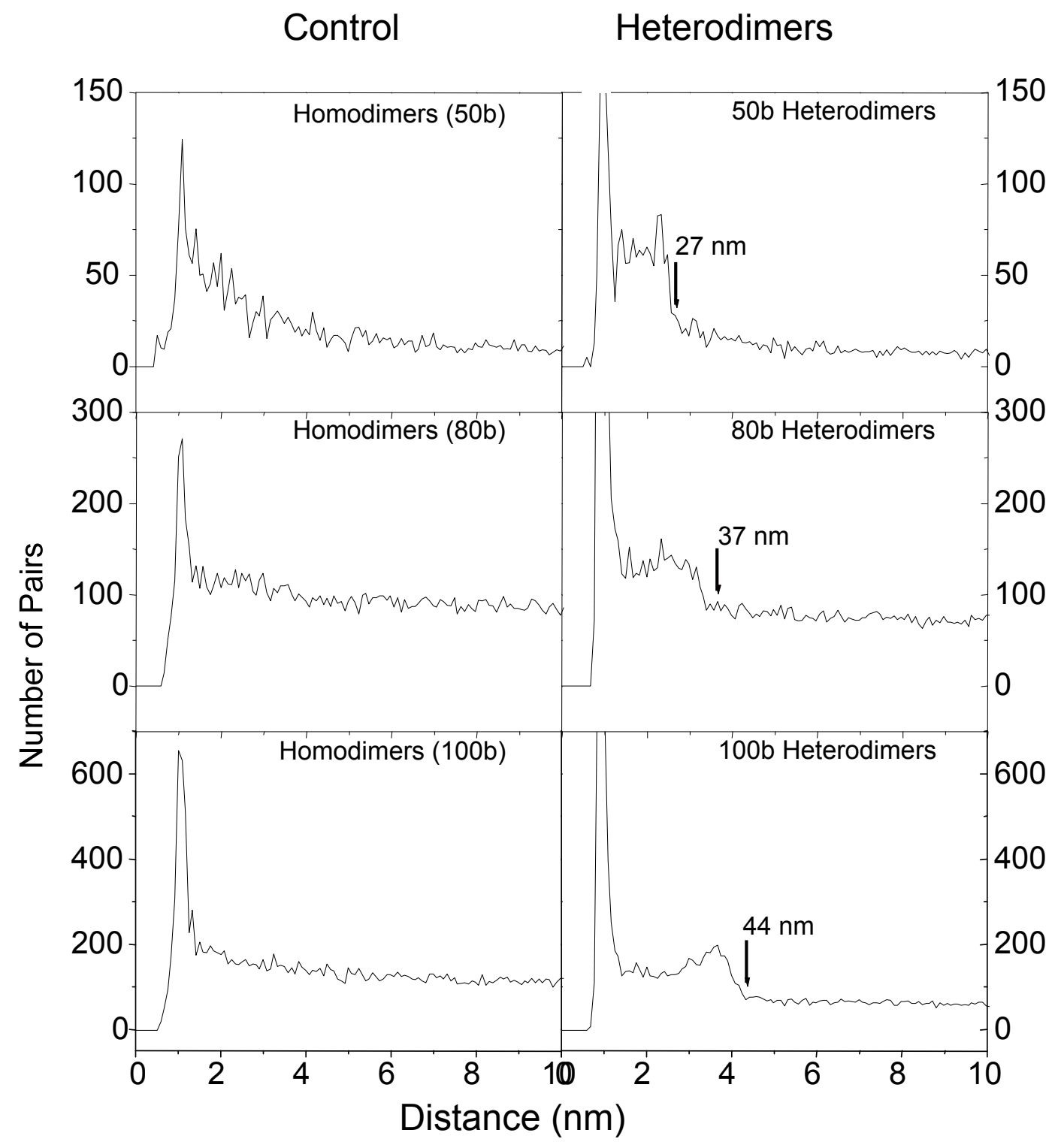

Figure 5.2 Pair distribution functions of heterodimers. The left-hand column shows 5-nm homodimers analyzed with the heterodimer program. The righthand column shows 5-10 heterodimers. The second peak (present only in the right-hand column) corresponds to the interparticle distance in the corresponding dimers. The arrows for dimer distributions highlight the calculated maximum length of the dimers. 
A few additional controls helped to further understand the results of the pair distribution function data. First, noise in the functions is inversely proportional to the square root of the number of pairs, as expected. So, in order to decrease the noise by half, it would be necessary to square the number of pairs analyzed.

Second, pair distribution functions of purified dimers mixed with varying percentages of free gold nanocrystals were constructed. These distribution functions showed that while an increased fraction of dimers in a sample resulted in a higher fraction of dimers represented in the pair distribution function, it was difficult to precisely determine yields from pair distribution functions.

I also analyzed trimers with the structure analysis programs. Images such as those in Figure 5.3 were analyzed to determine percentages of trimers and other structures present. The trimer sample was compared to a control, where images of free 5-nm gold nanocrystals were analyzed using the trimer program. As can be seen in the figure, the trimer sample showed a much higher percentage of trimers than the sample of free particles. Nonetheless, care must be taken when preparing samples for the TEM. If the samples are too concentrated and structures are too close together on the grid, they will not be accurately counted, since the program is unable to tell the difference between structures that are too close together and structures that are not the intended structure; the program will undercount the structures in this case. This is part of the intent of this program, since a researcher's bias can sometimes see structures that are not objectively 

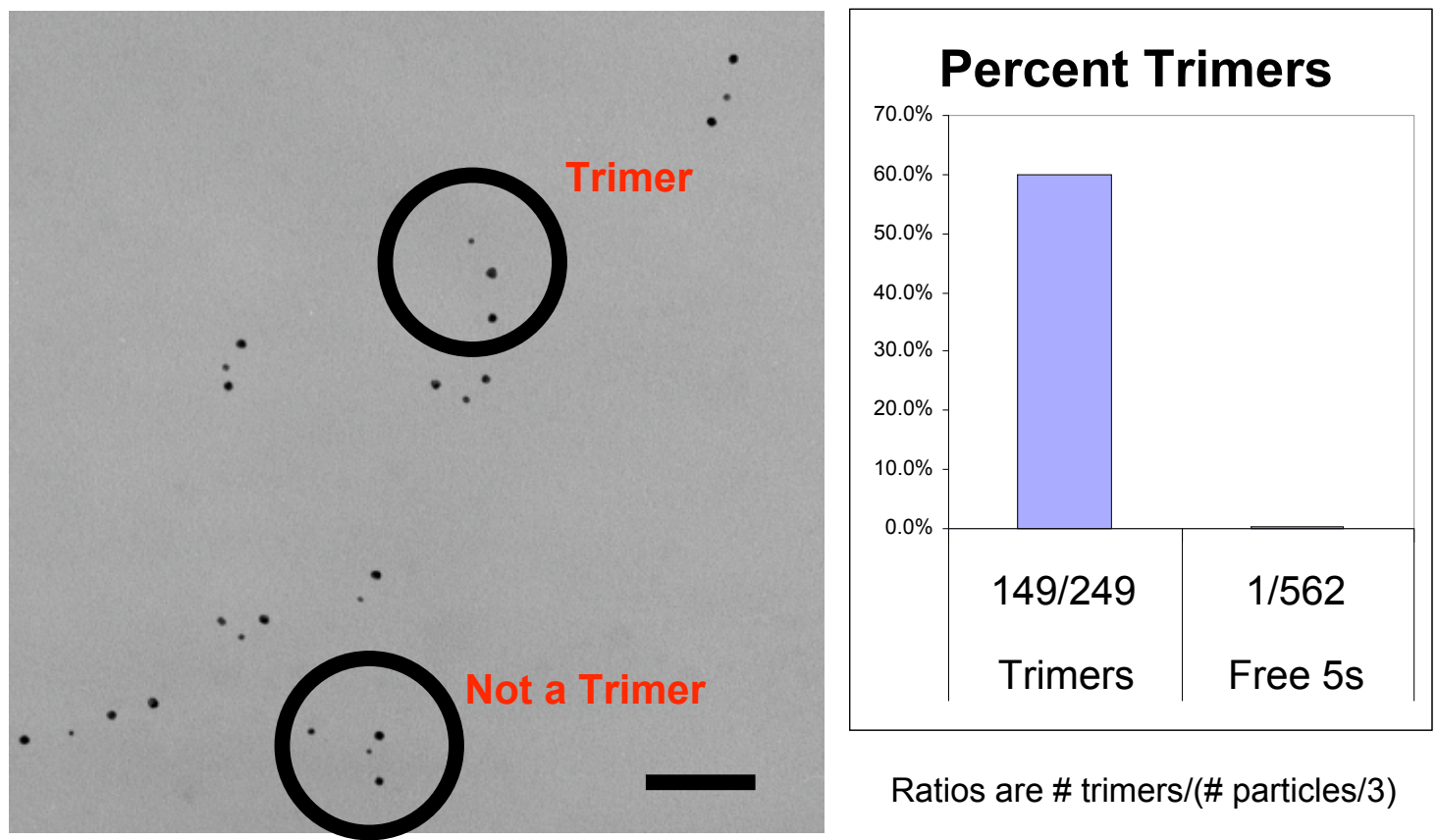

Ratios are \# trimers/(\# particles/3)

Figure 5.3 On the left is a TEM image of a trimer sample. The top circle indicates a trimer that would be counted by the program. The bottom circle shows a probable trimer that would not be counted due to the proximity of a free particle. The right-hand side shows the results of the analysis of a trimer sample compared to a control sample of free nanocrystals. Scale bar is $50 \mathrm{~nm}$. 
there, even though it may seem very convincing. There are no circumstances under which the program would systematically overcount the structures.

Structure analysis has also been conducted for trimers and tetramers of gold nanocrystals connected with branched DNA. For more information on these structures, see reference 8 . As can be seen in Figure 5.4, data obtained for samples of symmetric trimers, asymmetric trimers and symmetric tetramers were obtained. These data helped to identify the bands in the gels, as well as strategies for improving yields. The final results can be seen in the published work [8].

Figure 5.5 shows both pair distribution function analysis and structure determination analysis for gold nanocrystals linked to semiconductor nanocrystals with DNA. The first set is for a semiconductor nanocrystal linked to two gold nanocrystals with 50b DNA, the second is for a semiconductor nanocrystal linked to two gold nanocrystals with 100b DNA, and the third is for a semiconductor nanocrystal linked to three gold nanocrystals with 100b DNA. As you can see, distances between semiconductor and gold nanocrystals are greater in the cases of 100b DNA linkage, and semiconductor nanocrystals with two gold nanocrystals or semiconductor nanocrystals with three gold nanocrystals are the most prevalent species in their respective samples. 

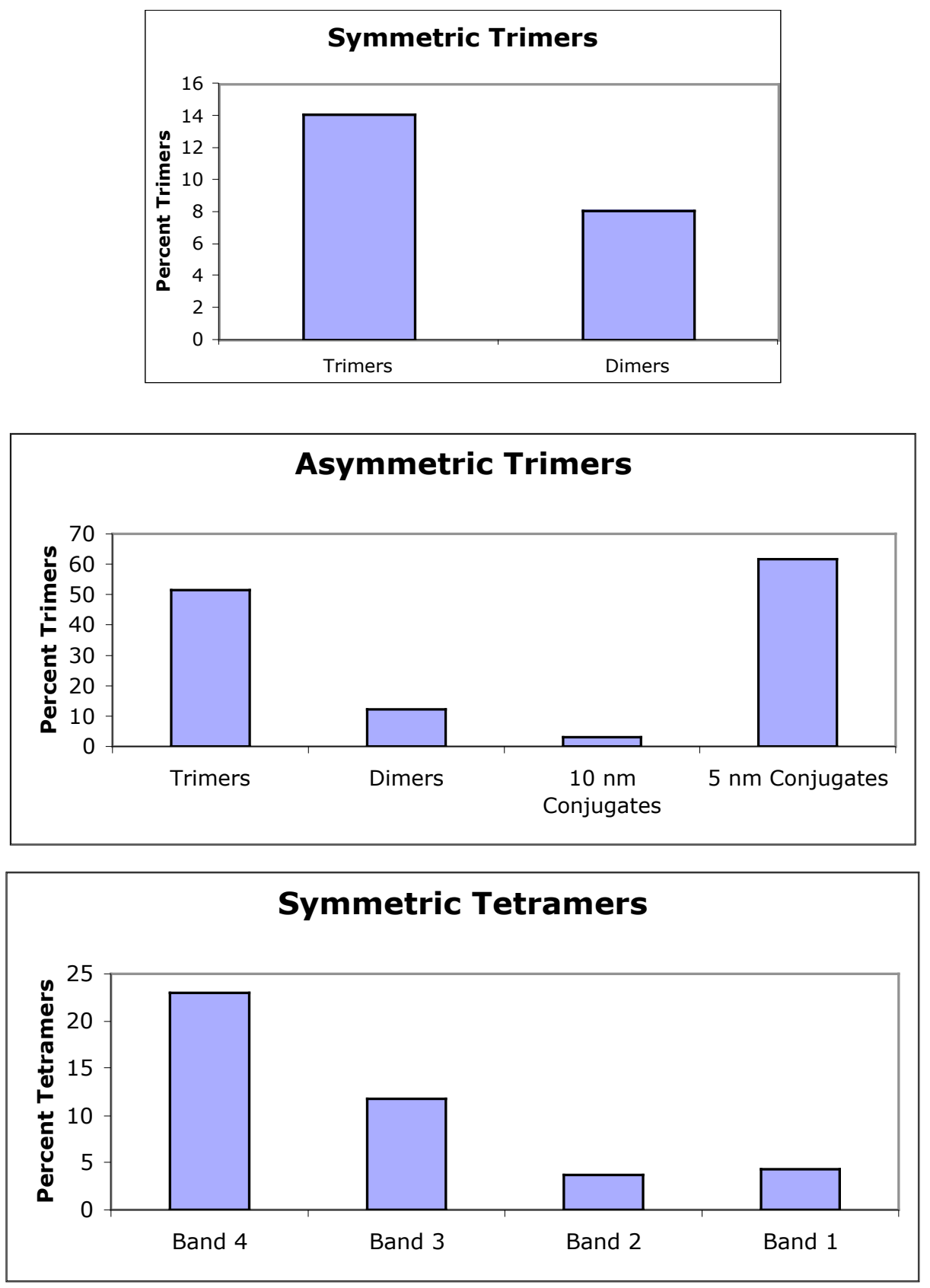

Figure 5.4 The results of analysis of three types of structures constructed from branched DNA (see reference 8). In these cases, percentage of particles in trimers (top two charts) and tetramers (bottom chart) were analyzed for several bands purified from agarose gels. 
(a)

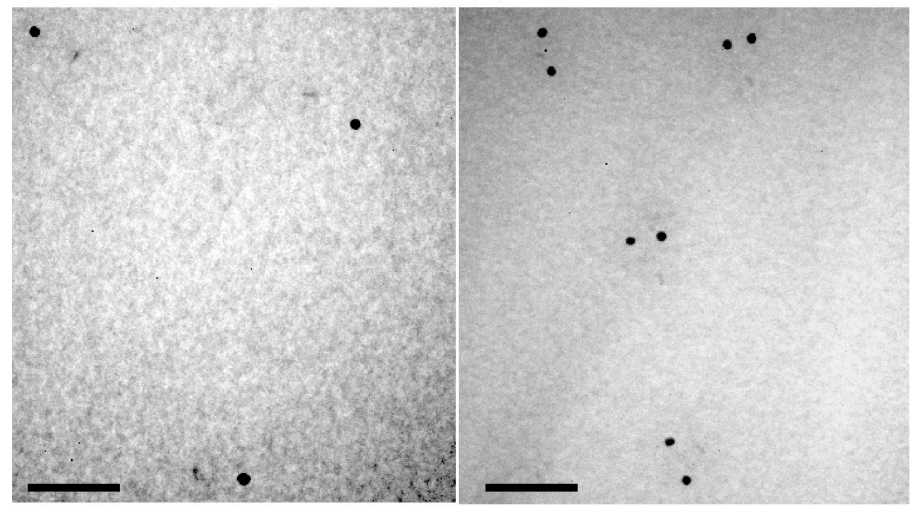

(c)

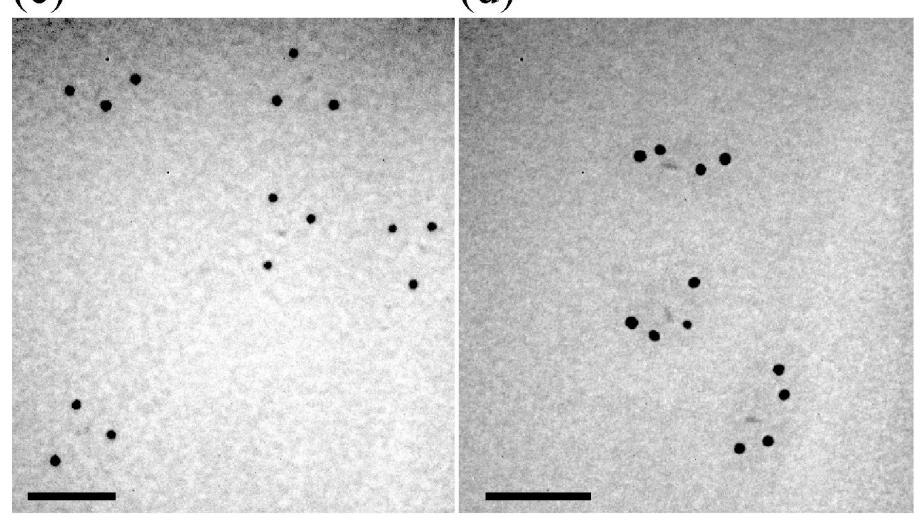

(e)

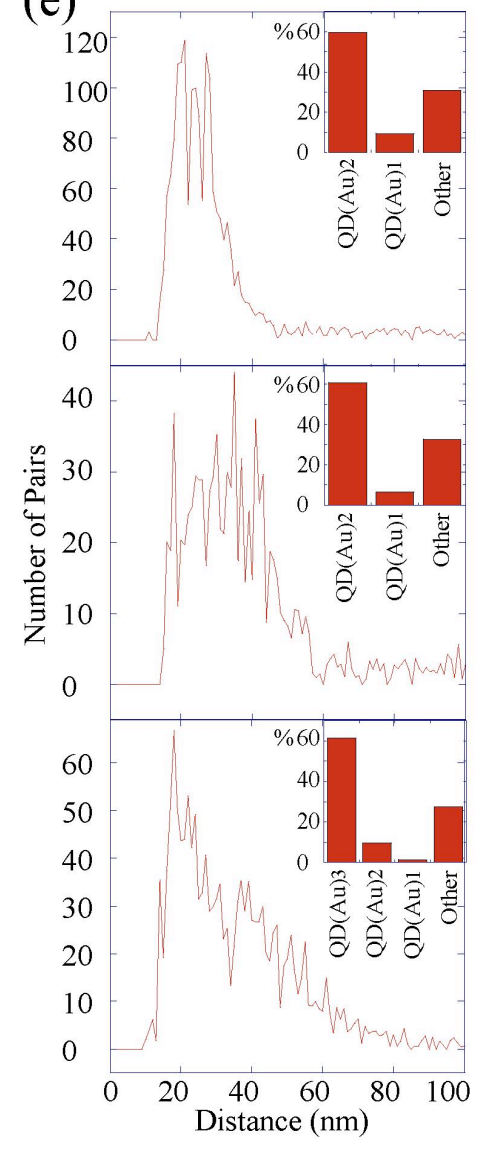

Figure 5.5 TEM images of discrete nanostructures of gold nanocrystals (Au) connected to semiconductor nanocrystals (QD). (a) $\mathrm{QD}(\mathrm{Au})_{1}$. (b) $\mathrm{QD}(\mathrm{Au})_{2}$. (c) $\mathrm{QD}(\mathrm{Au})_{3}$. (d) $\mathrm{QD}(\mathrm{Au})_{4}$. The scale bar is $100 \mathrm{~nm}$. (e) Structure populations and pair distribution functions of $\mathrm{QD}(\mathrm{Au})_{2}$ connecting with 50mer DNA (top), 100mer DNA connected $\mathrm{QD}(\mathrm{Au})_{2}$ (middle), and $\mathrm{QD}(\mathrm{Au})_{3}$ structures (bottom). 
A Monte Carlo simulation was developed as an attempt to understand the position of the peak in the pair distribution functions of dimers. In all cases, the

dimers showed conformations slightly less than completely stretched. Figure 5.6 shows the results of the simulations. As can be seen from the first graph, sterics, as tested in these simulations, had very little overall effect on the conformation of the dimers, while electrostatics played a substantial role. As can be seen from the second graph, the simulation was a good approximation for the experimental data, and so it could be concluded that electrostatics are the primary determinant for the conformation of the dimers.

\section{Conclusions}

Programs were developed to quantify data from transmission electron microscope data. This allowed analysis of more complex nanostructures than was previously possible, due to reproducible and unbiased methods. These programs are able to interpret images of dimers, trimers, and higher-order structures. They are also able to interpret images of structures containing multiple nanocrystal sizes and materials.

The development of new isolation and characterization tools for nanocrystal/biomolecule structures must be continued. In particular, it will not be possible to isolate or probe large structures by gel electrophoresis because of the finite gel pore size. Computer programs to analyze TEM images, such as the 


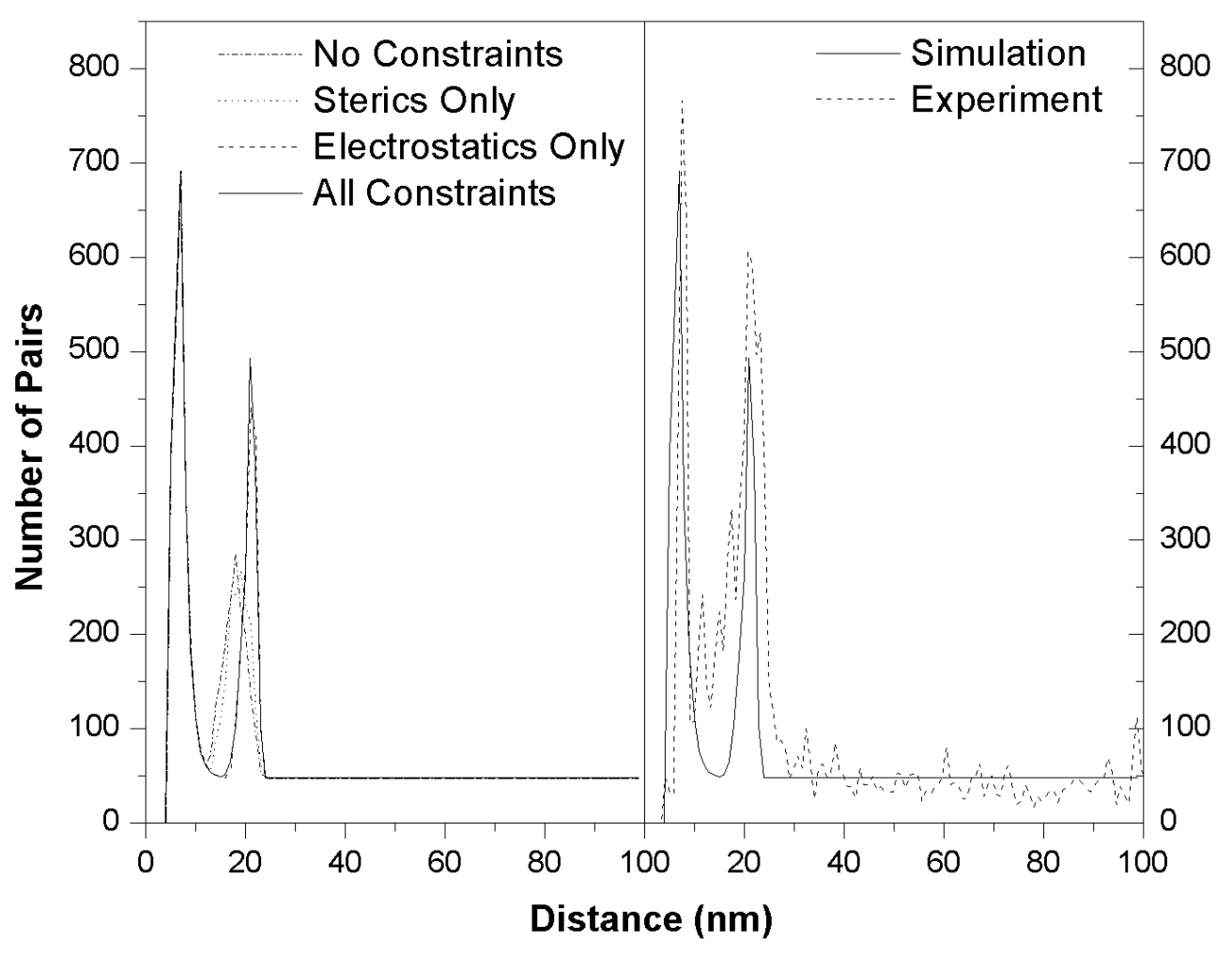

Figure 5.6 Monte Carlo simulation of pair distribution function of dimers. 
ones presented here, are good ways to probe such structures. Techniques such as ultracentrifugation, liquid chromatography, capillary electrophoresis, field flow fractionation and dynamic light scattering (DLS) may also give important information. A further possibility for flat structures is to image with atomic force microscopy, targeting not only the nanocrystals but also the DNA linker. 


\section{Chapter VI}

\section{Progress Towards Nano-Amplification}

\section{Introduction}

The use of enzymes opens up a wide variety of new methods for construction and application of nanomaterials. While enzymes have been used to manipulate gold coated with DNA [28, 107], as well as monofunctionalized 1.4-nm gold nanocrystals [51], enzymes have not been used to manipulate discrete gold nanocrystal/DNA conjugates, such as those used to make the dimers and trimers of nanocrystals. My goal in this project was to synthesize dimers of gold nanocrystals linked with a single strand of DNA and then use those dimers as templates for the creation of additional single-strand dimers. Using gold nanocrystal/DNA conjugates as the building blocks and DNA ligase as the linking enzyme, it might then be possible to amplify the structures using a ligase chain reaction (LCR), analogous to the polymerase chain reaction (PCR) used to amplify DNA (Figure 6.1). If such a protocol were developed, it could be used to exponentially amplify the concentration of a nanostructure in solution.

A further goal would be the amplification of more complex structures, specifically a tetramer of gold nanocrystals linked with single-strand DNA (Figure 6.2). In 


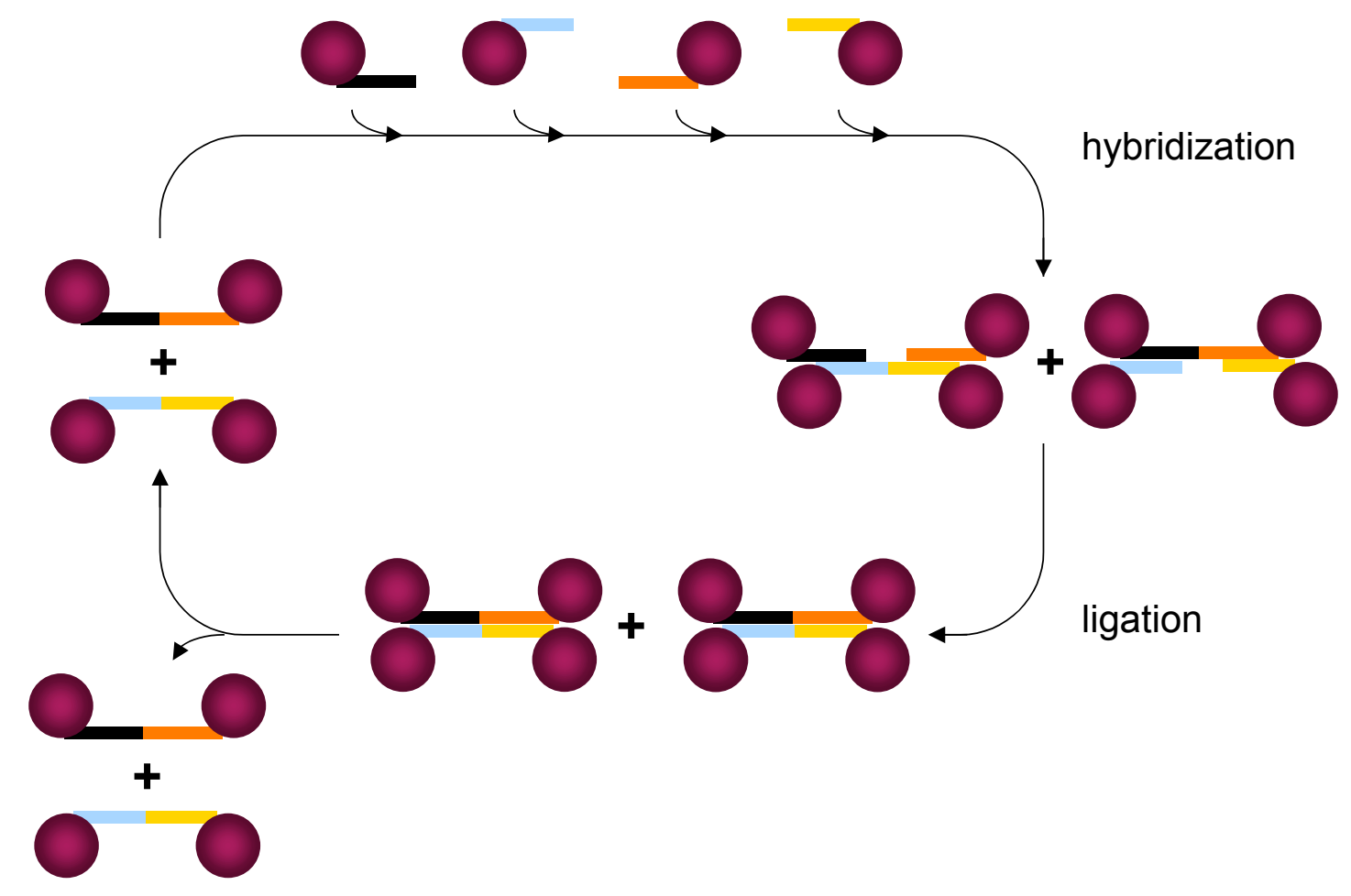

Figure 6.1 Schematic for amplification of dimers of gold nanocrystals linked with single-stranded DNA. Different colors refer to various DNA strands: black for dimer 1 strand $A$, orange for dimer 1 strand $B$, blue for dimer 2 strand B' and yellow for dimer 2 strand A'. Template strands with no gold nanocrystals are used in a first cycle to provide the template dimers for this schematic. 


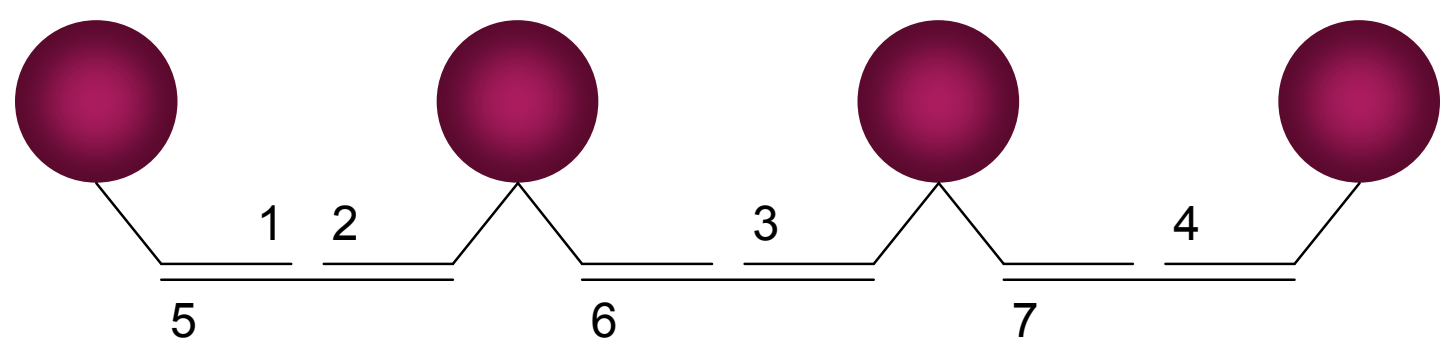

6.2 Tetramer for nano-amplification. Numbers refer to particular DNA strands. 
this approach, difunctionalized particles would be achieved either through the use of a DNA sequence incorporating a thiol group in the center of the strand, through attachment of a branched DNA molecule with two arms available for hybridization to templates, or through attachment of two DNA strands to the gold nanocrystal.

Amplification of nanostructures is desirable for several reasons. First, it can be used to preferentially purify the predominant nanostructure in a solution. This is a good way to amplify signal-in this case the most predominant nanostructure-from the noise-in this case the error structures. This method could be utilized for read-out of DNA computations using nanocrystals as markers for particular sequences of DNA. Secondly, if it were possible to conduct the amplifications in high yield, it would facilitate isolation of large amounts of purified nanostructures. Typically, electrophoresis is required to purify the Au/DNA conjugate building blocks, and then again to purify the resulting nanostructures. If a large amount of nanostructure is required, it is frequently necessary to run up to 50 gels, a labor-intensive process. By synthesizing a small amount of structure and subsequently amplifying, it may be possible to significantly reduce the number of gels required.

While the ultimate goal of nanostructure amplification has not yet been realized, significant progress has been made in illuminating the many issues that must be considered in such a complex system. These include the stability and surface 
characteristics of the gold nanocrystals, the accessibility of the DNA, and the effect of the buffer and presence of the gold nanocrystals on the activity of the DNA ligase. Reaction conditions for the ligation steps have also been investigated.

\section{Experimental Methods}

The gold nanocrystals I used in these experiments were $10 \mathrm{~nm}$ in diameter and were purchased from Ted Pella (Redding, CA). Several passivation and DNA conjugation methods were used. For the DNA-coated gold, the gold nanocrystals were first coated with bis-(p-sulfonatophenyl)phenyl phosphine (100 $\mathrm{mL}$ of approximately $5 \mathrm{nM}$ gold nanocrystals were stirred overnight with $30 \mathrm{mg}$ of the phosphine). These nanocrystals were then precipitated using a minimal amount of $\mathrm{NaCl}$ and centrifuged. The supernatant was removed and the particles were resuspended in distilled water. The precipitation-centrifugation steps were repeated as necessary. Discrete gold nanocrystal/DNA conjugates were then synthesized by incubation with thiolated DNA for two hours. The optimal ratio of DNA:gold nanocrystals was determined by a running a titration gel of conjugates synthesized using ratios ranging from 0.5 to 3 . The conjugates were purified using agarose gel electrophoresis $(3 \%, 0.67 \mathrm{~V} / \mathrm{cm}, 1 \mathrm{hr})$. The conjugates were removed from the gel using the standard procedure (see Chapter III), and they were then washed and concentrated using Millipore Microcon 50 centrifuge filters (Billerica, MA). DNA sequences were as follows: 
DNA strand for gold nanocrystal passivation, 5'-

XTTTTTTTTTTTTTTTTTTTTTTTTTTTTTT-3' (X = 5'-thiol modifier), dimer 1 strand A, 5'-

XTTTTTTTTTTTTTTTTTTTTTTTTTTTTTTGATGTTTACGCTTAGGAAGA-3' (X = 5'-thiol modifier), dimer 1 strand B, 5'-

YGCCAGTGTATCGCAATGACGTTTTTTTTTTTTTTTTTTTTTTTTTTTTTTX-3'

( $X=$ 3'-thiol modifier, $Y=5^{\prime}$-phosphorylate modifier), dimer 2 strand A', 5'-

XTTTTTTTTTTTTTTTTTTTTTTTTTTTTTTCGTCATTGCGATACACTGGC-3' (X = 5'-thiol modifier), dimer 2 strand B', 5'-

YTCTTCCTAAGCGTAAACATCTTTTTTTTTTTTTTTTTTTTTTTTTTTTTTTTTX-3' (X = 3'-thiol modifier, $Y=$ 5'-phosphorylate modifier), 24-base template, 5'CGATACACTGGCTCTTCCTAAGCG-3', and 40-base template, 5'CGTCATTGCGATACACTGGCTCTTCCTAAGCGTAAACATC-3'.

For the chemical ligation experiments, all procedures for passivation and conjugate synthesis were identical to those in Chapter III, though the DNA had several differences. The 5'-thiolated DNA had a 3'-phosphorylation modification and the 3'-thiolated DNA had a 5'-amino-T modification. DNA for this experiment was purchased from Synthegen (Houston, TX). DNA sequences were as follows: dimer 1 strand A, 5'-

XTTTTTTTTTTTTTTTTTTTTTTTTTTTTTTTTTGATGTTTACGCTTAGGAAGAY-3' (X = 5'-thiol modifier, $Y=3^{\prime}$-phosphorylate modifier), dimer 1 strand $B, 5$ 'YCCAGTGTATCGCAATGACGTTTTTTTTTTTTTTTTTTTTTTTTTTTTTTTTTX-3' (X 
= 3'-thiol modifier, $Y=5$ '-amino-T modifier), dimer 2 strand A', 5'-

XTTTTTTTTTTTTTTTTTTTTTTTTTTTTTTTCGTCATTGCGATACACTGGAY-3' (X

= 5'-thiol modifier, $Y=3$ '-phosphorylate modifier), dimer 2 strand B', 5'-

YCTTCCTAAGCGTAAACATCTTTTTTTTTTTTTTTTTTTTTTTTTTTTTTTTX-3' (X = 3'-thiol modifier, $Y=5$ '-amino-T modifier), EDC dimer template, 5'CGATACACTGGATCTTCCTAAGCG-3'.

For the experiments with 1.4-nm gold nanocrystals, six nmol of the maleimidefunctionalized 1.4-nm nanocrystals (Nanoprobes, Yaphank, NJ) were resuspended in $0.2 \mathrm{~mL}$ water. Thiolated DNA was reacted for two hours at room temperature with the nanocrystals, and excess DNA was washed away using Millipore Microcon 10 centrifuge filters. This step had a very low yield. The DNA sequences were the same as for the DNA-coated gold nanocrystals.

For the experiments using polyethylene glycol (PEG)-coated gold, 10-nm gold nanocrystals were first incubated with DNA in the optimal ratio to obtain 1:1 DNA:gold nanocrystal conjugates. Then, before electrophoresis, the conjugates were incubated with a 500-fold excess of the PEG of interest for two additional hours. These conjugates were then concentrated by high-speed centrifugation (32,000 rpm in an Eppendorff microcentrifuge). After concentration to $\sim 0.5 \square \mathrm{M}$, these conjugates were purified using agarose gel electrophoresis. For gold nanocrystals passivated with smaller PEG molecules, purification was done using the standard $3 \%$ gels, while for gold nanocrystals passivated with the 
larger PEG molecules, purification was done using $1 \%$ gels. DNA sequences were the same as for the DNA-coated gold experiments.

T4 DNA ligase from New England Biolabs (NEB, Beverly, MA) was used for all enzymatic ligation experiments. For synthesis of dimers, $1 \mathrm{LL}$ of 400,000 units/mL ligase was incubated with $20 \square \mathrm{L}$ of $1 \square \mathrm{M}$ gold nanocrystal/DNA conjugates for 20 minutes to 2 hours at a temperature of $16-25^{\circ} \mathrm{C}$. Ligase buffers were prepared according to manufacturer recommendations, except DTT, detergents, and BSA were left out. In addition, the concentration of $\mathrm{Mg}^{2+}$ was varied to determine the minimum effective concentration. After ligation, samples were concentrated using Microcon 50 centrifuge filters (for 10-nm nanocrystals) or Microcon 10 centrifuge filters (for 1.4-nm nanocrystals). The samples were then characterized using gel electrophoresis. The ligase was deactivated either by incubation at $65^{\circ} \mathrm{C}$ or by mixture with the EDTA present in the electrophoresis buffers (EDTA chelates all the $\mathrm{Mg}^{2+}$ in the solution, which is necessary for ligation). The EDTA method was superior to the heat method, since heat can promote nanocrystal aggregation. Post-ligation samples were occasionally characterized using TEM.

For chemical ligation experiments, EDC-HCl was purchased from Aldrich (St. Louis, MO). The buffer used was 0.1 M HEPES or 0.1 M HEPES together with $0.5 x \mathrm{TBE}$. The concentration EDC- $\mathrm{HCl}$ used in these experiments was $0.1 \mathrm{mM}$, as higher concentrations of EDC caused precipitation of the gold nanocrystals. 
Gold nanocrystal concentrations were typically $0.2 \square \mathrm{M}$, as this was a typical concentration after isolation of the pure conjugates. Ligations were run for $6 \mathrm{hrs}$ at temperatures of $25-60{ }^{\circ} \mathrm{C}$. The samples were then concentrated and characterized using gel electrophoresis. Concentration was done via heated vacuum centrifugation or centrifuge filtration.

Amplifications were done by cycling the ligase/conjugate solutions in a thermal cycler. Ligation segments were typically interspersed with a 5- to 10-minute dehybridization period at a temperature between 50 and $60^{\circ} \mathrm{C}$. This temperature was chosen based on the melting temperature of the melted single-strand dimmers as well as the deactivation temperature of the T4 DNA ligase. A higher temperature was desirable for dehybridization, while a lower temperature was desirable for gold nanocrystal and ligase stability. While Taq DNA ligase was tested for suitability in ligations and amplifications of gold nanocrystal/DNA conjugates and dimers, no conditions were ever found that allowed function of the ligase and stability of the gold nanocrystals simultaneously.

Controls were run for all experiments. The most important were controls with all reaction components except the gold nanocrystals. In these cases, the DNA was not thiolated. Controls were also run where template DNA, ligase buffer and/or ligase was left out of the sample. The length of DNA template used was also varied, as were reaction times, temperatures, and concentration methods. 


\section{Results}

As synthesized, gold nanocrystals are typically coated with citrate ions. While these nanocrystals are stable in pure water, repelling each other on the basis of charge and thus avoiding aggregation due to van der Waals attraction, they are not sufficiently stable to withstand the decreased double layer thickness in buffer solutions with high ionic strength. For synthesis of the dimers and trimers of nanocrystals discussed in previous chapters, the gold nanocrystals are generally coated with bis-(p-sulfonatophenyl)phosphine ligands. This phosphine yields a surface more resistant to aggregation in higher ionic strength solutions. As a result, nanocrystals are stable under the salt conditions required for DNA binding and hybridization as well as the buffers used for electrophoresis.

Many biological enzymes perform optimally in buffers containing a number of chemicals that have destabilizing effects on gold nanocrystals. These include dithiothreitol (DTT), $\mathrm{Mg}^{2+}$, detergents, and other ionic substances. As a dithiol, DTT causes quick and irreversible aggregation of the gold nanocrystals through cross-linking. Since the ionic strength is proportional to the square of an ion's charge, $\mathrm{Mg}^{2+}$ has a stronger effect on the ionic strength of the solution than a monocation, and so the electrical double layer is decreased by a factor of 2 for the same concentration of ion. This can be understood by examining the equations for ionic strength and the double layer thickness: 


$$
\begin{gathered}
1 / \square=\left(\left[R T / F^{2} I\right)^{1 / 2}\right. \\
I=1 / 2 \square z_{i}^{2} m_{i}
\end{gathered}
$$

where $1 / \square$ is the double layer thickness, $\square$ is the electric permittivity of the solvent, $\mathrm{R}$ is the gas constant, $\mathrm{T}$ is the absolute temperature, $\mathrm{F}$ is the Faraday constant, I is the ionic strength, $z_{i}$ is the charge of the ith ion, and $m_{i}$ is the concentration of the ith ion. Detergents are frequently zwitterionic, and so have an effect similar to the dication. As a result of these effects, the phosphine generally used is not sufficient to stabilize the nanocrystals in the buffers needed for optimal ligase activity.

Several approaches to avoiding aggregation were investigated. In one, DNA was used to coat the gold nanocrystals. In another, non-enzymatic chemical ligation was attempted, using mono-functionalized 1.4-nm gold nanocrystals. In a final method, thiolated polyethylene glycol (PEG) molecules were used to passivate 5and 10-nm nanocrystals.

\subsection{Nanocrystal Surface Passivation with DNA}

This approach draws on previous experiments in which gold nanocrystals were coated with DNA used to link the nanocrystals [28]. In these experiments, particles were stable under high salt conditions. Two key issues in this strategy are the DNA sequence length and the sequence itself. Previous results from Parak et al., indicate that passivation with DNA sequences from 8 to 30 bases in 
length results in a dense layer of DNA oriented perpendicular to the particle surface [15]. Additionally, Mirkin et al. determined that T has a lower affinity for gold surfaces than any of the other nucleotide bases [34]. Thus, oligonucleotides 5'-HS- $(T)_{n}$ with $n$ ranging between 8 and 30 were screened for utility as surface ligands. Passivation with 5'-HS-(T) $)_{30}$ resulted in nanocrystals with a very high tolerance for salts, up to $500 \mathrm{mM}$ in $\mathrm{Mg}^{2+}$ and up to saturated $\mathrm{Na}^{+}$. These nanocrystals were also stable in the buffers typically used for ligation.

Although particles coated with long thiolated DNA were stable in ligation buffer, they also presented difficulties in attaching the thiolated DNA sequence used for ligation. In order to attach the DNA needed for the ligation, several methods were investigated. The most successful of these methods was to purify gold/DNA conjugates prior to coating the gold with thiolated poly-T DNA. The DNA for ligation was 50 bases in length and thiolated on either the $3^{\prime}$ or 5 ' end. The 30 bases nearest to the thiol were $T$, similar to the passivating strands. The next 20 bases were complementary to the template that would hold the two conjugates together while the ligase linked them. After the conjugates were purified, they were incubated with approximately a 100-fold excess of the 5'-HS$(T)_{30}$ DNA. Conjugates were incubated overnight and were then concentrated using a one of several methods, including centrifugal filtration or vacuum centrifugation. In general, the vacuum centrifugation was found to be the better method, since the increasing ionic strength in such a circumstance greatly increases the density of DNA it is possible to attach to a gold nanocrystal [28]. In 
this method, $\mathrm{NaCl}$ concentrations began at $10 \mathrm{mM}$ and eventually rose to approximately $1 \mathrm{M}$. The disadvantage of this method was that subsequent washing and centrifugal filtration was required to reduce the ionic strength; since each step increases the risk of aggregation, it is desirable to minimize the number of steps in the experiments.

When the concentrated, passivated nanocrystals were then introduced to ligase and/or ligase buffers, dimers of gold nanocrystals were produced regardless of other conditions, including presence of the other type of conjugate or the connecting template strand. It was eventually determined that DNA passivation was not suitable for ligation experiments. This prompted the investigation of other passivating agents and ligation protocols.

\subsection{Chemical Ligation of Gold Nanocrystal/DNA Conjugates}

Chemical ligation was developed by researchers interested in the chemical origins of life. For this and other reasons, researchers have used chemical ligation techniques to amplify manipulate and even amplify DNA in a manner analogous to polymerase chain reaction [108-109]. Here, chemical ligation was attempted using 5-nm gold nanocrystal conjugates passivated with bis-(psulfonatophenyl)phenyl phosphine dihydrate dipotassium salt. Chemical ligation can be carried out in buffers that are more compatible with the gold nanocrystals. 
Also, since enzymes are often quickly degraded and are very sensitive to environment, it was thought that chemical ligation could result in a more robust system.

The reaction mechanism of chemical ligation is shown in Figure 6.3. An oligonucleotide with a 3'-phosphorylated adenine was annealed with an oligonucleotide with a 5'-amine modified thymine onto a template strand. N-(3dimethylaminopropyl)-N'-ethylcarbodiimide hydrochloride (EDC) was then allowed to react with the DNA, linking the two strands. This method of chemical ligation was chosen over others [108-114] because the linkage has the same size as the normal sugar-phospate backbone, allowing optimal hybridization in repeated cycles of ligation. Other methods of chemical ligation insert multiple atoms in the place of one of the phosphate oxygen atoms [110-114]. Unfortunately, in this system chemical ligation was found to be prohibitively lowyielding, again leading to consideration of alternative protocols.

\subsection{4-nm Mono-functionalized Gold Nanocrystals}

The next protocol investigated began with 1.4-nm mono-functionalized gold nanocrystals. While smaller gold nanocrystals lack some of the benefits (such as strong surface plasmons) associated with larger particles, mono-functionalized particles of this size are commercially available, eliminating several steps from the experimental procedure. 


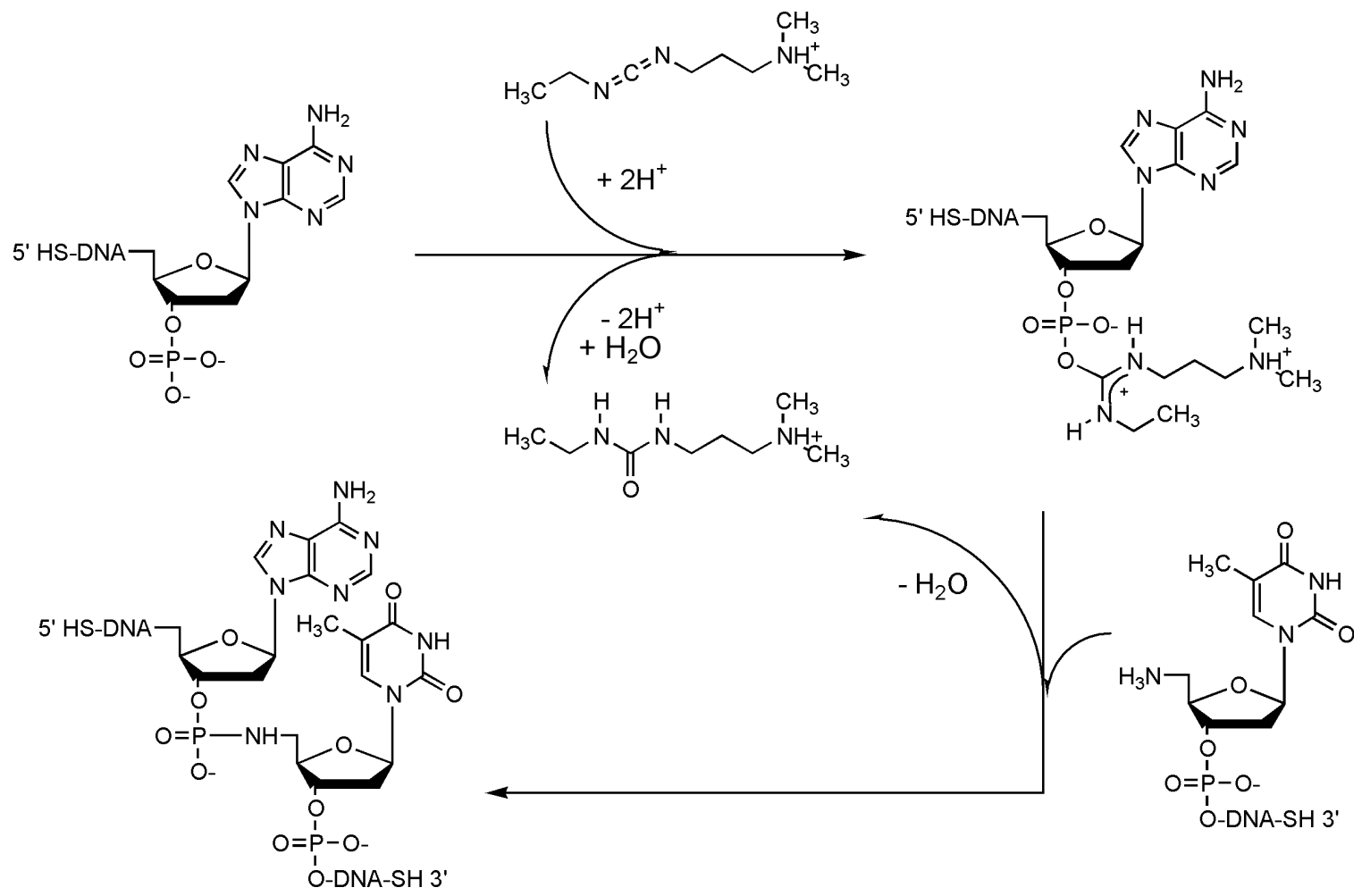

Figure 6.3 Chemical ligation with EDC. 
There are several advantages to using mono-functionalized gold nanocrystals for these experiments. First of all, it should not be necessary to do additional passivation of the gold surfaces [51]. Secondly, it becomes unnecessary to separate the conjugates with a single DNA strand from the initial conjugate mixture, saving the laborious work of the electrophoretic purification. Third, while these nanocrystals do not exhibit the red color of the larger nanocrystals, it is still possible to image them using white light and to separate them using gel electrophoresis (Figure 6.4). Their main disadvantages are their lack of the red color, the extreme difficulty of imaging them in standard resolution TEMs, their relatively lower stability in solution, and the fact that it is not possible to make multiple-DNA conjugates using these nanocrystals.

Through the use of the 1.4-nm monofunctionalized nanocrystals, the initial steps of nanocrystal passivation and concentration were eliminated, as was the conjugate purification step.

Attachment of DNA to the nanocrystals was performed according to the supplier's instructions. Six nmol of the maleimide-functionalized 1.4-nm nanocrystals (Nanoprobes, Yaphank, NJ) were resuspended in $0.2 \mathrm{~mL}$ water. The nanocrystals were resuspended immediately before use to avoid deactivation of the maleimide functional group through hydrolysis. Thiolated DNA was incubated with the gold nanocrystals for 2 hours at room temperature 


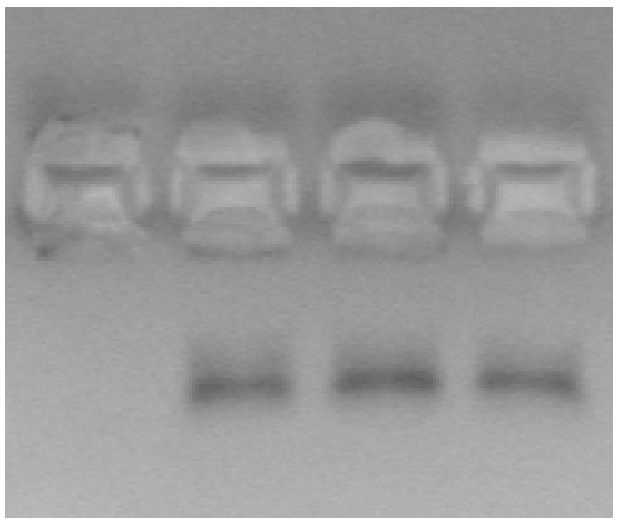

Figure 6.4 Electrophoresis of 1.4-nm gold nanocrystal/DNA conjugates. From left to right, the first lane is 1.4-nm gold nanocrystals with no DNA (since it is neutral it stays in the well), the second lane is the nanocrystals reacted with a 1:1 ratio of DNA:gold nanocrystals, the third corresponds to a 3:1 ratio of DNA:gold nanocrystals, and the fourth corresponds to a 5:1 ratio of DNA:gold nanocrystals. 
or overnight in the refrigerator. The remainder of the procedure is identical to that for the DNA-coated gold particles discussed in section 3.1.

After the attachment of DNA, which was done at low concentration, according to manufacturer instructions, it was necessary to concentrate the nanocrystal solution. Vacuum centrifugation, centrifugal filtration, and dialysis techniques were utilized. Use of centrifuge filters resulted in a high degree of sample loss. Vacuum centrifugation proved infeasible due to the dissociation of the passivation layer at elevated temperature. Finally, low-volume dialysis tubes were used to reduce the ionic strength of the conjugate solution prior to concentration by one of the methods discussed previously. In this case, although the dialysis was successful, concentration still resulted in an unacceptable degree of sample loss.

\subsection{PEG-Coated Gold Nanocrystals}

The highest degree of success was achieved using nanocrystals coated with strands of thiolated polyethylene glycol. Polyethylene glycol (PEG) is available in a great variety of lengths and functionalities, even with dual functionalities such as thiol and carboxylic acid groups. PEG is also very inert in biological environments, and does not interfere with enzymatic and cellular processes [115]. Since the PEG chain itself is charge-neutral, it can be used to minimize electrostatic interactions between nanocrystals or biomolecules. In addition, long 
PEG chains are very effective at screening van der Waals interactions between nanocrystals.

Initially, short thiolated PEG molecules were used (PEG acid thiol, MW 458.6 g/mol, Polypure, Oslo, Norway). A shorter strand would allow maximal access to the DNA strand needed for ligations, while still affording the stabilization required from the passivating layer. It was found that it was still possible to purify the nanocrystals using electrophoresis after coating with PEG, a significant advantage over the DNA-coated nanocrystals. Nonetheless, these nanocrystals also exhibited site-specific aggregation in the presence of ligase buffers and thermal cycling (Figure 6.5). In this case, however, the aggregation was much more controlled than with the DNA-coated nanocrystals, and only occurred when the nanocrystals were attached to at least one DNA strand. It was found that dimers of gold nanocrystals were formed with in all conditions where salt temperature, enzymes or buffers of any kind were involved. This was called 'site-specific aggregation.'

This problem was eliminated when longer strands of PEG were used (thiol terminated polyethylene oxide, MW $2100 \mathrm{~g} / \mathrm{mol}$, Polymer Source, Montreal, Canada). Several end-terminal groups were used: $-\mathrm{OH},-\mathrm{COOH}$, and $-\mathrm{OCH}_{3}$. These nanocrystals did not undergo site-specific aggregation; ongoing experiments in the group will focus on verifying whether ligation with these conjugates is possible. These PEG-coated nanocrystals with no DNA attached 


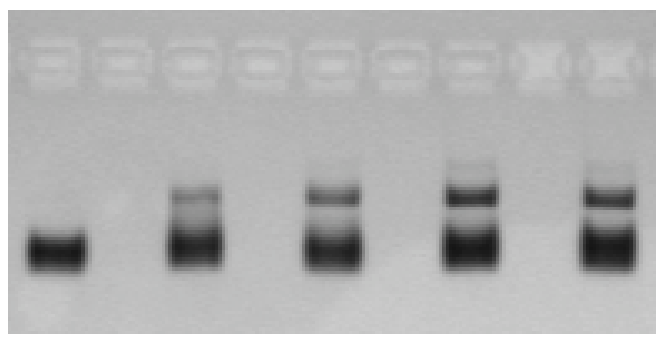

Figure 6.5 Results of a ligation experiment using 5-nm gold nanocrystals passivated with short, $-\mathrm{COOH}$ terminated PEG ligands. The first lane shows PEG-coated $10 \mathrm{~nm}$ gold nanocrystal/50b DNA conjugates, the second shows conjugates with ligase buffer and $40 \mathrm{~b}$ template, the third shows conjugates with ligase and $40 \mathrm{~b}$ template, the fourth shows conjugates with ligase, ligase buffer and $24 \mathrm{~b}$ template, and the fifth shows conjugates with ligase, ligase buffer and $40 \mathrm{~b}$ template. Only the fourth and fifth lanes had the required materials for ligation. Site-specific aggreagation was determined to be the cause of the slower band in the second to fifth lanes. 
were uncharged, so they did not migrate in the gels. As more DNA was added, the nanocrystals moved more rapidly in the gel, since the size was relatively unchanged and the average surface charge of the nanocrystals were increasing with each added DNA strand (Figure 6.6).

\section{Conclusion}

Enzymatic manipulation has tremendous potential utility in the construction of nanocrystal/biomolecule hybrid materials. While several examples have shown use of enzymes in the manipulation of gold nanocrystals coated with DNA, it appears that discrete systems are more sensitive and require extensive optimization of experimental conditions. Nonetheless, amplification of discrete nanostructures is an exciting goal, and the experiments presented here address many of the key concerns in developing a successful conjugate amplification system. 


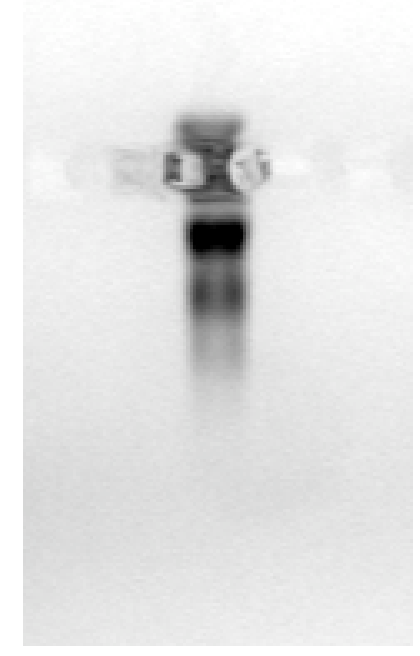

Figure 6.6 Gold nanocrystal/DNA conjugates stabilized with long -OH terminated PEG ligands. The band just above the well corresponds to gold nanocrystals with no DNA attached, the darkest band (just below the well) corresponds to gold nanocrystals with one 50b DNA strand attached, and each successive lower band corresponds to an additional strand of DNA attached to the gold nanocrystal. 


\section{Chapter VII}

\section{Conclusions}

\section{Biomolecular Assembly of Gold Nanocrystals}

As a result of the work presented in this dissertation, more is known about the characteristics of nanostructures constructed from gold nanocrystals and DNA. Electrophoretic techniques were developed, understanding of the mechanisms of electrophoresis for nanocrystal/DNA conjugates was gained, and a more quantitative understanding of TEM data was attained. In addition, new information about passivation of gold nanocrystal surfaces was discovered and the issues involved in using biological enzymes with discrete nanocrystal/biomolecule hybrid materials were described.

As always, any body of research serves primarily as the starting point for new study, and is successful in the extent to which that occurs. While the next steps entailed for many of the projects I completed are already in progress or are finished, in the next section, I will review the prospects for research in the area of synthesis and characterization of discrete nanocrystal/biomolecule conjugates and nanostructures as a whole. 


\section{Prospects for Discrete Nanostructures}

\subsection{Options for New Synthesis}

Nanocrystal/biomolecule conjugates are synthesized by mixing chemically activated biomolecules with appropriately derivatized, water-soluble nanocrystals. Under these conditions, both nanocrystals and biomolecules may retain at least some of their properties and/or bioactivity. In addition to conventional nanocrystals, some researchers have extended their work into fullerene materials, polymers, and protein/DNA conjugates [116-119]. In an interesting example, Mann and coworkers biotinylated horse spleen ferritin, a protein that contains a nanocrystal of ferric oxide approximately $5 \mathrm{~nm}$ in diameter [118]. Streptavidin was then used to crosslink the ferritin. In another example, mentioned previously, Niemeyer and coworkers synthesized and isolated discrete streptavidin/DNA conjugates [119]. These additional structures could serve as useful components in nanocrystal/biomolecule hybrid structures and their applications.

RNA and peptide nucleic acid are logical extensions of work with DNA.

PNA/DNA helices are energetically more stable than DNA helices, and, as a result, PNA is much less likely to dehybridize in cellular conditions than DNA. Thus, it may be a good option for in situ experiments. PNA/DNA hybrids have already been conjugated to gold nanocrystals [120]. RNA has been used to 
make a number of nanostructures such as triangles and 3D arrays [121]. Each of these methods can be extended for use in discrete nanostructures.

Use of biological enzymes provides a logical extension for the synthesis and manipulation of nanocrystal/biomolecule nanostructures. Additional work has been done using gold nanocrystals coated with DNA that could be extended to discrete structures. Wang et al. cut DNA attached to gold nanocrystals with restriction enzymes [122], Kanaras et al. ligated and cut DNA attached to gold nanocrystals with DNA ligase and restriction enzymes [28], and Nicewarner Peña et al. used DNA polymerase to extend DNA fragments attached to gold nanocrystals [107].

Another interesting avenue of work involves the association of nanocrystals and proteins. Antibody-antigen-assembled networks of gold and silver nanocrystals have been fabricated [95], and biotin-streptavidin recognition-assembled networks of gold nanocrystals have been made by connecting streptavidincoated gold with a dibiotinylated DNA linker [94]. Protein assembly provides several important advantages for nanocrystal assembly, which has been discussed in a review by Mann et al.: protein assembly has already been widely used in diagnostics, proteins have potential for activity in multiple solvents, and there are many different non-overlapping sets of proteins that assemble. Nonoverlapping sets of proteins include pairs such as biotin-avidin and antibodyantigen pairs. The proteins in these pairs interact with one another but not with 
proteins in the other pair. Conversely, great care must be taken to design DNA sequences that do not contain stable secondary structures or segments that hybridize with the wrong strands or locations.

More complex DNA interactions provide expanded capabilities for producing nanocrystal/DNA structures. The structural transitions of DNA can be used to create micromechanical devices, and several groups have provided examples of this. In one, Seeman and coworkers created a device utilizing the B-Z transition of DNA [123]. In another, G-G mismatch sequences, G-quartets, and the unique structural transitions these create were utilized [124]. G-quartets can also be used as a connection point for two- and three-dimensional assembly of DNA and nanocrystal/DNA hybrid materials. Seeman uses immobile double and triple crossover analogues to Holliday junctions, observed in replicating DNA. Fahlman and Sen use the very stable G quartet to create side-by-side "synapses" between double-stranded DNA [125]. They can achieve selfspecificity by altering the sequence of the G-G mismatch sections by adding T-T mismatch sequences at selected points. Changes in DNA conformation have also been used to bring together reactants for chemical reactions. This could be expanded to select between several different reactions, depending on the state of the surroundings [126].

Tobacco mosaic virus (TMV) has also been used to create a potentially addressable nanocrystal/biomolecule material [127]. Platinum, gold, and silver 
nanocrystals have been used to decorate the outsides and insides of TMV. This is actually an example of biomolecular templating, because the nanocrystals are synthesized on the virus surface and in the tube-shaped interior of the virus. Creating linear, straight, rigid wires inside TMV and then attaching the ends of the TMV to some other nano- or bio-material could yield an interesting way to produce self-assembling interconnects for nano-electronic components.

\subsection{Options for New Applications}

There are many possibilities for the application of nanocrystal/biomolecule conjugate materials in materials science as well as biology. The most prominent of these possibilities is in the area of nanoelectronics, which will be discussed. Molecular computing could also make parallel computing with an arbitrary and virtually unlimited number of variables a reality.

In addition, there are possibilities for many new bioassays. One of particular interest is the use of DNA enzymes, also known as DNAzymes. In one example, $\mathrm{Pb}^{2+}$ ions activate a DNAzyme to restrict DNA at a particular sequence of DNA. The DNA to be cut is the DNA linking gold nanocrystals into a network, which has a characteristic blue color; when the DNAzyme cuts the DNA, the gold nanocrystals are released and a bright red color is restored to the solution [128129]. 


\subsubsection{Bio-Nano-Electronics}

Eichen et al., enumerate three challenges that scientists need to address before electronics can be miniaturized using a bottom-up approach: positioning of components, wiring of components, and interfacing the nanocomponents with the macro world [130]. While the approach they took did not make use of discrete nanocrystal/biomolecule materials, many other researchers are exploring the possibilities of these materials for use in nanoelectronics.

Nanoscale component positioning on stretched DNA has been shown by Keren et al., who used DNA/protein conjugate structures to attach single-walled nanotubes (SWNT) at specific locations of a larger DNA substrate [131]. RecA single-strand DNA (ssDNA) binding protein binds to a fragment of ssDNA. This material is then bound to a conjugate sequence on a template dsDNA. Antibody interactions are used to connect SWNT to the RecA/dsDNA structure, and the remaining dsDNA is metallized by first using silver reduction to create seed nanocrystals onto which gold can reduce to form nanowire connections. On the proper substrate, this nanostructure functions as a field-effect transistor. Work such as this follows the same vein as discrete nanocrystal/biomolecule conjugate work since it uses biological materials to specifically address inorganic nanomaterials, creating a highly parallel method of creating discrete, ordered hybrid materials. 
It is also possible to arrange inorganic nanocrystals or biomolecules on DNA in a non-sequence-specific manner. Currently, many of these applications do not use discrete conjugates; nonetheless, the resulting nanostructures are not highly cross-linked due to the immobilization of the template DNA. While use of discrete conjugates would enable decoration of long DNA in solution, thus enabling solution-only applications, this non-discrete, surface based method eliminates the need for time-consuming purification of the nanomaterial/biomolecule conjugates. In one example, $\mathrm{C}_{60}$ molecules were arranged on plasmid DNA, a surprisingly good contrast agent for biological electron microscopy [132]. In another, streptavidin-coated gold nanocrystals were arranged on long, stretched DNA by associating them with short, biotinylated oligonucleotides hybridized to the long DNA [133], and in another example, $(A T)_{n}$ coated gold nanocrystals were used to decorate long DNA strands at sequence-specific locations [134]. The spacing between nanocrystals can be carefully controlled by using coatings on the gold surface with a specific radius [135].

There are several approaches being explored for component wiring in nanoelectronics. One method employs structural transitions of DNA to construct nanowires: results showing that $\mathrm{Au}_{55}$ clusters arrange themselves along the major groove of B DNA have been published [50]. When the B to A DNA transition occurs, the gold clusters become $\mathrm{Au}_{13}$ clusters due to the shrinkage of the major groove. It is remarkable that a biomolecular transition such as this one 
can change the gold nanocrystals to this extent. $\mathrm{Au}_{55} / \mathrm{DNA}$ interactions may be shown to possess cytotoxic capabilities that could be utilized in anti-cancer treatments [50].

An important goal for nanoelectronics is the creation of nanocrystals with not only a discrete number of functionalizations, but also defined angular attachment points. Controlled angular placement of biomolecules on nanocrystals could also provide predictable nanostructure conformation, maximal enzymatic activity, maximal yields for DNA hybridizations, and optimal efficiency for energy transfer. Tetravalent colloids may be especially useful, since it is predicted that is a fourcoordinate colloidal crystal of nanocrystals with a high dielectric contrast with the surrounding material should have a large photonic band-gap. Nelson suggests several ways to control angular attachment of functional groups to spherical nanocrystals [136]. These possible functional groups include anisotropic triblock copolymers, gemini lipids, and inorganic nanorods.

In addition, several groups have improved the process of stretching DNA on surfaces, something that will be useful for creating electronic circuitry, as well as sequence detection and enzymatic activity studies [137]. Stretching has been realized using solvent evaporation and poly-L-lysine on positively charged mica surfaces [138] and through the use of kinesin [139]. Stretched DNA arrays have been used as masks for molecular lithography. The DNA structures assemble in 
solution and are deposited on a substrate. Metal is then evaporated over the DNA mask, which is then removed, revealing the negative image.

\subsubsection{Molecular computing}

Gold nanocrystals may also be used to improve the speed and efficiency of information-reading steps in DNA computing [140] and quantum computing [141], two promising routes to realizing parallel-processing computers that can easily solve np-complete problems. The linear arrangement of nanocrystals at appropriate spacings could enable the coding of bits of information along the array. This has already been done using fluorescent tags instead of nanocrystals [142], though the advantages and versatility of nanocrystals would be a great addition. While DNA carries information in its sequence, this information can often be difficult to read, requiring lengthy, tedious isolation, cloning, PCR, and sequencing steps. For applications such as DNA computing, when it is desirable to obtain information from DNA in a much more rapid fashion, techniques such as those developed by Reif and coworkers could be extremely useful. Reif and coworkers have devised a method to take a DNA template strand encoding a nano-barcode and use it to construct a quadruple crossover molecule that is decorated with DNA stem loops in the 1 positions (Figure 7.1) [49]. This produces a highly accurate, easy-to-read nano-barcode. In addition, extension of this patterning technique could be useful in microfabrication and other applications requiring arrangement of nanocrystals, proteins or molecules. 
a
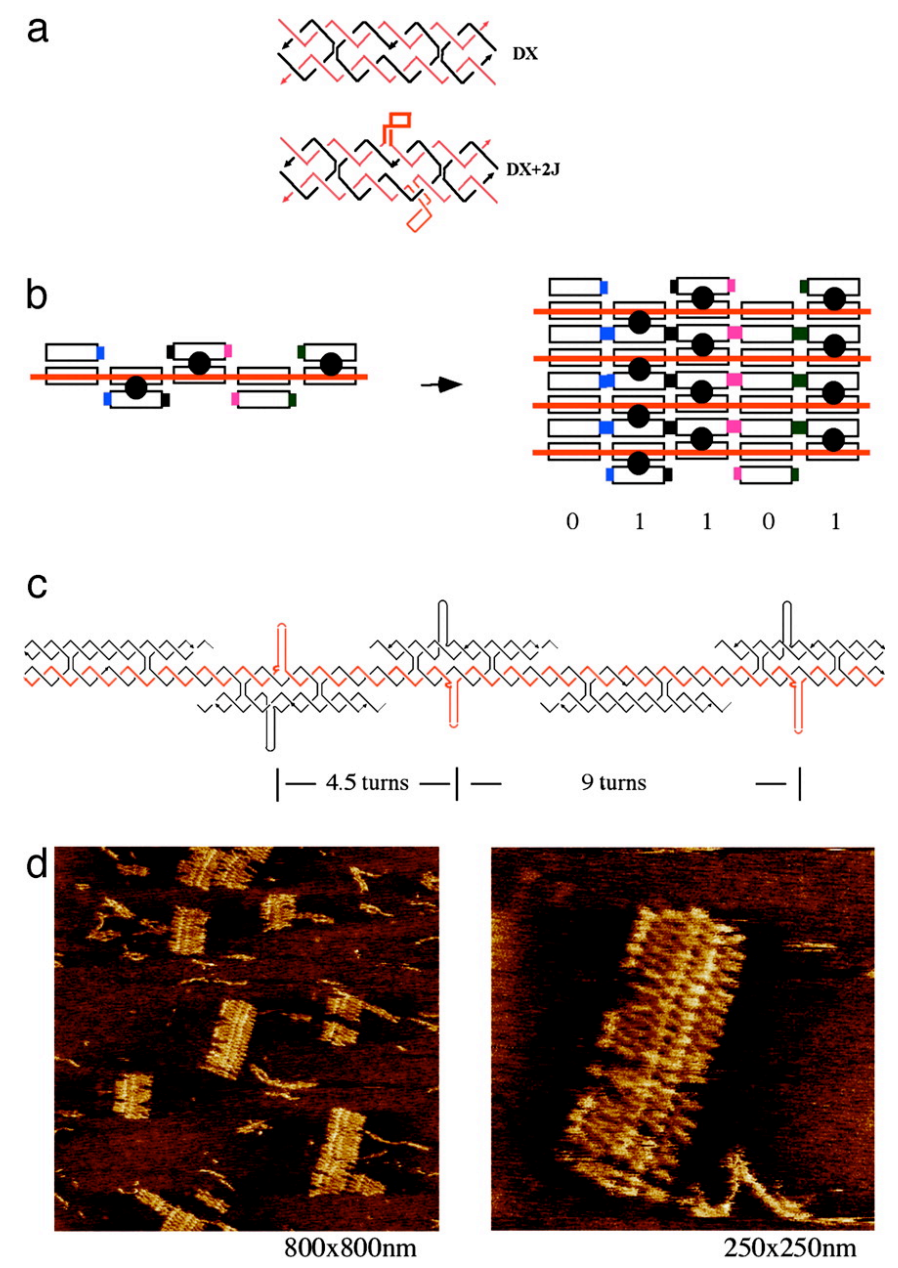

Figure 7.1 (a) Line drawing of the two DNA tiles used in the barcodes. Each has 5 DNA strands. (b) Schematic of self-assembly of barcode lattice layers based on DNA tiles around a scaffold strand. The barcode information (01101) is represented by either the presence (designated 1) or the absence (designated 0) of a stem loop (shown as a black circle) protruding out of the tile plane. (c) Strand structure of one barcode layer. (d) AFM visualization. The distance between the two closer adjacent stripes is $\sim 16 \mathrm{~nm}$, and the further adjacent stripes is $\sim 31 \mathrm{~nm}$. Reprinted with permission from Yan, H., LaBean, T.H., Feng, L.P. and Reif, J.H. PNAS 100, 8103-8108 (2003). 


\section{Conclusions}

As a result of the work I conducted for this dissertation, more is known about the synthesis and characterization of discrete 'nanocrystal molecules' of gold nanocrystals linked with DNA. As a result of work towards synthesis of nanostructures using DNA ligase, much more is known about passivation of gold nanocrystal surfaces for use in biologically sensitive environments. This work will continue to serve as a foundation for future synthesis and characterization of discrete nanocrystal/biomolecule nanostructures. 


\section{References}

[1] Mirkin, C.A., Letsinger, R.L., Mucic, R.C., and Storhoff, J.J. A DNA-based method for rationally assembling nanoparticles into macroscopic materials. Nature 382, 607-609 (1996).

[2] Daniel, M.C. and Astruc, D. Gold nanoparticles: assembly, supramolecular chemistry, quantum-size-related properties, and applications toward biology, catalysis, and nanotechnology. Chem. Rev. 104, 293-346 (2004).

[3] Fritzsche, W. and Taton, T.A. Metal nanoparticles as labels for heterogeneous, chip-based DNA detection. Nanotech. 14, R63-R73 (2003).

[4] Shenhar, R. and Rotello, V.M. Nanoparticles: scaffolds and building blocks. Accounts of Chemical Research 36, 549-561 (2003).

[5] Niemeyer, C.M. Nanoparticles, proteins, and nucleic acids: biotechnology meets materials science. Angew. Chem. Int. Ed. 40, 4128-4158 (2001).

[6] Katz, E. and Willner, I. Integrated Nanoparticle-Biomolecule Hybrid Systems: Synthesis, Properties, and Applications. J. Am. Chem. Soc. 43, 6042-6108 (2004). 
[7] Alivisatos, A.P., Johnsson, K.P., Peng, X.G., Wilson, T.E., Loweth, C.J., Bruchez, M.P., and Schultz, P.G. Organization of 'nanocrystal molecules' using DNA. Nature 382, 609-611 (1996).

[8] Claridge, S.A., Goh, S.L., Frechet, J.M.J., Williams, S.C., Micheel, C.M., and Alivisatos, A.P. Directed assembly of discrete gold nanoparticle groupings using branched DNA scaffolding. Chem. Mater. 17, 1628-1635 (2005).

[9] Pellegrino, T., Parak, W.J., Boudreau, R., Le Gros, M.A., Gerion, D., Alivisatos, A.P. and Larabell, C.A. Quantum dot-based cell motility assay. Differentiation 71, 542-548 (2003).

[10] Loweth, C.J., Caldwell, W.B., Peng, X.G., Alivisatos, A.P., and Schultz, P.G. DNA-based Assembly of gold nanocrystals. Angew. Chem. Int. Ed. 18, 1808$1812(1999)$.

[11] Zanchet, D., Micheel, C.M., Parak, W.J., Gerion, D., and Alivisatos, A.P. Electrophoretic isolation of discrete Au nanocrystal/DNA conjugates. Nano Lett. $1,32-35(2001)$.

[12] Zanchet, D., Micheel, C.M., Parak, W.J., Gerion, D., Williams, S.C., and Alivisatos, A.P. Electrophoretic and structural studies of DNA-directed Au nanoparticle groupings. J. Phys. Chem. B 106, 11758-11763 (2002). 
[13] Cha, J.N. and Alivisatos, A.P. Protein mediated assembly of asymmetric gold nanostructures. In preparation.

[14] Micheel, C.M., Zanchet, D., Parak, W., and Alivisatos, A.P. Hydrodynamic properties of Au/DNA conjugates. In preparation.

[15] Parak, W.J., Pellegrino, T., Micheel, C.M., Gerion, D., Williams, S.C., and Alivisatos, A.P. Conformation of oligonucleotides attached to gold nanocrystals probed by gel electrophoresis. Nano Lett. 3, 33-36 (2003).

[16] Sonnichsen, C., Reinhard, B.M., Liphardt, J., and Alivisatos, A.P. A molecular ruler based on plasmon coupling of single gold and silver nanoparticles. In preparation.

[17] Raschke, G., Kowarik, S., Franzl, T., Sonnichsen, C., Klar, T.A., Feldmann, J., Nichtl, A. and Kurzinger, K. Biomolecular recognition based on single gold nanoparticle light scattering. Nano Lett. 3, 935-938 (2003).

[18] Bruchez, Jr., M., Moronne, M., Gin, P., Weiss, S. and Alivisatos, A.P. Semiconductor nanocrystals as fluorescent biological labels. Science 281, 20132016 (1998). 
[19] Gerion, D., Pinaud, F., Williams, S.C., Parak, W.J., Zanchet, D., Weiss, S., and Alivisatos, A.P. Synthesis and properties of biocompatible water-soluble silica-coated CdSe/ZnS semiconductor quantum dots. J. Phys. Chem. B 105, 8861-8871 (2001).

[20] Fu, A., Micheel, C.M., Cha, J.N., Chang, H., Yang, H., and Alivisatos, A.P. Discrete nanostructures of quantum dots/Au with DNA. J. Am. Chem. Soc. 126, 10832-10833 (2004).

[21] Parak, W.J., Gerion, D., Zanchet, D., Woerz, A.S., Pellegrino, T., Micheel, C., Williams, S.C., Seitz, M., Bruehl, R.E., Bryant, Z., Bustamante, D., Bertozzi, C.R., and Alivisatos, A.P. Conjugation of DNA to silanized colloidal semiconductor nanocrystalline quantum dots. Chemistry of Materials 14, 2113$2119(2002)$

[22] Gerion, D., Parak, W.J., Williams, S.C., Zanchet, D., Micheel, C.M., and Alivisatos, A.P. Sorting fluorescent nanocrystals with DNA. J. Am. Chem. Soc. 124, 7070-7074 (2002).

[23] Gerion, D., Chen, F., Kannan, B., Fu, A., Parak, W.J., Chen, D.J., Majumdar, A., and Alivisatos, A.P. Room-temperature single-nucleotide polymorphism and multiallele DNA detection using fluorescent nanocrystals and microarrays. Anal. Chem. 75, 4766-4772 (2003). 
[24] Micheel, C.M. and Alivisatos, A.P. Correlation Analysis of TEM images of nanocrystal molecules. In preparation.

[25] Loweth, C. I. Organization of nanocrystals using DNA II. Evaluating small molecules as a tool for functional genomics. University of California, Berkeley (2001).

[26] Foos, E., Snow, A.W., Twigg, M.E., and Ancona, M.G. Thiol-terminated di-, tri-, and tetraethylene oxide functionalized gold nanoparticles: a water-soluble, charge-neutral cluster. Chem. Mater. 14, 2401-2408 (2002).

[27] Jhaveri, S.D., Foos, E.E., Lowy, D.A., Chang, E.L., Snow, A.W., and Ancona, M.G. Isolation and characterization of trioxyethylene-encapsulated gold nanoclusters functionalized with a single DNA strand. Nano Lett. 4, 737-740 (2004).

[28] Kanaras, A.G., Wang, Z., Bates, A.D., Cosstick, R., and Brust, M. Towards multistep nanostructure synthesis: programmed enzymatic self-assembly of DNA/gold systems. Angew. Chem. Int. Ed. 42, 191-194 (2003).

[29] Schroedter, A., and Weller, H. Ligand design and bioconjugation of colloidal gold nanoparticles. Angew. Chem. Int. Ed. 41, 3218-3221 (2002). 
[30] Ishii, T., Otsuka, H., Kataoka, K. and Nagasaki, Y. Preparation of functionally PEGylated gold nanoparticles with narrow distribution through autoreduction of auric cation by alpha-biotinyl-PEG-block-[poly $(2-\mathrm{N}, \mathrm{N}-$ dimethylamino)ethyl methacrylate]. Langmuir 20, 561-564 (2004).

[31] Paulini, R., Frankamp, B.L., and Rotello, V.M. Effects of branched ligands on the structure and stability of monolayers on gold nanoparticles. Langmuir 18, 2368-2373 (2002).

[32] Li, Z., Jin, R.C., Mirkin, C.A., and Letsinger, R.L. Multiple thiol-anchor capped DNA-gold nanoparticle conjugates. Nuc. Acids Res. 30, 1558-1562 (2002).

[33] Li, H. and Rothberg, L.J. Label-Free Colorimetric Detection of Specific Sequences in Genomic DNA Amplified by the Polymerase Chain Reaction. J. Am. Chem. Soc. 126, 10958-10961 (2004).

[34] Storhoff, J.J., Elghanian, R., Mirkin, C.A., and Letsinger, R.L. Sequencedependent stability of DNA-modified gold nanoparticles. Langmuir 18, 6666$6670(2002)$. 
[35] Sandstrom, P., Boncheva, M., and Akerman, B. Nonspecific and thiolspecific binding of DNA to gold nanoparticles. Langmuir 19, 7537-7543 (2003).

[36] Otsuka, H., Nagasaki, Y., and Kataoka, K. PEGylated nanoparticles for biological and pharmaceutical applications. Adv. Drug Delivery Rev. 55, 403-419 (2003).

[37] Wang, Y.A., Li, J.J., Chen, H.Y., and Peng, X.G. Stabilization of inorganic nanocrystals by organic dendrons. J. Am. Chem. Soc. 124, 2293-2298 (2002).

[38] Hainfeld, J.F. and Powell, R.D. New frontiers in gold labeling. J. Histochem. Cytochem. 48, 471-480 (2000).

[39] Hainfeld, J.F., and Furuya, F.R. A 1.4-nm gold cluster covalently attached to antibodies improves immunolabeling. J. Histochem. Cytochem. 40, 177-184 (1992).

[40] Powell, R. E-mail to the author. 11 June 2004.

[41] Handley, D.A. in Colloidal Gold: Principles, Methods, and Applications (ed Hayat, M.A.) 1, 13-33 (Academic, New York, 1989). 
[42] de la Torre, B.G., Morales, J.C., Avino, A., lacopino, D., Ongaro, A., Fitzmaurice, D., Murphy, D., Doyle, H., Redmond, G., and Eritja, R. Synthesis of oligonucleotides carrying anchoring groups and their use in the preparation of oligonucleotide-gold conjugates. Helv. Chim. Acta 85, 2594-2607 (2002).

[43] Xiao, S.J., Liu, F.R., Rosen, A.E., Hainfeld, J.F., Seeman, N.C., MusierForsyth, K., and Kiehl, R.A. Selfassembly of metallic nanoparticle arrays by DNA scaffolding. J. Nanopart. Res. 4, 313-317 (2002).

[44] Li, H.Y., Park, S.H., Reif, J.H., LaBean, T.H., and Yan, H. DNA-templated self-assembly of protein and nanoparticle linear arrays. J. Am. Chem. Soc. 126, 418-419 (2004).

[45] Lee, D., Lee, S., Kim, H., and Jang, D.J. Circles of DNA-linked gold nanoparticle strands. Eur. Physical J. D 24, 303-306 (2003).

[46] Tsai, C.-Y., Shiau, A.-L., Cheng, P.-C., Shieh, D.-B., Chen, D.-H., Chou, C.H., Yeh, C.-S., and Wu, C.-L. A biological strategy for fabrication of Au/EGFP nanoparticle conjugates retaining bioactivity. Nano Letters 4, 1209-1212 (2004).

[47] lacopino, D., Ongaro, A., Nagle, L., Eritja, R. and Fitzmaurice, D. Imaging the DNA and nanoparticle components of a self-assembled nanoscale architecture. Nanotech. 14, 447-452 (2003). 
[48] Niemeyer, C.M., Adler, M., Gao, S. and Chi, L.F. Supramolecular nanocircles consisting of streptavidin and DNA. Angew. Chem. Int. Ed. 39, 30553059 (2000).

[49] Yan, H., LaBean, T.H., Feng, L.P. and Reif, J.H. Directed nucleation assembly of DNA tile complexes for barcode-patterned lattices. PNAS 100, 8103-8108 (2003).

[50] Liu, Y.P., Meyer-Zaika, W., Franzka, S., Schmid, G., Tsoli, M. and Kuhn, H. Gold-cluster degradation by the transition of B-DNA into A-DNA and the formation of nanowires. Angew. Chem. Int. Ed. 42, 2853-2857 (2003).

[51] Yun, C.S., Khitrov, G.A., Vergona, D.E., Reich, N.O., and Strouse, G.F. Enzymatic manipulation of DNA-nanomaterial constructs. J. Am. Chem. Soc. $124,7644-7655$ (2002).

[52] Ulanovsky, L., Drouin, G., and Gilbert, W. DNA trapping electrophoresis. Nature 343, 190-192 (1990).

[53] Desruisseaux, C., Slater, G.W., and Drouin, G. On using DNA-trapping electrophoresis to increase the resolution of DNA sequencing gels. Macromolecules 31, 6499-6505 (1998). 
[54] Desruisseaux, C., Slater, G.W., and Kist, T.B.L. Trapping electrophoresis and ratchets: a theoretical study for DNA-protein complexes. Biophysical J. 75, 1228-1236 (1998).

[55] Desruisseaux, C. Long, D., Drouin, G., and Slater, G.W. Electrophoresis of composite molecular objects. 1. relation between friction, charge, and ionic strength in free solution Macromolecules 34, 44-52 (2001).

[56] Slater, G.W., Desruisseaux, C., Hubert, S.J., Mercier, J.-F., Labrie, J., Boileau, J., Tessier, F., and Pepin, M.P. Theory of DNA electrophoresis: a look at some current challenges. Electrophoresis 21, 3873-3887 (2000).

[57] Cobbe, S., Connolly, S., Ryan, D., Nagle, L., Eritja, R. and Fitzmaurice, D. DNA-controlled assembly of protein-modified gold nanocrystals. J. Phys. Chem. B 107, 470-477 (2003).

[58] Connolly, S., Rao, S.N., and Fitzmaurice, D. Characterization of protein aggregated gold nanocrystals. J. Phys. Chem. B 104, 4765-4776 (2000).

[59] Chen, X.D., Jiang, S.G., and Liu, M.H. Progress in DNA-based molecular assemblies. Prog. Chem. 15, 367-373 (2003). 
[60] Penn, S.G., He, L, and Natan, M.J. Nanoparticles for bioanalysis. Curr. Op. Chem. Biol. 7, 609-615 (2003).

[61] Park, S.Y. and Stroud, D. Structure formation, melting, and optical properties of gold/DNA nanocomposites: effects of relaxation time. Phys. Rev. B 68, Art. No. 224201 (2003).

[62] Alivisatos, A.P., Barbara, P.F., Castleman, A.W., Chang, J., Dixon, D.A., Klein, M.L., McLendon, G.L., Miller, J.S., Ratner, M.A., Rossky, P.J., Stupp, S.I., and Thompson, M.E. From molecules to materials: current trends and future directions. Adv. Mat. 10, 1297-1336 (1998).

[63] Brust, M. and Kiely, C.J. Some recent advances in nanostructure preparation from gold and silver particles: a short topical review. Colloids and Surfaces A-Physicochemical and Engineering Aspects 202, 175-186 (2002).

[64] Dubertret, B., Calame, M., and Libchaber, A.J. Single-mismatch detection using gold-quenched fluorescent oligonucleotides. Nature Biotech. 19, 365-370 (2001).

[65] Maxwell, D.J., Taylor, J.R., and Nie, S.M. Self-assembled nanoparticle probes for recognition and detection of biomolecules. J. Am. Chem. Soc. 124, 9606-9612 (2002). 
[66] Hamad-Schifferli, K., Schwartz, J.J., Santos, A.T., Zhang, S.G., and Jacobson, J.M. Remote electronic control of DNA hybridization through inductive coupling to an attached metal nanocrystal antenna. Nature 415, 152-155 (2002).

[67] Hacker, G.W. High performance Nanogold ${ }^{\mathrm{TM}}$-silver in situ hybridization. Eur. J. Histochem. 42, 111-120 (1998).

[68] Hayat, M.A., ed. Colloidal Gold: Principles, Methods, and Applications (Academic, New York, 1989).

[69] Karhanek, M., Kemp, J.T., Pourmand, N., Davis, R.W., and Webb, C.D. Single DNA molecule detection using nanopipettes and nanoparticles. Nano Lett. 5, 403-307 (2005).

[70] Mamedova, N.N., Kotov, N.A., Rogach, A.L, and Studer, J. Albumin-CdTe nanoparticle bioconjugates: preparation, structure and interunit energy transfer with antenna effect. Nano Lett. 1, 281-286 (2001).

[71] Schroedter, A., Weller, H., Eritja, R., Ford, W.E., and Wessels, J.M. Biofunctionalization of silica-coated CdTe and gold nanocrystals. Nano Lett., 2, 1363-1367 (2002). 
[72] Shimizu, K.T., Woo, W.K., Fisher, B.R., Eisler, H.J., and Bawendi, M.G. Surface-enhanced emission from single semiconductor nanocrystals. Phys. Rev. Lett. 89, Art. No. 117401 (2002).

[73] Kulakovich, O., Strekal, N., Yaroshevich, A., Maskevich, S., Gaponenko, S., Nabiev, I., Woggon, U., and Artemyev, M. Enhanced luminescence of CdSe quantum dots on gold colloids. Nano Lett. 2, 1450-1452 (2002).

[74] Gueroui, Z. and Libchaber, A. Single-molecule measurements of goldquenched quantum dots. Phys. Rev. Lett. 93, 166108-1-166108-4 (2004).

[75] Grimau, M.G., lacopino, D., Avino, A., de la Torre, B.G., Ongaro, A., Fitzmaurice, D., Wessels, J., and Eritja, R. Synthesis of branched oligonucleotides as templates for the assembly of nanomaterials. Helv. Chim. Acta 86, 2814-2826 (2003).

[76] Shchepinov, M.S., Mir, K.U., Elder, J.K., Frank-Kamenetskii, M.D., and Southern E.M. Oligonucleotide dendrimers: stable nano-structures. Nuc. Acids. Res. 27, 3035-3041 (1999).

[77] Zheng, M. and Huang, X. Nanoparticles comprising a mixed monolayer for specific bindings with biomolecules. J. Am. Chem. Soc. 126, 12047-12054 (2004). 
[78] Yang, J., Mayer, M., Kreibel, J.K., Garstecki, P., and Whitesides, G.M. Selfassembled aggregates of IgGs as templates for the growth of clusters of gold nanoparticles. Angew. Chem. Int. Ed. 43, 1555-1558 (2004).

[79] Mornet, S., Vekris, A., Bonnet, J., Duguet, E., Grasset, F., Choy, J.H., Portier, J. DNA-magnetite nanocomposite materials. Mat. Lett. 42, 183-188 (2000).

[80] Seidel, R., Ciacchi, L.C., Wegel, M., Pompe, W., and Mertig, M. Synthesis of platinum cluster chains on DNA templates: conditions for a template-controlled cluster growth. J. Phys. Chem. B 108, 10801-10811 (2004).

[81] Kanthimanthi, M., Dhathathreyan, A., and Nair, B.U. Nanosized nickel oxide using bovine serum albumin as template. Mat. Lett. 58, 2914-1917 (2004).

[82] Medalia, O., Heim, M., Guckenberger, R., Sperling, R., and Sperling, J. Gold-tagged RNA-a probe for macromolecular assemblies. J. Struct. Biol. 127, 113-119 (1999).

[83] Huang, Y.F., Huang, C.C., and Chang, H.T. Exploring the activity and specificity of gold nanoparticle-bound trypsin by capillary electrophoresis with laser-induced fluorescence detection. Langmuir 19, 7498-7502 (2003). 
[84] Flierl, A., Jackson, C. Cottrell, B., Murdock, D. Seibel, P. and Wallace, D.C. Targeted delivery of DNA to the mitochondrial compartment via import sequenceconjugated peptide nucleic acid. Mol. Ther. 7, 550-557 (2003).

[85] Alivisatos, P. The use of nanocrystals in biological detection. Nature Biotech. 22, 47-52 (2004).

[86] Chan, W.C.W. and Nie, S. Quantum dot bioconjugates for ultrasensitive nonisotopic detection. Science 281, 2016-2018 (1998).

[87] Storhoff, J.J., Elghanian, R., Mucic, R.C., Mirkin, C.A., and Letsinger, R.L. One-pot colorimetric differentiation of polynucleotides with single base imperfections using gold nanoparticle probes. J. Am. Chem. Soc. 120, 19591964 (1998).

[88] Mucic, R.C., Storhoff, J.J., Mirking, C.A., and Letsinger, R.L. DNA-directed synthesis of binary nanoparticle network materials. J. Am. Chem. Soc. 120, 12674-12675 (1998).

[89] Winfree, E., Liu, F., Wenzler, L.A., and Seeman, N.C. Design and selfassembly of two-dimensional DNA crystal. Nature 394, 539-544 (1998). 
[90] Yurke, B., Turberfield, A.J., Mills, A.P., Simmel, F.C., and Neumann, J.L. A DNA-fuelled molecular machine made of DNA. Nature 406, 605-608 (2000).

[91] Fritz, J., Baller, M.K., Lang, H.P., Rothuizen, H., Vettiger, P., Meyer, E., Güntherodt, H.-J., Gerber, Ch., and Gimzewski, J.K. Translating biomolecular recognition into nanomechanics. Science 288, 316-318 (2000).

[92] Braun, E., Eichen, Y., Sivan, U., and Ben-Yoseph, G. DNA-templated assembly and electrode attachment of a conducting silver wire. Nature 391, 775778 (1998).

[93] Fahlman, R.P. and Sen, D. "Synapsable" DNA double helices: self-selective modules for assembling DNA superstructures. J. Am. Chem. Soc. 121, 1107911085 (1999).

[94] Connolly, S. and Fitzmaurice, D. Programmed assembly of gold nanocrystals in aqueous solution. Adv. Mat. 11, 1202-1205 (1999).

[95] Shenton, W., Davis, S.A., and Mann, S. Directed self-assembly of nanoparticles into macroscopic materials using antibody-antigen recognition. Adv. Mat. 11, 449-452 (1999).

[96] Hermanson, G.T. Bioconjugate Techniques (Academic, New York, 1996). 
[97] Taton, T.A., Mirkin, C.A., and Letsinger, R.L. Scanometric DNA array detection with nanoparticle probes. Science 289, 1757-1760 (2000).

[98] Tinland, B., Pluen, A., Sturm, J., and Weill, G. Persistence length of singlestranded DNA. Macromolecules 30, 5763-5765 (1997).

[99] Noolandi, J. in Advances in Electrophoresis (ed Chrambach, A.) 5, 2-57 (Weinheim, Germany, 1998).

[100] Schaaf, T.G., Knight, G., Shafigullin, M.N., Borkman, R.F., and Whetten, R.L. Isolation and selected properties of a $10.5 \mathrm{kDa}$ gold:glutathione cluster compound. J. Phys. Chem. B 102, 10643-10646 (1998).

[101] Herne, T.M. and Tarlov, M.J. Characterization of DNA probes immobilized on gold surfaces. J. Am. Chem. Soc. 119, 8916-8920 (1997).

[102] Ferguson, K.A. Starch-gel electrophoresis-application to classification of pituitary proteins + polypeptides. Metabolism 13, 985 (1964).

[103] Slater, G.W. and Guo, H.L. An exactly solvable Ogston model of gel electrophoresis. 1 . The role of symmetry and randomness of the gel structure. Electrophoresis 17, 623-632 (1996). 
[104] Tietz, D. and Chrambach, A. Computer-simulation of the variable agarose fiber dimensions on the basis of mobility data derived from gel-electrophoresis and using the Ogston theory. Anal. Biochem. 161, 395-411 (1987).

[105] Parak, W.J., Gerion, D., Zanchet, D., Woerz, A.S., Pellegrino, T., Micheel, C., Williams, S.C., Seitz, M., Bruehl, R.E., Bryant, Z., Bustamante, C., Bertozzi, C.R., and Alivisatos, A.P. Conjugation of DNA to silanized colloidal semiconductor nanocrystalline quantum dots. Chem. Mater. 14, 2113-2119 (2002).

[106] Freitag, S., Le Trong, I., Chilkoti, A., Klumb, L.A., Stayton, P.S., and Stenkamp, R.E. Structural studies of binding site tryptophan mutants in the highaffinity streptavidin-biotin complex. J. Mol. Biol. 279, 211-221 (1998).

[107] Nicewarner Pena, S.R., Raina, S., Goodrich, G.P., Fedoroff, N.V., and Keating, C.D. Hybridization and enzymatic extension of Au nanoparticle-bound oligonucleotides. J. Am. Chem. Soc. 124, 7314-7323 (2002).

[108] Shoneborn, H., Bulle, J., and von Kiedrowski, G. Kinetic monitoring of selfreplicating systems through measurement of fluorescence resonance energy transfer. ChemBioChem 2, 922-927 (2001). 
[109] Luther, A., Brandsch, R. and von Kiedrowski, G. Surface-promoted replication and exponential amplification of DNA analogues. Nature 396, 245248 (1998).

[110] James, K.D. and Ellington, A.D. Surprising fidelity of template-directed chemical ligation of oligonucleotides. Chem. \& Biol. 4, 595-605 (1997).

[111] Zhan, Z.-Y. J. and Lynn, D.G. Chemical amplification through templatedirected synthesis. J. Am. Chem. Soc. 119, 12420-12421 (1997).

[112] Fujimoto, K., Matsuda, S., Takahashi, N., and Saito, I. Template-directed photoreversible ligation of deoxyoligonucleotides via 5-vinyldeoxyuridine. J. Am. Chem. Soc. 122, 5646-5647 (2000).

[113] Xu, Y. and Kool, E.T. Rapid and selective selenium-mediated autoligation of DNA strands. J. Am. Chem. Soc. 122, 9040-9041 (2000).

[114] Albagli, D., Van Atta, R., Cheng, P., Huan, B., and Wood, M.L. Chemical amplification (CHAMP) by a continuous, self-replicating oligonucleotide-based system. J. Am. Chem. Soc. 121, 6954-6955 (1999). 
[115] Zheng, M. Davidson, F., and Huang, X. Ethylene glycol monolayer protected nanoparticles for eliminating nonspecific binding with biological molecules. J. Am. Chem. Soc. 125, 7790-7791 (2003).

[116] Nednoor, P., Capaccio, M., Gavalas, V.G., Meier, M.S., Anthony, J.E., and Bachas, L.G. Hybrid nanoparticles based on organized protein immobilization on fullerenes. Bioconjugate Chem. 15, 12-15 (2004).

[117] Watson, K.J., Park, S.J., Im, J.H., Nguyen, S.T., and Mirkin, C.A. DNAblock copolymer conjugates. J. Am. Chem. Soc. 123, 5592-5593 (2001).

[118] Li, M., Wong, K.K.W., and Mann, S. Organization of inorganic nanoparticles using biotin-streptavidin connectors. Chem. Mater. 11, 23-26 (1999).

[119] Niemeyer, C.M., Adler, M., Gao, S., and Chi, L.F. Nanostructured DNAprotein aggregates consisting of covalent oligonucleotide-streptavidin conjugates. Bioconj. Chem. 12, 364-371 (2001).

[120] Murphy, D., Redmond, G., de la Torre, B.G., and Eritja, R. Hybridization and melting behavior of peptide nucleic acid (PNA) oligonucleotide chimeras conjugated to gold nanoparticles. Helv. Chim. Acta 87, 2727-2734 (2004). 
[121] Shu, D., Moll, W.-D., Deng, Z., Mao, C., and Guo, P. Bottom-up assembly of RNA arrays and superstructures as potential parts in nanotechnology. Nano Lett. 4, 1717-1723 (2004).

[122] Wang, Z.X., Kanaras, A.G., Bates, A.D., Cosstick, R. and Brust, M. Enzymatic DNA processing on gold nanoparticles. J. Mat. Chem. 14, 578-580 (2004).

[123] Mao, C.D., Sun, W.Q., Shen, Z.Y., and Seeman, N.C. A nanomechanical device based on the B-Z transition of DNA. Nature 397, 144-146 (1999).

[124] Fahlmann, R.P., Hsing, M., Sporer-Tuhten, C.S., and Sen, D. Duplex pinching: a structural switch suitable for contractile DNA nanoconstructions. Nano Lett. 3, 1073-1078 (2003).

[125] Fahlman, R.P. and Sen, D. "Synapsable" DNA double helices: selfselective modules for assembling DNA superstructures. J. Am. Chem. Soc. 121, 11079-11085 (1999).

[126] Chen, Y. and Mao, C. Reprogramming DNA-directed reactions on the basis of a DNA conformational change. J. Am. Chem. Soc. 126, 13240-13241 (2004). 
[127] Dujardin, E., Peet, C., Stubbs, G., Culver, J.N., and Mann, S. Organization of metallic nanoparticles using tobacco mosaic virus templates. Nano Lett. 3 , 413-417 (2003).

[128] Liu, J. and Lu, Y. Accelerated color change of gold nanoparticles assembled by DNAzymes for simple and fast colorimetric $\mathrm{Pb}^{2+}$ detection. J. Am. Chem. Soc. 126, 12298-12305 (2004).

[129] Liu, J. and Lu, Y. Optimization of a $\mathrm{Pb}^{2+}$-directed gold nanoparticle/DNAzyme assembly and its application as a colorimetric biosensor for $\mathrm{Pb}^{2+}$. Chem. Mater. 16, 3231-3238 (2004).

[130] Eichen, Y., Braun, E., Silva, U. and Ben-Yoseph, G. Self-assembly of nanoelectric components and circuits using biological templates. Acta Polymerica 49, 663-670 (1998).

[131] Keren, K., Berman, R.S., Buchstab, E., Sivan, U., and Braun, E. DNAtemplated carbon nanotube field-effect transistor. Science 302, 1380-1382 (2003).

[132] Cassell, A.M., Scrivens, W.A., and Tour, J.M. Assembly of DNA/fullerene hybrid materials. Angew. Chem. Int. Ed. 37, 1528-1531 (1998). 
[133] Maeda, Y., Nakamura, Uchimura, K., Matsumoto, T., Tabata, H. and Kawai, T. Controlled conjugation of nanoparticles with single stranded DNA. J. Vac. Sci. Technol. B 17, 494-496 (1999).

[134] Maeda, Y. Tabata, H., and Kawai, T. Two-dimensional assembly of gold nanoparticles with a DNA network template. App. Phys. Lett. 79, 1181-1183 (2001).

[135] Woehrle, G.H., Warner, M.G., and Hutchison, J.E. Molecular-level control of feature separation in one-dimensional nanostructure assemblies formed by biomolecular nanolithography. Langmuir 20, 5982-5988 (2004).

[136] Nelson, D.R. Toward a tetravalent chemistry of colloids. Nano Lett. 2, $1125-1129$ (2002).

[137] Michalet, X. Stretching single-stranded DNA on a surface. Nano Lett. 1, 341-343 (2001).

[138] Woolley, A.T. and Kelly, R.T. Deposition and characterization of extended single-stranded DNA molecules on surfaces. Nano Lett. 1, 345-348 (2001). 
[139] Diez, S., Reuther, C., Dinu, C., Seidel, R., Mertig, M., Pompe, W., and Howard, J. Stretching and transporting DNA molecules using motor proteins. Nano Lett. 3, 1251-1254 (2003).

[140] Reif, J.H. The emerging discipline of biomolecular computation in the US. New Gen. Comp. 20, 217-236 (2002).

[141] Brown, K.R., Lidar, D.A., and Whaley, K.B. Quantum computing with quantum dots on quantum linear supports. Phys. Rev. A 65, Art. No. 012307 (2002).

[142] Shin, J.-S. and Pierce, N.A. Rewritable memory by controllable nanopatterning of DNA. Nano Lett. 4, 905-909 (2004). 